\title{
MODULES FOR EXPERIMENTS IN STELLAR ASTROPHYSICS (MESA): PLANETS, OSCILLATIONS, ROTATION, AND MASSIVE STARS
}

\author{
Bill Paxton $^{1}$, Matteo Cantiello $^{1}$, Phil $_{\text {Arras }}^{2}$, Lars Bildsten $^{1,3}$, Edward F. Brown ${ }^{4}$, Aaron Dotter ${ }^{5}$, \\ Christopher Mankovich ${ }^{3}$, M. H. Montgomery ${ }^{6}$, Dennis STello $^{7}$, F. X. Timmes 8 , and Richard Townsend ${ }^{9}$ \\ ${ }^{1}$ Kavli Institute for Theoretical Physics, University of California, Santa Barbara, CA 93106, USA; matteo@ kitp.ucsb.edu \\ ${ }^{2}$ Department of Astronomy, University of Virginia, P.O. Box 400325, Charlottesville, VA 22904-4325, USA \\ ${ }^{3}$ Department of Physics, University of California, Santa Barbara, CA 93106, USA \\ ${ }^{4}$ Department of Physics and Astronomy, National Superconducting Cyclotron Laboratory, and Joint Institute for Nuclear Astrophysics, \\ Michigan State University, East Lansing, MI 48864, USA \\ ${ }^{5}$ Research School of Astronomy and Astrophysics, The Australian National University, Weston, ACT 2611, Australia \\ ${ }^{6}$ Department of Astronomy and McDonald Observatory, University of Texas, Austin, TX 78712, USA \\ ${ }^{7}$ Sydney Institute for Astronomy (SIfA), School of Physics, University of Sydney, NSW 2006, Australia \\ ${ }^{8}$ School of Earth and Space Exploration, Arizona State University, Tempe, AZ 85287, USA \\ ${ }^{9}$ Department of Astronomy, University of Wisconsin-Madison, Madison, WI 53706, USA \\ Received 2012 December 20; accepted 2013 June 10; published 2013 August 28
}

\begin{abstract}
We substantially update the capabilities of the open source software package Modules for Experiments in Stellar Astrophysics (MESA), and its one-dimensional stellar evolution module, MESAstar. Improvements in MESAstar's ability to model the evolution of giant planets now extends its applicability down to masses as low as one-tenth that of Jupiter. The dramatic improvement in asteroseismology enabled by the space-based Kepler and CoRoT missions motivates our full coupling of the ADIPLS adiabatic pulsation code with MESAstar. This also motivates a numerical recasting of the Ledoux criterion that is more easily implemented when many nuclei are present at non-negligible abundances. This impacts the way in which MESAstar calculates semi-convective and thermohaline mixing. We exhibit the evolution of 3-8 $M_{\odot}$ stars through the end of core He burning, the onset of He thermal pulses, and arrival on the white dwarf cooling sequence. We implement diffusion of angular momentum and chemical abundances that enable calculations of rotating-star models, which we compare thoroughly with earlier work. We introduce a new treatment of radiation-dominated envelopes that allows the uninterrupted evolution of massive stars to core collapse. This enables the generation of new sets of supernovae, long gamma-ray burst, and pair-instability progenitor models. We substantially modify the way in which MESAstar solves the fully coupled stellar structure and composition equations, and we show how this has improved the scaling of MESA's calculational speed on multi-core processors. Updates to the modules for equation of state, opacity, nuclear reaction rates, and atmospheric boundary conditions are also provided. We describe the MESA Software Development Kit that packages all the required components needed to form a unified, maintained, and well-validated build environment for MESA. We also highlight a few tools developed by the community for rapid visualization of MESAstar results.
\end{abstract}

Key words: asteroseismology - methods: numerical - planets and satellites: physical evolution - stars: evolution - stars: massive - stars: rotation Online-only material: color figures

\section{CONTENTS}

1. INTRODUCTION

2. GIANT PLANETS AND LOW-MASS STARS

2.1. Construction of Starting Models

2.2. Evolutionary Calculations

2.3. Implementation of Inert Cores

2.4. Irradiation

2.5. Low-mass Main Sequence Stars

3. ASTEROSEISMOLOGY

3.1. The Solar Sound Speed Profile

3.2. New Asteroseismic Capabilities in MESA

3.3. The Effect of Composition Gradients on the Brunt-Väisälä Frequency

4. MIXING MECHANISMS INVOLVING COMPOSITION GRADIENTS

4.1. Semiconvection

4.2. Thermohaline Mixing

4.3. Impact of Mixing on Convective Core Hydrogen and Helium Burning

5. EVOLUTION BEYOND THE MAIN

SEQUENCE AND WHITE DWARFS 
5.1. Code Comparisons during Helium Core Burning 13

5.2. Making and Cooling White Dwarfs 14

5.3. Compressional Heating and Accretion 17

6. ROTATION 18

6.1. Implementation of Shellular Rotation 18

$\begin{array}{ll}\text { 6.1.1. Stellar Structure } & 18\end{array}$

6.1.2. Mixing and Angular Momentum Transport 19

6.2. Internal Magnetic Fields 19

6.3. Surface Magnetic Fields 19

6.4. Rotationally Enhanced Mass Loss 20

\begin{tabular}{ll} 
6.5. & Initial Models \\
\hline 6.6. & 20
\end{tabular}

6.6. Test Cases: $15 M_{\odot}$ and $25 M_{\odot} \quad 20$

6.7. Rapidly Rotating Massive Stars 20

7. MASSIVE STELLAR EVOLUTION 23

7.1. Evolution of Massive Stars with MESA

7.2. Treatment of Superadiabatic Convection in Radiation-dominated Regions 27

$\begin{array}{lll}\text { 7.3. Core-collapse Progenitor Models } & 28\end{array}$

7.4. Radial Instability of Red Supergiants 30

8. SUMMARY AND CONCLUSIONS 30

APPENDIX A UPDATES TO INPUT PHYSICS MODULES

A.1. Atomic and Nuclear Data 31

$\begin{array}{ll}\text { A.2. Equation of State } & 32\end{array}$

\begin{tabular}{lr} 
A.3. Opacities & 32 \\
\hline
\end{tabular}

A.4. Nuclear Reactions $\quad 32$

A.5. Atmosphere Boundary Conditions 33

APPENDIX B NUTS AND BOLTS 33

B.1. Evolve a Step $\quad 33$

B.2. Solving the Coupled Structure, Burn, and Mix Equations

B.3. Time Step Controls $\quad 36$

B.4. Mesh Controls $\quad 36$

B.5. Mass Adjustment $\quad 37$

B.6. Evolving the Angular Velocity 37

$\begin{array}{ll}\text { B.7. Free Parameters } & 38\end{array}$

$\begin{array}{ll}\text { B.7.1. Convection } & 38\end{array}$

$\begin{array}{ll}\text { B.7.2. Overshooting } & 38\end{array}$

$\begin{array}{ll}\text { B.7.3. Semiconvection } & 38\end{array}$

$\begin{array}{ll}\text { B.7.4. Thermohaline Mixing } & 39\end{array}$

B.8. Nuclear Reactions $\quad 39$

B.9. Multicore Performance $\quad 39$

B.10. Visualization 40

B.11. Operating System and Compiler Considerations 40

APPENDIX C THE MESA SOFTWARE DEVELOPMENT KIT 40

REFERENCES

\section{INTRODUCTION}

As the most commonly observed objects, stars remain at the forefront of astrophysical research. Advances in optical detector technology, computer processing power, and data storage capability have enabled new sky surveys (e.g., the Sloan Digital Sky Survey; York et al. 2000); triggered many new optical transient surveys, such as the Palomar Transient Factory (Law et al. 2009) and Pan-STARRS1 (Kaiser et al. 2010); and allowed for space missions (e.g., Kepler; Koch et al. 2010) that continuously monitor more than 100,000 stars. The stellar discoveries from these surveys include revelations about rare stars, unusual explosive outcomes, and remarkably complex binaries. The immediate future holds tremendous promise, as both the space-based survey Gaia (de Bruijne 2012; Liu et al. 2012) and the ground-based Large Synoptic Survey Telescope (Ivezic et al. 2008) come to fruition.
These developments have created a new demand for a reliable and publicly available research and education tool in computational stellar astrophysics. We introduced the open source community tool Modules for Experiments in Stellar Astrophysics (MESA; Paxton et al. 2010, hereafter Paper I) to meet these new demands. This first "instrument" paper described the design, implementation, and realm of validity of MESA modules for numerics, microphysics, and macrophysics, and introduced the stellar evolution module, MESAstar. We presented a multitude of tests and code comparisons that served as our initial verification and demonstrated MESAstar's initial capabilities. Since Paper I, MESA has attracted over 500 registered users, witnessed over 5000 downloads from http://mesa.sourceforge.net/, started an annual Summer School program, and provided a portal (http://mesastar.org) for the community to openly share knowledge (e.g., the specific settings for a published MESAstar run), codes, and publications. 
This paper describes the major new MESA capabilities for modeling giant planets, asteroseismology, and the treatment of rotation and evolution of massive stars. We also describe numerous advances since Paper I. These include the incorporation of composition gradients in the determination of convective mixing and additional verification for evolution of intermediate mass stars and the white dwarfs (WDs) they create.

Our improvements to MESAstar for gas giant planets were motivated by the dramatic growth in this field. Over 800 exoplanets have been confirmed, and their study has prompted enormous progress in our understanding of the formation and migration of giant planets, and of the importance of factors such as stellar mass (Laughlin et al. 2004; Alibert et al. 2011; Boss 2011), composition (Fischer \& Valenti 2005; Young et al. 2012), and binarity (Patience et al. 2002; Mugrauer \& Neuhäuser 2009; Roell et al. 2012). Puzzles remain, though, both in our solar system and in the studies of the plethora of these newly discovered exoplanets, including the characteristics of the planet-hosting stars and the interiors, atmospheres, surface gravities, temperatures, and compositions of the planets (e.g., Udry \& Santos 2007; Seager \& Deming 2010). Many of these variations can now be numerically explored, as can the incorporation of an inert core in an otherwise regular gas giant and the impact of irradiation.

The ability to infer stellar properties (e.g., mass, radius, internal state, and rotation) from measurements of the radial and non-radial oscillation modes has been dramatically improved by two space-based optical telescopes (Convection Rotation and Planetary Transits, CoRoT, Baglin et al. 2009; Kepler, Borucki et al. 2009). The high cadences and precision (often better than 10 parts per million) reveal and accurately measure multitudes of oscillation frequencies for over 10,000 stars, substantially raising the need for accurate and efficient computations of stellar mode frequencies and the resulting eigenfunctions. The intrinsic flexibility of MESAstar allows for the exploration of model-space required to precisely infer stellar properties from the observed frequencies.

An important new addition to MESA is the incorporation of stellar rotation and magnetic fields in radiative regions. As stars are not solid bodies, they undergo radial differential rotation (Thompson et al. 2003; Balbus et al. 2012) and also rotate at different angular velocities at different latitudes (Ruediger et al. 1998; Bonanno et al. 2007; Küker et al. 2011). These rotational shears have a significant impact on the evolution of the stellar magnetic field. Despite the resulting three-dimensional (3D) nature of magnetism and rotation, the stellar evolution community has come a long way in understanding stars with 1D simulations (Meynet \& Maeder 1997; Langer et al. 1999; Maeder \& Meynet 2000b; Heger \& Langer 2000; Hirschi et al. 2004; Cantiello \& Langer 2010), thus motivating our need to fully incorporate rotation within MESA. The new flexibility in angular momentum transport mechanisms allows for numerical exploration of alternate rotational outcomes should the observations (e.g., asteroseismology) require it.

The paper is outlined as follows. Section 2 describes the new capability of MESA to evolve models of giant planets, while Section 3 discusses the new asteroseismology capabilities. The MESA implementation of composition gradients in stellar interiors and their impact on convective mixing is described in Section 4. The status of the evolution of intermediate mass stars and the MESAstar construction and evolution of WDs is described in Section 5. The new capabilities for evolving rotating stars is described in Section 6. The onset of near
Eddington luminosities and radiation pressure dominance in the envelopes of evolving massive stars has been a challenge for many stellar evolution codes ever since the realization of the iron opacity bump at $\log T \approx 5.3$ (Iglesias et al. 1992). We discuss in Section 7 the resulting improvements for evolving massive stars. This allows for the uninterrupted evolution of rotating massive stars to the onset of core collapse. We conclude in Section 8 by highlighting where additional improvements to MESA are likely to occur in the near future. Appendix A describes the many improvements to the physics modules since Paper I, Appendix B presents "nuts and bolts" information on the primary components of evolution calculations, and Appendix C presents the MESA Software Development Kit (SDK). All of our symbols are defined in Table 1 . We denote components of MESA, such as modules and routines, in Courier font, e.g., evolve_star.

\section{GIANT PLANETS AND LOW-MASS STARS}

Evolutionary models of giant planets and low-mass stars differ from their higher-mass stellar counterparts in both the microphysics needed to describe the interior and the role of stellar irradiation in the outer boundary condition. For masses $M \lesssim 84 M_{\mathrm{J}}$, hydrogen burning is insufficient to prevent cooling and contraction. Deuterium burning can briefly slow the cooling for $M \gtrsim$ $13 M_{\mathrm{J}}$, where $M_{\mathrm{J}}=9.54 \times 10^{-4} M_{\odot}$ is Jupiter's mass, but has a negligible influence on the cooling for smaller masses. Hence nuclear burning can be ignored in the planetary mass regime.

For hydrogen-helium rich objects with $M \gg M_{\mathrm{J}}$, an ideal gas equation of state (EOS), with arbitrary degeneracy, is a good approximation while for $M \lesssim M_{\mathrm{J}}$ particle interactions play an important role. Specifically, pressure ionization of hydrogen at $\rho \simeq 1 \mathrm{~g} \mathrm{~cm}^{-3}$ and $T \simeq 10^{4} \mathrm{~K}$ causes a sudden change from a $\mathrm{H}_{2}$-dominated phase to an ionized phase. MESA employs the Saumon et al. (1995) EOS (SCVH EOS), smoothly interpolated from the low to high pressure phase, for this complicated region of parameter space where thermal, Fermi, and electrostatic energies may all be comparable. The SCVH EOS includes pressure ionization of hydrogen, but not helium. The temperature range covered by the tables is $2.10<\log T(\mathrm{~K})<7.06$, and the pressure ranges from $\log P\left(\right.$ dyne $\left.\mathrm{cm}^{-2}\right)=4$ to a maximum value 19 dependent on the temperature. Smooth interpolation to other EOS occurs near the SCVH boundaries (for more details see Paper I). At the low temperatures in planetary atmospheres, abundant species such as CNO atoms will be in molecular form, and may condense into clouds. MESA does not follow the transition from atomic to molecular form for these species in the EOS - they are currently included by increasing the helium abundance from $Y$ to $Y+Z$ when calling the SCVH EOS. MESA does, however, include the effect of molecules in the Rosseland opacities. Currently, the Ferguson et al. (2005) and Freedman et al. (2008) tables, which include the opacity from molecules, but ignore condensates, are available.

Lastly, for planets in close-in orbits about their parent star, the external irradiation flux may be orders of magnitude larger than the cooling flux from the planet's interior. This may dramatically increase the surface temperature and affect the outer boundary condition. MESA now implements several options for this surface heating, including the flexibility to include usersupplied prescriptions.

In the following subsections, we discuss a new MESA module that creates initial models in the planetary mass range $M \simeq$ $0.1-10 M_{\mathrm{J}}$, and present a suite of evolutionary calculations. We discuss how surface irradiation may be included, as well as 
Table 1

Variable Index

\begin{tabular}{|c|c|c|}
\hline Name & Description & First Appears \\
\hline$A$ & Atomic mass number & A. 1 \\
\hline$\Delta_{i}$ & Mass excess of the $i$ th isotope & A. 1 \\
\hline$\eta$ & Wind mass loss coefficient & 5.2 \\
\hline$F_{\star}$ & Day-side flux incident on an irradiated planet & 2.4 \\
\hline$\Gamma$ & Coulomb coupling parameter & 5.2 \\
\hline$i$ & Specific moment of inertia & B.6 \\
\hline$\kappa$ & Opacity & 2.1 \\
\hline$L$ & Stellar luminosity & 3.2 \\
\hline$m$ & Lagrangian mass coordinate & 2.1 \\
\hline$M$ & Stellar mass & 2.1 \\
\hline$N$ & Brunt-Väisälä frequency & 3.3 \\
\hline$n_{i}$ & Number density of the $i$ th isotope & A. 1 \\
\hline$v$ & Turbulent viscosity & B.6 \\
\hline$r$ & Radial coordinate & 2.4 \\
\hline$R$ & Total stellar radius & 2.1 \\
\hline$\rho$ & Baryon mass density & A. 1 \\
\hline$S$ & Specific entropy & 2.1 \\
\hline$\Sigma$ & Mass column & 2.4 \\
\hline$\Sigma_{\star}$ & Depth for heating from irradiation & 2.4 \\
\hline$\tau$ & Optical depth & 5.2 \\
\hline$w_{c}$ & Magnitude of changes during a time step & B. 3 \\
\hline$w_{t}$ & Target value for $w_{c}$ & B. 3 \\
\hline$W$ & Atomic weight & A.1 \\
\hline$X$ & $\mathrm{H}$ mass fraction & 3.2 \\
\hline$X_{i}$ & Baryon mass fraction of the $i$ th isotope & 3.3 \\
\hline$Y$ & He mass fraction & 2 \\
\hline$Y_{e}$ & Electrons per baryon $(\bar{Z} / \bar{A})$ & A. 1 \\
\hline$Y_{i}$ & Abundance of the $i$ th isotope & A.1 \\
\hline$Z$ & Metallicity & 2 \\
\hline$Z$ & Atomic number & A. 1 \\
\hline$\alpha_{\mathrm{MLT}}$ & Mixing length parameter & 2.2 \\
\hline$\alpha_{\mathrm{sc}}$ & Semiconvection efficiency parameter & 4.1 \\
\hline$\alpha_{\mathrm{th}}$ & Thermohaline efficiency parameter & 4.2 \\
\hline$\alpha_{\nabla}$ & Smoothing parameter for MLT++ & 7.2 \\
\hline$\widetilde{\alpha_{\nabla}}$ & MLT++ parameter used in construction of $\alpha_{\nabla}$ & 7.2 \\
\hline$\beta_{\min }$ & $\min \left(P / P_{\text {gas }}\right)$ & 7.2 \\
\hline$\chi_{\rho}$ & $(\partial \ln P / \partial \ln \rho)_{T}$ & 3.3 \\
\hline$\chi_{T}$ & $(\partial \ln P / \partial \ln T)_{\rho}$ & 3.3 \\
\hline$C_{P}$ & Specific heat at constant pressure & 4.1 \\
\hline$c_{\mathrm{S}}$ & Adiabatic sound speed & 3 \\
\hline$\Delta v$ & Large frequency separation of pulsation modes & 3.2 \\
\hline$D_{\mathrm{ov}}$ & Overshoot diffusion coefficient & 3.3 \\
\hline$D_{\text {th }}$ & Thermohaline diffusion coefficient & 4.2 \\
\hline$E_{\mathrm{F}, \mathrm{c}}$ & Fermi energy at center & 2.2 \\
\hline$\epsilon_{\text {grav }}$ & Gravitational heating rate & 5.2 \\
\hline$\epsilon_{\text {nuc }}$ & Nuclear heating rate & A. 4 \\
\hline$\epsilon_{v}$ & Neutrino loss rate & 6.1 \\
\hline$F_{\text {conv }}$ & Convective flux & 7.1 \\
\hline$f_{\mathrm{OV}}$ & Convective overshoot parameter & 3.2 \\
\hline$F_{\text {rad }}$ & Radiative flux & 7.1 \\
\hline$f_{\nabla}$ & Reduction factor for $\delta_{\nabla}$ & 7.2 \\
\hline$\Gamma_{1}$ & $(\partial \ln P / \partial \ln \rho)_{S}$ & 3 \\
\hline$\kappa_{\text {th }}$ & Opacity for thermal radiation orig. in planet & 2.4 \\
\hline$\kappa_{\mathrm{V}}$ & Opacity for irradiation from star & 2.4 \\
\hline$k_{\mathrm{B}}$ & Boltzmann constant & 2.2 \\
\hline$L_{\mathrm{acc}}$ & Accretion luminosity & 5.3 \\
\hline$\lambda_{\max }$ & $\max \left(L_{\mathrm{rad}} / L_{\mathrm{Edd}}\right)$ & 7.2 \\
\hline$L_{\mathrm{c}}$ & Core luminosity & 2.3 \\
\hline$L_{\text {Edd }}$ & Eddington luminosity & 6.4 \\
\hline $\log g$ & $\log$ surface gravity & A. 5 \\
\hline$L_{\text {onset }}$ & Luminosity at which the onset of convection occurs & 7.1 \\
\hline$L_{\mathrm{rad}}$ & Radiative luminosity & 7.1 \\
\hline$L_{\text {inv }}$ & Luminosity at which a density inversion occurs & 7.1 \\
\hline$m_{\mathrm{u}}$ & Atomic mass unit & A. 1 \\
\hline$M_{\mathrm{c}}$ & Core mass & 2.3 \\
\hline$\dot{M}$ & Mass-loss rate & 5.3 \\
\hline
\end{tabular}

Table 1

(Continued)

\begin{tabular}{|c|c|c|}
\hline Name & Description & First Appears \\
\hline$M_{\mathrm{m}}$ & Modeled mass & B. 4 \\
\hline$N_{\mathrm{A}}$ & Avogadro number & 2.2 \\
\hline$\nabla_{\mathrm{ad}}$ & Adiabatic temperature gradient & 3.3 \\
\hline$\nabla_{\mathrm{L}}$ & Ledoux criterion & 4.1 \\
\hline$\nabla_{\mathrm{rad}}$ & Radiative temperature gradient & 3.3 \\
\hline$\nabla_{T}$ & Actual temperature gradient & 3.3 \\
\hline$n_{\mathrm{B}}$ & Baryon density & A.1 \\
\hline$v_{\max }$ & Frequency of maximum power & 3.2 \\
\hline$\Omega$ & Surface angular velocity & 6.4 \\
\hline$\omega$ & Angular velocity & 6.1 \\
\hline$\Omega_{\text {crit }}$ & Surface critical angular velocity & 6.4 \\
\hline$P_{\mathrm{c}}$ & Central pressure & 2.1 \\
\hline$P_{\text {gas }}$ & Gas pressure & 5.3 \\
\hline$P_{\text {rad }}$ & Radiation pressure & 5.3 \\
\hline$R_{\mathrm{c}}$ & Core radius & 2.3 \\
\hline$\rho_{\mathrm{c}}$ & Central density & 2.1 \\
\hline$\lambda_{P}$ & Pressure scale height & 3.3 \\
\hline$\sigma_{\mathrm{SB}}$ & Stefan-Boltzmann constant & 2.1 \\
\hline$S_{\ell}$ & Lamb frequency & 3 \\
\hline$\delta_{\nabla}$ & Superadiabaticity, $\nabla_{T}-\nabla_{\mathrm{ad}}$ & 7.2 \\
\hline$\delta_{\nabla, \text { thresh }}$ & Controls when MLT++ is applied & 7.2 \\
\hline$T_{\mathrm{c}}$ & Central temperature & 2.1 \\
\hline$T_{\text {eff }}$ & Effective temperature & 2.1 \\
\hline$\delta t$ & Numerical time step & 5.3 \\
\hline$\tau_{\mathrm{KH}}$ & Thermal (Kelvin-Helmholtz) timescale & 6.4 \\
\hline$v_{\text {eq }}$ & Equatorial velocity & 6 \\
\hline
\end{tabular}

an inert core at the center of the planet. We also show what MESAstar yields for the mass-radius relation for sub-solar mass stars in Section 2.5.

\subsection{Construction of Starting Models}

For stellar mass objects, the pre_ms_model routine constructs pre-main-sequence (PMS) models assuming $L(r) \propto m$, where $L(r)$ is the luminosity at radius $r$, by iterating on the starting conditions at the center to find a model with a given $M$ and central temperature $T_{\mathrm{c}}$. This PMS routine works well for $M \gtrsim 0.03 M_{\odot}$, but lower masses may not converge when the guess for central density $\rho_{\mathrm{c}}$ and luminosity are not close enough to the (unknown) true values. As a result, it is difficult and time consuming to create models with $M<0.03 M_{\odot}$ using the same routine for giant planets as for stars.

A new routine called create_initial_model builds a model of given $M$ and radius $R$ using an adiabatic temperature profile. Given the central pressure $P_{\mathrm{c}}$ and specific entropy $S$, the equation of hydrostatic balance is integrated outward, and the temperature at each step determined from the EOS using $T=T(P, S)$. The values of $P_{\mathrm{c}}$ and $S$ are iterated to attain the desired $M$ and $R$. The luminosity profile is then derived treating $S$ as constant in space for the fully convective planet (e.g., Ushomirsky et al. 1998), so

$$
\int_{0}^{m} d m^{\prime} T\left(m^{\prime}\right) \frac{d S}{d t} \simeq \frac{d S}{d t} \int_{0}^{m} d m^{\prime} T\left(m^{\prime}\right)=-L(m)
$$

The luminosity at the surface, $L(M)$, is estimated using the radius $R$ and temperature $T_{\text {eff }}$ at the $\tau=\kappa P / g=2 / 3$ point as $L(M)=4 \pi R^{2} \sigma_{\mathrm{SB}} T_{\mathrm{eff}}^{4}$. Given $L(M)$, the luminosity at interior 


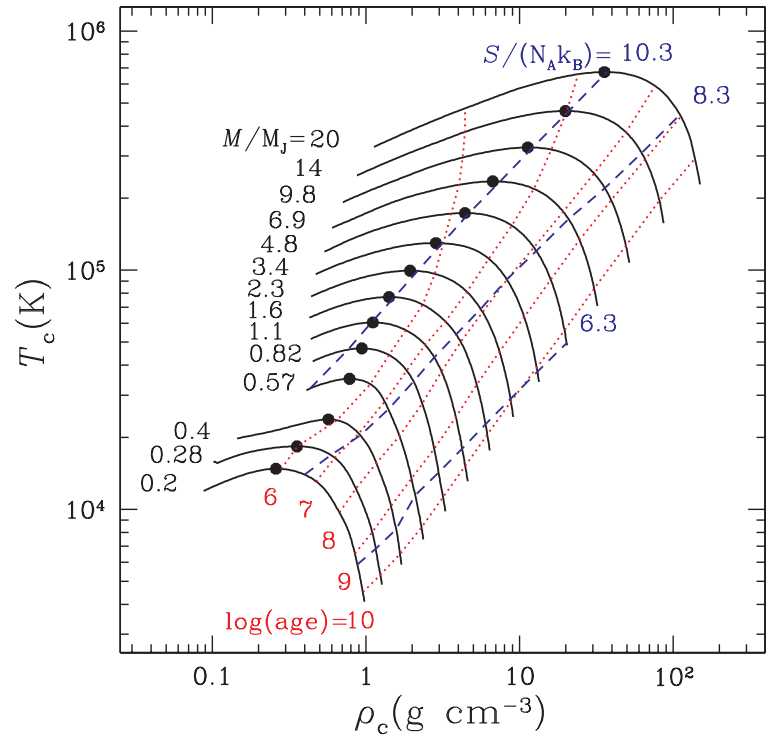

Figure 1. Solid black lines show $T_{\mathrm{c}}$ vs. $\rho_{\mathrm{c}}$ during the evolution. Each line is labeled on the left by the mass in units of $M_{\mathrm{J}}$. The dotted red lines show constant values of $\log (\mathrm{age}[\mathrm{yr}])$, labeled at the base of each line. The blue dashed lines show fixed values of $S /\left(N_{\mathrm{A}} k_{\mathrm{B}}\right)$, labeled at the top of each line. The large black dots show the position of maximum $T_{\mathrm{c}}$ along the evolutionary track.

(A color version of this figure is available in the online journal.)

points is found using

$$
L(m)=L(M)\left(\frac{\int_{0}^{m} d m^{\prime} T\left(m^{\prime}\right)}{\int_{0}^{M} d m^{\prime} T\left(m^{\prime}\right)}\right) .
$$

This procedure works well for $M$ down to $\sim 0.1 M_{\mathrm{J}}$ and over a range of initial radii, allowing the user to choose either a $\sim 1 R_{\mathrm{J}}$ radius appropriate for a cold planet, to radii $\sim 2-3 R_{\mathrm{J}}$ appropriate for young or inflated planets (e.g., Marley et al. 2007). Here $R_{\mathrm{J}}=7.192 \times 10^{9} \mathrm{~cm}$ is the equatorial radius of Jupiter.

\subsection{Evolutionary Calculations}

Figures 1 and 2 show evolutionary calculations for models with masses $M=0.2-20 M_{\mathrm{J}}$. All models were evolved for 20 Gyr. The initial models from create_initial_model had a large radius $R=5 R_{\mathrm{J}}$. The other parameters used are $Y=0.27$, $Z=0.02$ and $\alpha_{\text {MLT }}=2$. The opacity and EOS tables used are eos_file_prefix $=$ mesa, kappa_file_prefix $=$ gs 98 and kappa_lowT_prefix $=$ lowT_Freedman11. The atmosphere model is which_atm_option = simple_photosphere.

Figure 1 is a low mass extension of Figure 16 from Paper I, showing evolution in the $\rho_{\mathrm{c}}-T_{\mathrm{c}}$ plane. Each track (solid black curve) is labeled on the left with the planet's mass, and evolution goes from left to right. Initially the planet is non-degenerate and contraction increases both $\rho_{\mathrm{c}} \propto R^{-3}$ and $T_{\mathrm{c}} \propto R^{-1} \propto \rho_{\mathrm{c}}^{1 / 3}$. A maximum $T_{\mathrm{c}}$ is reached when $k_{\mathrm{B}} T_{\mathrm{c}} \sim E_{\mathrm{F}, \mathrm{c}}$, where $E_{\mathrm{F}, \mathrm{c}}$ is the electron Fermi energy at the center, beyond which $\rho_{\mathrm{c}}$ approaches a constant as $T_{\mathrm{c}}$ decreases further. Ignoring Coulomb interactions in the EOS, $S$ is a function of the electron degeneracy parameter $\mu_{\mathrm{e}} / k_{\mathrm{B}} T$, where $\mu_{\mathrm{e}}$ is the electron chemical potential and all models should have maximum $k_{\mathrm{B}} T_{\mathrm{c}} \sim E_{\mathrm{F}, \mathrm{c}}$ at the same $S$. The line labeled $S /\left(N_{\mathrm{A}} k_{\mathrm{B}}\right)=10.3$ indeed coincides with maximum $T_{\mathrm{c}}$ down to $M \simeq 1 M_{\mathrm{J}}$, but at smaller masses where non-ideal effects are more important, maximum $T_{\mathrm{c}}$ occurs when $S /\left(N_{\mathrm{A}} k_{\mathrm{B}}\right)<10.3$. Also shown in Figure 1 are lines of constant

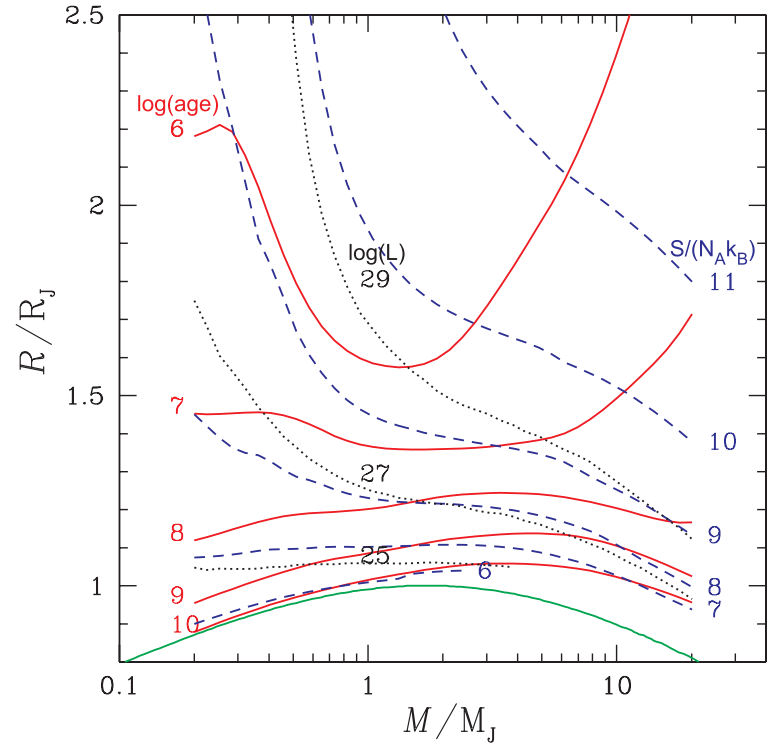

Figure 2. Radius vs. mass iso-contours from a suite of evolutionary calculations. The solid red lines show $R / R_{\mathrm{J}}$ vs. $M / M_{\mathrm{J}}$ at fixed values of $\log (\mathrm{age}[\mathrm{yr}])$, labeled on the left of each curve. The dashed blue curves are for fixed entropy, with each curve labeled by $S /\left(N_{\mathrm{A}} k_{\mathrm{B}}\right)$ on the right. The dotted black curves are for fixed luminosity, with each curve labeled by $\log \left(L\left[\mathrm{erg} \mathrm{s}^{-1}\right]\right)$ above $M=1 M_{\mathrm{J}}$. The green curve at the bottom is the $T=0 M-R$ relation from Zapolsky \& Salpeter (1969) for a solar mixture of $\mathrm{H}$ and $\mathrm{He}$.

(A color version of this figure is available in the online journal.)

age, shown as dotted red lines, and labeled on the bottom of the plot.

The same evolutionary calculations are used in Figure 2 to show radius versus mass at fixed values of age, entropy or luminosity. At late times, or low entropy and luminosity, the radius approaches the zero-temperature value (green curve; Zapolsky \& Salpeter 1969) for which thermal support is insignificant. The maximum radius occurs where gravitational and Coulomb energies, per ion, are comparable. The solid red lines, labeled by age on the left, show that contraction down to $R \simeq 1.5 R_{\mathrm{J}}$ is rapid, taking less than $10 \mathrm{Myr}$ for $M \lesssim 10 M_{\mathrm{J}}$. This initial rapid cooling phase occurs because the initial luminosity is orders of magnitude higher than the luminosity around one Gyr. This can been seen in the black dotted contours of constant $\log (L)$, where $L$ is larger by a factor of 100 for $R=1.3 R_{\mathrm{J}}$ and $10^{4}$ for $R=1.7 R_{\mathrm{J}}$, as compared to $R=1.1 R_{\mathrm{J}}$. The blue dashed lines show contours of constant entropy, labeled on the right by $S /\left(N_{\mathrm{A}} k_{\mathrm{B}}\right)$.

\subsection{Implementation of Inert Cores}

In the core accretion model of planet formation (e.g., Pollack et al. 1996; Hubickyj et al. 2005), a rock/ice core is first assembled. Once this core grows to $\sim 10 M_{\oplus}$, where $M_{\oplus}$ denotes an Earth mass, it can initiate rapid accretion of nebular gas, which could then dominate the mass of the planet. For studies of planetary radii, a central core composed of high mean molecular weight material can decrease the radius of the planet by a significant amount $\left(\simeq 0.1-0.2 R_{\mathrm{J}}\right)$. The MESAstar inert core feature allows one to add a core of specified mass $M_{\mathrm{c}}$ and radius $R_{\mathrm{c}}$, or more conveniently, density $\rho_{\mathrm{c}}$. A luminosity $L_{\mathrm{c}}$ may also be specified, although the high mean molecular weight of the core, as compared to the overlying $\mathrm{H} / \mathrm{He}$ envelope, implies that even large cores will tend to have small heat content (Fortney et al. 2006). This inert core is not presently evolved in any way, and changes in $P_{\mathrm{c}}$ during evolution are neglected as $R$ 
changes. While cores of mass $\lesssim 10-20 M_{\oplus}$ are commonly used for modeling solar system giants (e.g., Guillot 2005), the large masses and small radii of some exoplanets may imply far larger core masses (e.g., HD 149026; Sato et al. 2005). In addition, Neptune-like planets with smaller ratios of envelope to core masses may be modeled with MESA (Owen \& Wu 2013).

\subsection{Irradiation}

Surface heating by stellar irradiation changes the boundary condition for the planet's cooling and contraction. This modifies the planetary radius versus age for exoplanets at orbital separation $\lesssim 0.1 \mathrm{AU}$. MESA provides several ways to implement surface heating with varying degrees of fidelity to the true solution. These presently include:

1. An energy generation rate $\epsilon=F_{\star} / 4 \Sigma_{\star}$ applied in the outer mass column $\Sigma \leqslant \Sigma_{\star}$. Here $F_{\star}$ is the day-side flux from the star, and $\Sigma(r)=\int_{r}^{R} d r^{\prime} \rho\left(r^{\prime}\right)$ is the mass column. In steady-state, this generates an outward flux $F_{\star} / 4$, which is meant to simulate the angle-averaged flux over the planetary surface. This model implicitly assumes that day-night heat transport is efficient, and at the depths of interest the temperature is uniform over the surface. The parameters $F_{\star}$ and $\Sigma_{\star}$ are specified through the user-specified variables irradiation_flux and column_depth_for_irradiation, making this the simplest method to use. This heating mechanism represents absorption of stellar optical radiation well below the photosphere of the planet's thermal radiation and gives rise to greenhouse heating of the atmosphere where $\epsilon \neq 0$.

2. MESA's gray_irradiated atmosphere model (see also Appendix A.5) implements the angle-averaged temperature profile of Guillot (2010). This approximate solution to the transfer equation assumes two frequency bands: optical radiation from the star (with user-specified opacity $\kappa_{\mathrm{v}}$ ) and thermal radiation originating in the planet (with userspecified opacity $\kappa_{\text {th }}$ ). The temperature profile is derived using the Eddington approximation, assuming an external flux from the star as well as a flux from the planetary interior. While the Guillot (2010) model implemented in MESA uses a single temperature as a function of depth, it is derived allowing for local temperature variations over the surface which are then averaged over angle. This temperature profile is shown to be valid in the presence of horizontal heat transport by fluid motions. This is the only MESA atmosphere model that uses pressure instead of optical depth to determine the surface boundary condition. As this pressure may be relatively deep in the atmosphere, a correction to the radius may be required to give either the vertical thermal photosphere, or the optical photosphere in transit along a chord. Lastly, the relax_irradiation routine improves initial convergence by providing a starting model closer to the irradiated one.

3. Finally, MESA allows user-specified heating functions (e.g., $F_{\star}-\Sigma_{\star}$ surface heating) or atmosphere models (e.g., gray_irradiated). User-supplied routines may be easily implemented by using the other_energy module.

Figure 3 shows radius versus age for the planet HD 209458b (Guillot 2010). The two groupings of lines are for different heating depths, and within each grouping of lines, there are three calculations: MESA using the gray_irradiated surface boundary condition (solid red line), MESA using the $F_{\star}-\Sigma_{\star}$ surface heating profile (dashed blue line), and CEPAM

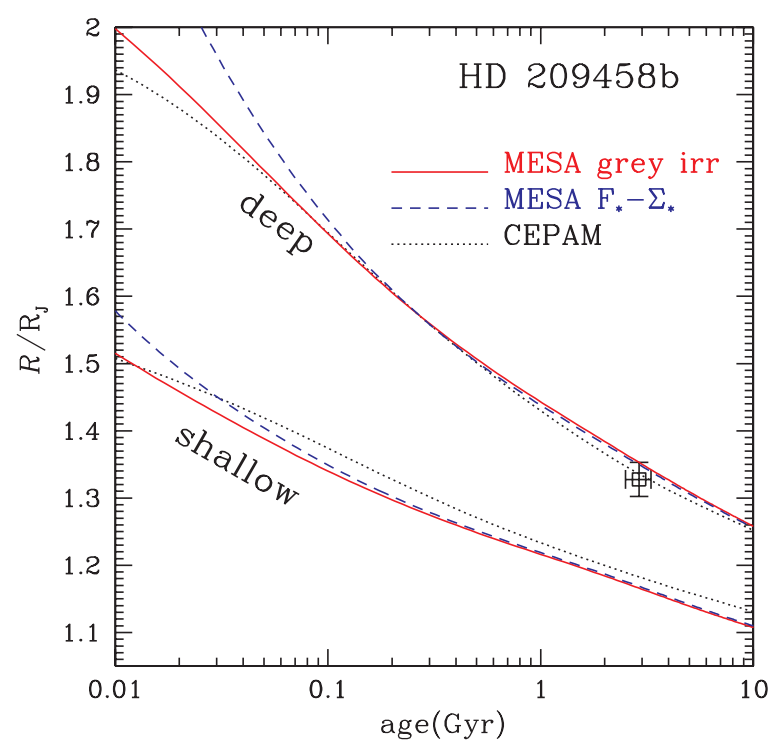

Figure 3. Radius vs. age for the planet HD 209458b. The solid red lines are for MESA, using the gray_irradiated atmosphere model. The dotted black lines show the CEPAM code results. The dashed blue lines show the MESA calculation using the $F_{\star}-\Sigma_{\star}$ surface heat source. The data point with error bars is the observed value of the radius for HD 209458b quoted in Guillot (2010). The two sets of curves are deep heating (upper three curves) and shallow heating (lower three curves).

(A color version of this figure is available in the online journal.)

(Guillot \& Morel 1995) using the same gray irradiated boundary condition (dotted black line; kindly provided by Tristan Guillot). The lower curves, corresponding to shallow heating, use fiducial values $\left(\kappa_{\text {th }}, \kappa_{\mathrm{v}}\right)=\left(10^{-2}, 6 \times 10^{-3}\right) \mathrm{cm}^{2} \mathrm{~g}^{-1}$ and give a model radius significantly smaller than the observed radius. The upper curves, corresponding to deep heating, use $\left(\kappa_{\mathrm{th}}, \kappa_{\mathrm{v}}\right)=\left(10^{-2}, 6 \times 10^{-4}\right) \mathrm{cm}^{2} \mathrm{~g}^{-1}$, yielding significantly hotter temperatures deep in the surface radiative zone, which slow the cooling enough to agree with the observed radius. The choice $\Sigma_{\star}=2 / \kappa_{\mathrm{v}}$ gives agreement between the gray irradiated and $F_{\star}-\Sigma_{\star}$ methods, where the factor of two accounts for the fact that the gray irradiated boundary condition has some heating below $\Sigma=1 / \kappa_{\mathrm{v}}$. The radii are at the $\tau_{\text {th }}=2 / 3$ photosphere for a vertical path into the atmosphere.

The agreement between all three methods is excellent at the $1 \%-2 \%$ level after 100 Myr. The remaining discrepancy between the MESA and CEPAM gray irradiated results are likely due to different opacity tables, with the MESA result using an update of Freedman et al. (2008) (R. S. Freedman 2011, private communication) while the CEPAM run uses the Allard et al. (2001) COND table. The differences at ages $\lesssim 100 \mathrm{Myr}$ are due to different starting conditions. The CEPAM calculation started with initial radius $2 R_{\mathrm{J}}$, whereas the MESA calculations started with $5 R_{\mathrm{J}}$. The MESA gray irradiated and $F_{\star}-\Sigma_{\star}$ calculations differ at $\lesssim 100$ Myr, likely because the former has a fixed thermal opacity while the latter allows the opacity to change.

\subsection{Low-mass Main Sequence Stars}

Most of MESAstar's capability to evolve low-mass $(M<$ $2 M_{\odot}$ ) stars was demonstrated in Section 7.1 of Paper I. MESA has seen use in the asteroseismology of helium core flashing stars (Bildsten et al. 2012) and the discovery of a new instability from the onset of ${ }^{3} \mathrm{He}$ burning (van Saders \& Pinsonneault 2012). We expect the future use of MESAstar for asteroseismic investigations of these stars to be substantial (see Section 3). 


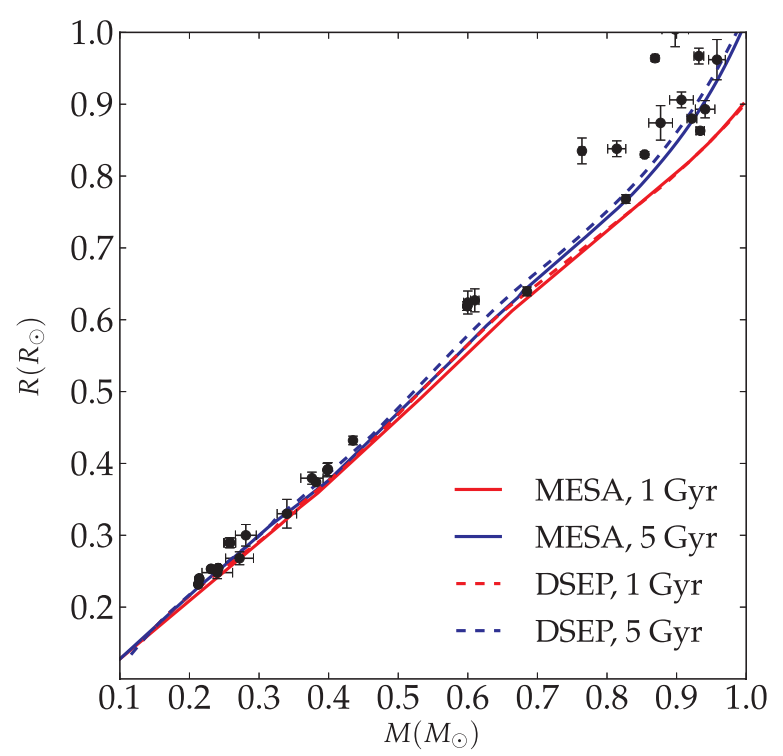

Figure 4. Stellar isochrones at solar composition spanning $0.1-1 M_{\odot}$ from MESAstar (solid lines) and Dotter et al. (2008, dashed lines) in the mass-radius plane. The data points plotted are the same as shown by Bass et al. (2012).

(A color version of this figure is available in the online journal.)

The derivation of accurate planetary radii based on transits requires accurate radii of the host stars; this motivates MESAstar investigations of low-mass stars (Lloyd 2011). Figure 4 shows 1 and 5 Gyr isochrones at solar composition $(Y=0.27, Z=$ 0.019) from MESAstar (solid lines) and Dotter et al. (2008, dashed lines) in the mass-radius diagram. Data points shown in Figure 4 are taken from Torres et al. (2010), Carter et al. (2011), Irwin et al. (2011), and Bass et al. (2012). This figure is a reproduction of the upper panel of Figure 11 from Bass et al. (2012). Figure 4 indicates that MESAstar is capable of producing mass-radius relations for MS stars that are consistent with other widely used models as well as observational data. The MESAstar models were computed using, as much as possible, the same physical assumptions as the models used by Dotter et al. (2008). The main difference is the EOS, for which Dotter et al. (2008) used FreeEOS ${ }^{10}$ and MESAstar uses a combination

\footnotetext{
10 http://freeeos.sourceforge.net
}

of the OPAL (Rogers \& Nayfonov 2002) and SCVH EOS for thermodynamic parameters relevant to this diagram.

\section{ASTEROSEISMOLOGY}

With its highly configurable output options and its ability to calculate asteroseismic variables, MESAstar can readily produce models suitable for use with a range of oscillation codes. In addition to its own text output files, MESA can produce outputs in formats widely used by stellar oscillation codes, such as fgong and osc (Monteiro 2009).

In Figure 5 we show the evolution of a $1 M_{\odot}$ model in the Hertzsprung-Russell (H-R) diagram and in $T_{\mathrm{c}}-\rho_{\mathrm{c}}$ space. These were evolved following the test case found in 1M_pre_ms_to_wd, which was modified to include diffusion. This runs without user intervention from PMS to WD. To demonstrate the changing stellar structure as the model evolves from the MS to post helium-core burning on the asymptotic giant branch (AGB), we show in Figure 6 some of the fundamental quantities extracted from the corresponding profile.data files for the models marked in Figure 5. These include the Lamb and Brunt-Väisälä frequencies defined respectively by

$$
\begin{aligned}
S_{\ell}^{2} & =\frac{\ell(\ell+1) c_{\mathrm{s}}^{2}}{r^{2}}, \\
N^{2} & =\frac{g}{r}\left[\frac{1}{\Gamma_{1}} \frac{d \ln P}{d \ln r}-\frac{d \ln \rho}{d \ln r}\right],
\end{aligned}
$$

where $c_{\mathrm{s}}$ is the adiabatic sound speed and $\ell$ is the spherical harmonic degree.

\subsection{The Solar Sound Speed Profile}

The seismic properties of the Sun provide a test of stellar evolution models, and an opportunity to calibrate $\alpha_{\mathrm{MLT}}$ for any particular set of input physics and other assumptions. The MESAstar test case solar_calibration produces a calibrated Standard Solar Model. Figure 7 shows the difference between the helioseismically inferred solar sound speed profile and this model. We also show "Model S" from Christensen-Dalsgaard et al. (1996). Both models employ comparable input physics
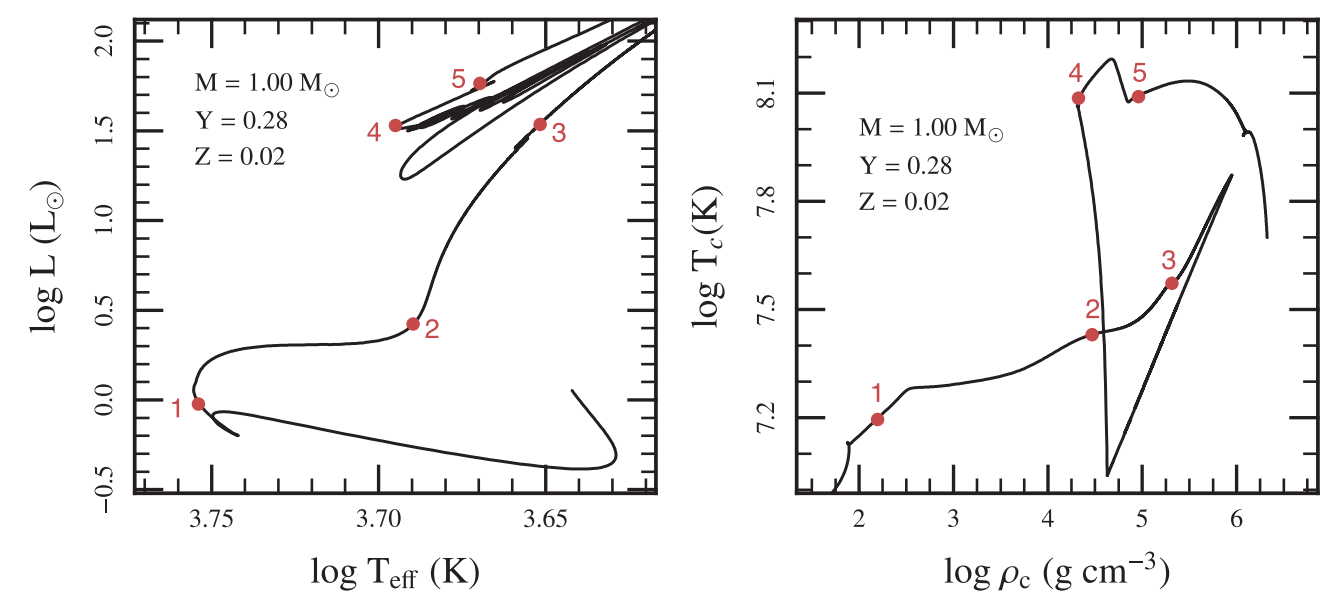

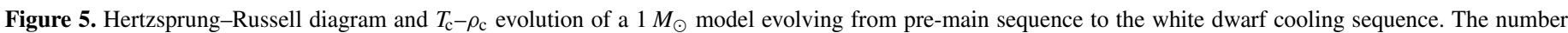
labels denote selected models, for which we show internal profiles in Figure 6.

(A color version of this figure is available in the online journal.) 

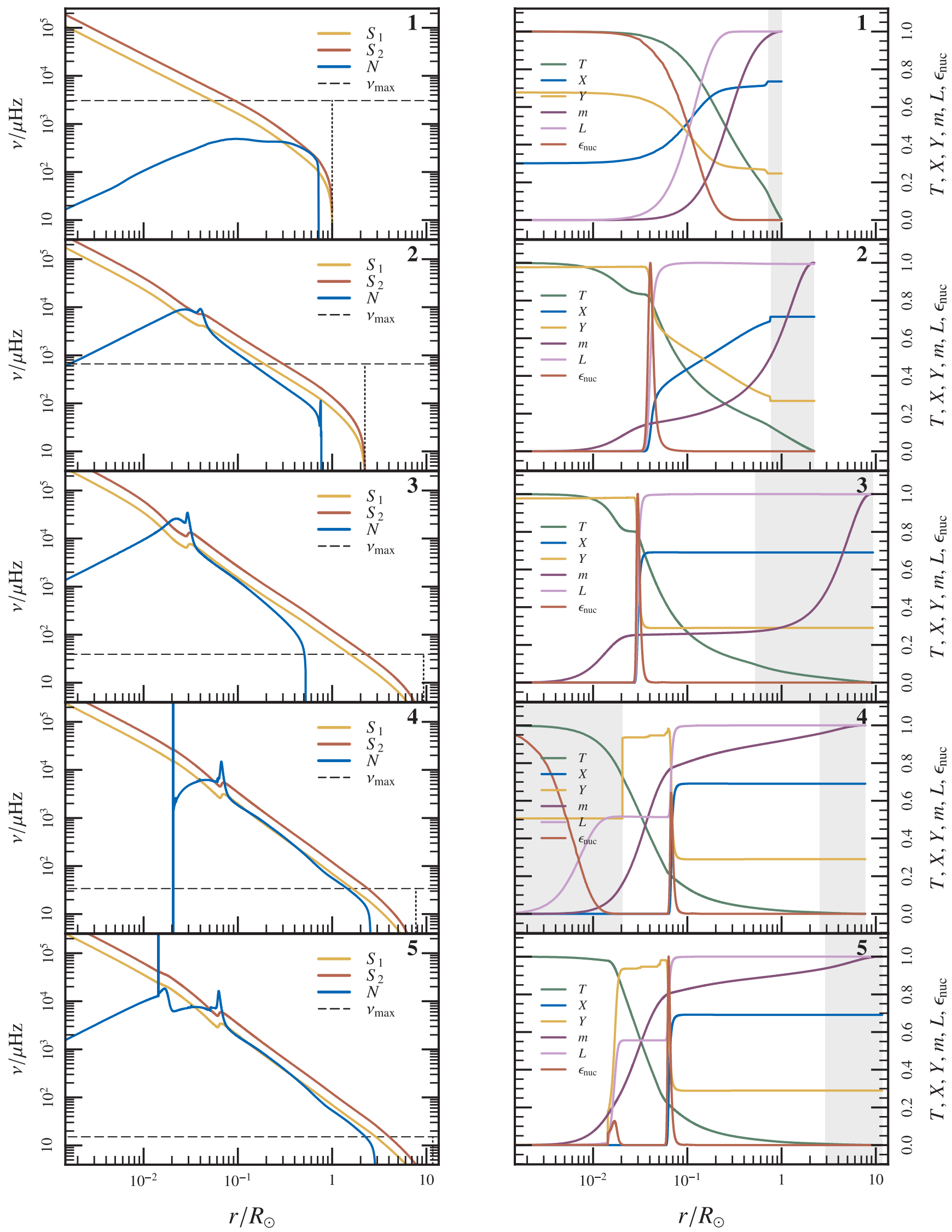

Figure 6. Internal structure of the five points (indicated by the numbers in each panel) marked in Figure 5. The left panel for each point shows $N$ and $S_{\ell}$ for harmonic degrees $\ell=1$ and 2 . The dashed line indicates the frequency of maximum power $v_{\max }$ of the stochastically excited solar-like modes. The vertical dotted lines mark the radius of the model. Right panels show temperature, hydrogen and helium mass fractions, mass, luminosity, and the nuclear energy generation rate. Gray areas mark convective regions according to the Schwarzschild criterion.

(A color version of this figure is available in the online journal.) 


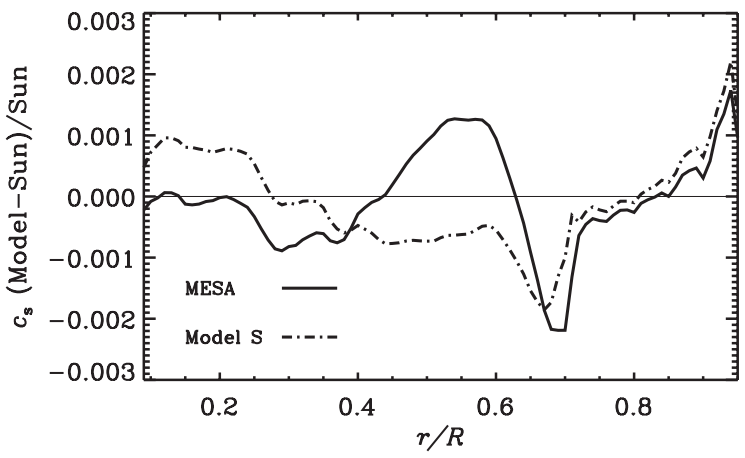

Figure 7. Comparison of the difference between the helioseismically inferred sound speed profile (Bahcall et al. 1998) of a MESAstar model and Model S (Christensen-Dalsgaard et al. 1996).

and assume solar abundances from Grevesse \& Noels (1993) and Grevesse \& Sauval (1998). One clear improvement since Paper I is a smoother sound speed profile at small $r / R$, which is primarily due to improvements in the diffusion module. This is particularly important for asteroseismology, where sharp features in the sound speed profile can influence the stellar oscillation frequencies. The results are based on the solar calibration test case compiled with the GNU Fortran compiler version 4.7.2 on Mac OS X 10.7.5; Appendix B.11 provides information about how the solar calibration results may depend on different operating systems and compilers.

\subsection{New Asteroseismic Capabilities in MESA}

The "astero" extension to MESAstar implements an integrated approach that passes results automatically between MESAstar and the new MESA module based on the adiabatic code ADIPLS (Christensen-Dalsgaard 2008a, 2011 June release). The MESA module ADIPLS also supports independent use for post-processing, including the calculation of pulsation frequencies.

This astero extension enables calculation of selected pulsation frequencies by MESAstar during the evolution of the model. This allows fitting to the observations that can include spectroscopic constraints (e.g., $[\mathrm{Fe} / \mathrm{H}]$ and $T_{\text {eff }}$ ), asteroseismic constraints, such as the large frequency separation $(\Delta \nu)$ and the frequency of maximum power $\left(\nu_{\max }\right)$, and even individual frequencies. A variety of approaches for finding a best-fitting model are available, including grid searches and automatic $\chi^{2}$ minimization by the Hooke-Jeeves algorithm (Hooke \& Jeeves 1961) or by the "Bound Optimization BY Quadratic Approximation" (Powell 2009) technique. These searches are user controlled through a number of parameter bounds and step sizes. Users also have full control over the relative weight assigned to the seismic and spectroscopic parts of the $\chi^{2}$ statistic.

For the automated $\chi^{2}$ minimization, astero will evolve a PMS model from a user defined starting point, and find the best match along that single evolutionary track. The code then recalculates the track, again initiated at the PMS, with different initial parameters such as mass, composition, mixing length parameter and overshoot, and repeats until the lowest $\chi^{2}$ has been found.

Calculating specific mode frequencies is computationally intensive. Hence, a number of options exist to improve the efficiency of the minimization when individual frequencies are included. Bounds can be established on stellar parameters (e.g., $T_{\text {eff }}$, central $\mathrm{H}$ mass fraction, $\left.\Delta v\right)$, so that ADIPLS is invoked only when the model falls within these bounds. This enables certain evolutionary stages to be skipped when other observational diagnostics rule them out-if a star is known to be a red giant, for instance, there is no sense in invoking ADIPLS when models are on the MS. The large frequency separation, $\Delta v$, of the model is calculated as the inverse of the sound travel time through the star, $\Delta v=\left[2 \int d r / c_{\mathrm{s}}\right]^{-1}$ (Tassoul 1980; Gough 1986). There is also the option to derive $\Delta v$ using simple solar scaling: $\Delta v \propto\left(M / R^{3}\right)^{0.5}$ (Kjeldsen \& Bedding 1995). To obtain $v_{\max }$, MESA scales the solar value with the acoustic cut-off frequency: $v_{\max } \propto g / \sqrt{T_{\text {eff }}}$ (Brown et al. 1991; Kjeldsen \& Bedding 1995).

Moreover, hierarchical approaches to the frequency fitting can be selected, saving large amounts of computational time. In one case the radial modes are first calculated, and only when they match reasonably well are the non-radial mode frequencies derived and included in the $\chi^{2}$. This is particularly beneficial for red giants where the calculation of the non-radial frequencies is extremely time consuming. Another example is when the time steps in the stellar evolution calculations are too large to find an accurate minimum of $\chi^{2}$. Hence, as a further option to increase efficiency while attaining accuracy, the time steps can be set to automatically reduce when the model comes close to the "target box" of the observational constraints. As for other modules used in MESAstar, astero offers a range of graphical outputs including an échelle diagram where the fitting process can be followed in real time.

There is also an option for including corrections to the model frequencies on the fly to compensate for the inadequate modeling of the near surface layers of the star. The effect, known as the "surface term," is seen as a frequency dependent offset between the modeled and observed acoustic frequencies of the Sun (e.g., Christensen-Dalsgaard \& Thompson 1997). The offset increases toward higher frequencies and is well described by a power law (Kjeldsen et al. 2008). MESAstar follows the approach described by Kjeldsen et al. (2008) for correcting the surface term.

To illustrate the performance of astero, we show here a fit to the star HD 49385. The input frequencies and the spectroscopic constraints are from Deheuvels et al. (2010). We first ran a wide-range grid search over $M, \alpha_{\mathrm{MLT}},[\mathrm{Fe} / \mathrm{H}]$, and $Y$, including only $[\mathrm{Fe} / \mathrm{H}], T_{\text {eff }}$, and $\Delta v$ as observational constraints. The results of this initial search guided our starting parameters and ranges for the next automatic $\chi^{2}$ minimization. We first compare our grid results with those of the RADIUS grid search routine (Stello et al. 2009), which is based on a grid of ASTEC models (Christensen-Dalsgaard 2008b) and find agreement within uncertainties.

We then include the individual oscillation frequencies and use the Hooke-Jeeves algorithm for the $\chi^{2}$ minimization. Model frequencies were corrected for the surface term, and the part of the $\chi^{2}$ coming from the frequencies was given two-thirds of the weight in the final $\chi^{2}$, similar to that used by Metcalfe et al. (2012). To ensure we adequately sample the parameter space, we initiate the search at several initial values within a broad range. By starting the search from multiple initial values, we aim to reduce the chance of ending up in a local minimum, which could potentially provide unphysical results, such as the spuriously low helium abundances reported by Mathur et al. (2012). Current developments in astero further seeks to overcome such problems and improve the robustness of the results by including frequency ratios (Roxburgh \& Vorontsov 2003; Silva Aguirre et al. 2013) in the $\chi^{2}$ minimization.

Each "Hooke" search generates several stellar evolution tracks, each with a best $\chi^{2}$ value. We then combine the data from 


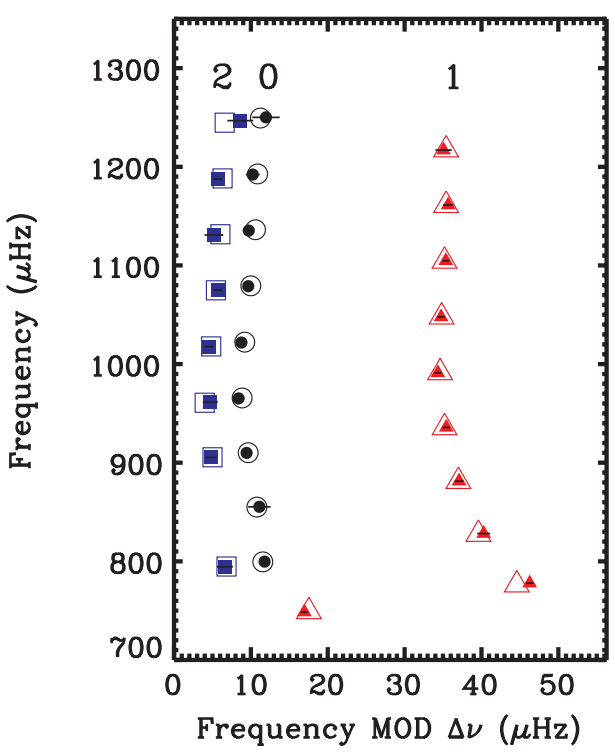

Figure 8. Échelle diagram of the oscillation frequencies of the subgiant HD 49385. Observed frequencies are shown with filled symbols as blue squares $(\ell=2)$, black circles $(\ell=0)$, and red triangles $(\ell=1)$, and the matched model frequencies are shown with open symbols. Black horizontal lines indicates $1 \sigma$ error bars.

(A color version of this figure is available in the online journal.)

Table 2

Properties of Best Fitting Model to HD 49385

\begin{tabular}{lc}
\hline \hline Quantity & Value \\
\hline$M / M_{\odot}$ & $1.30 \pm 0.04$ \\
$R / R_{\odot}$ & $1.972 \pm 0.016$ \\
$L / L_{\odot}$ & $4.9 \pm 0.4$ \\
$\log g$ & $3.962 \pm 0.003$ \\
$T_{\text {eff }} / \mathrm{K}$ & $6115 \pm 125$ \\
$\mathrm{Age} / \mathrm{Gyr}$ & $4.1 \pm 0.4$ \\
$\alpha_{\mathrm{MLT}}$ & $1.9 \pm 0.1$ \\
{$[\mathrm{Fe} / \mathrm{H}]_{i}$} & $0.15 \pm 0.04$ \\
{$[\mathrm{Fe} / \mathrm{H}]_{s}{ }^{\mathrm{a}}$} & 0.063 \\
$Y_{\text {initial }}$ & $0.29 \pm 0.02$ \\
$Z_{\text {initial }}$ & 0.0222 \\
$\chi^{2}$ & 2.40 \\
\hline
\end{tabular}

Note. ${ }^{\mathrm{a}}[\mathrm{Fe} / \mathrm{H}]_{s}$ is the log of the ratio of the surface $(Z / X)$ relative to the solar value of 0.02293 .

about 1400 tracks to estimate the $1 \sigma$ uncertainties in the varied parameters following the approach by Deheuvels et al. (2010). The lowest (reduced) $\chi^{2}$ value we obtained was 2.4 with a few tens of models in the 2.4-4.0 range, which all fit the frequencies similarly well. Among these models there are two families of results, one of which has slightly lower $[\mathrm{Fe} / \mathrm{H}]$ and $Y$, and a slightly increased value for the spectroscopic part of the $\chi^{2}$.

The comparison of the observed and modeled frequencies for the realization with the lowest $\chi^{2}$ is shown in the échelle diagram format in Figure 8. A plot of the internal structure including the Brunt-Väisälä and Lamb frequencies is shown in Figure 9, and the parameters of the model are listed in Table 2. We set $f_{\text {ov }}=0.015$ and use the GN98 solar abundances. Our results can be best compared to those listed as "low $\alpha_{\text {ov }}$ " and "GN93" in Table 4 of Deheuvels \& Michel (2011) and agree within the uncertainties.

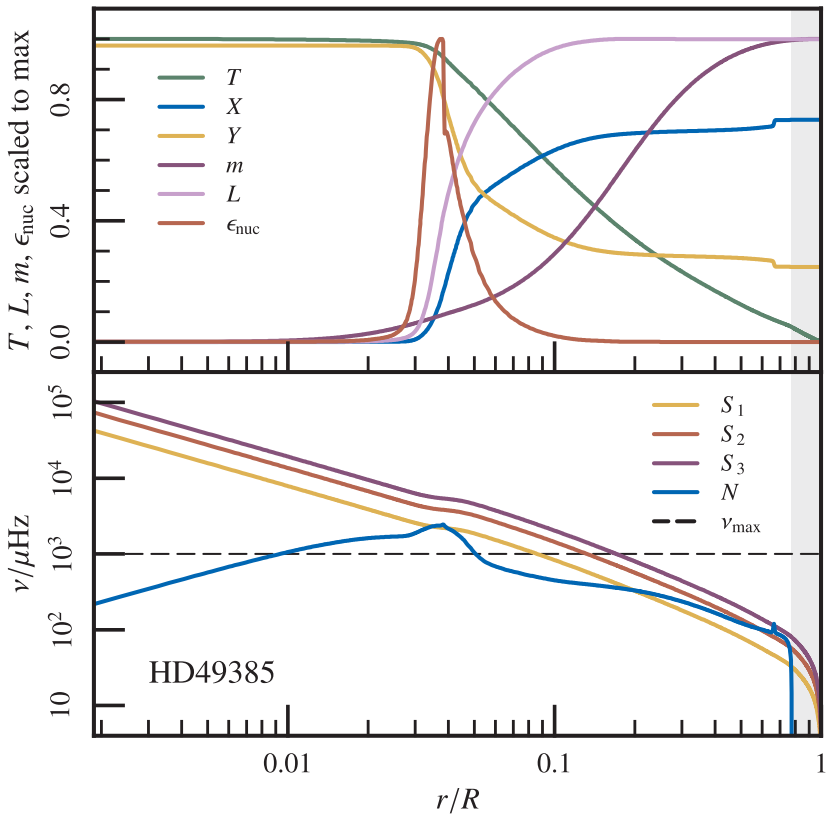

Figure 9. Same format as in Figure 6, but for the best-fitting model of HD 49385 (see also Table 2).

(A color version of this figure is available in the online journal.)

\subsection{The Effect of Composition Gradients on the Brunt-Väisälä Frequency}

Including the effect of composition gradients in the calculation of the Brunt-Väisälä frequency is important for two reasons. First, it is necessary for implementing the Ledoux criterion for convection, which is used to determine the chemical mixing and convective heat transport in a region (see Section 4.1). Second, a smooth and accurate method for calculating $N^{2}$ is crucial for studies of $g$-mode pulsation in stars. In a highly degenerate environment, the pressure is nearly independent of temperature, and $P \propto \rho^{\Gamma_{1}}$, so from Equation (4) we see that $N^{2}$ depends on the difference of two large and nearly equal quantities. This can lead to a loss of precision and a noisy $N^{2}$. To eliminate this problem, $N^{2}$ is rewritten into a form that depends on the difference of the adiabatic and true temperature gradients and on the composition gradient:

$$
N^{2}=\frac{g^{2} \rho}{P} \frac{\chi_{T}}{\chi_{\rho}}\left(\nabla_{\mathrm{ad}}-\nabla_{T}+B\right) .
$$

The term $B$ explicitly takes into account the effect of composition gradients and is commonly called the Ledoux term (e.g., Unno et al. 1989; Brassard et al. 1991). For the general case of an $N$-component plasma with mass fractions $\left\{X_{i}\right\}$, the standard formula for $B$ is (e.g., Unno et al. 1989)

$$
B=-\frac{1}{\chi_{T}} \sum_{i=1}^{N-1}\left(\frac{\partial \ln P}{\partial \ln X_{i}}\right)_{\rho, T,\left\{X_{j \neq i}\right\}} \frac{d \ln X_{i}}{d \ln P} .
$$

Since $\sum_{i=1}^{N} X_{i}=1$, one of the mass fractions can be eliminated, so that the sum in Equation (6) runs from 1 to $N-1$. We note that the partial derivatives in Equation (6) hold all the $\left\{X_{j}\right\}$ constant except for $X_{i}$ and $X_{N}$, where $X_{N}$ is varied so as to maintain $\sum_{i=1}^{N} X_{i}=1$.

Although Equation (6) is correct as written, we have developed a new, formally equivalent prescription that is both numerically robust and simpler to implement. We define a new 


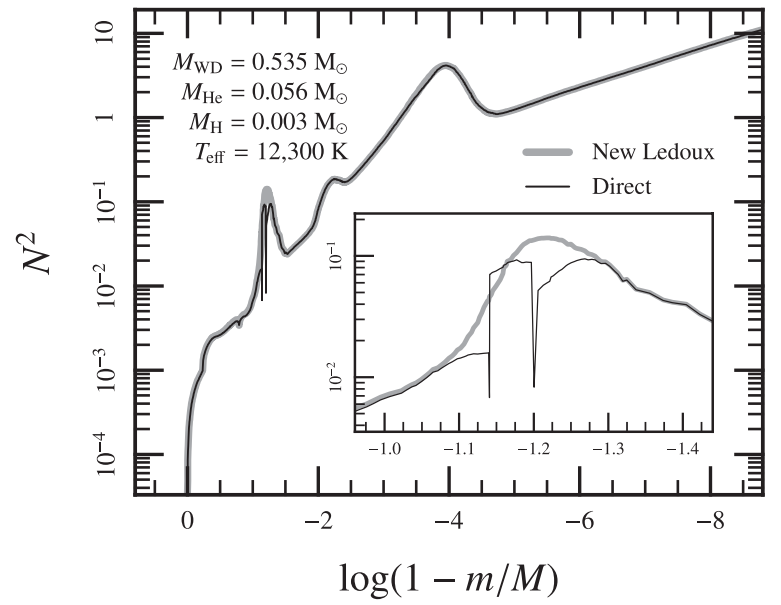

Figure 10. Comparison of the new Ledoux prescription for $N^{2}$ (Equations (5) and (8)) vs. the direct numerical calculation (Equation (4)). This calculation is for a $0.535 M_{\odot}$ white dwarf model at $T_{\text {eff }}=12,300 \mathrm{~K}$.

Ledoux term by taking a directional derivative along the radial composition gradient in the stellar model,

$$
\begin{aligned}
B \equiv & -\frac{1}{\chi_{T}} \lim _{\delta \ln P \rightarrow 0} \\
& \times \frac{\ln P(\rho, T, \boldsymbol{X}+(d \boldsymbol{X} / d \ln P) \delta \ln P)-\ln P(\rho, T, \boldsymbol{X})}{\delta \ln P} .
\end{aligned}
$$

The implementation of the above derivative typically involves the use of quantities on neighboring mesh points. Using the subscript $k$ to denote the value of a given quantity on the $k$ th mesh point, we therefore have

$$
B=-\frac{1}{\chi_{T}} \frac{\ln P\left(\rho_{k}, T_{k}, X_{k+1}\right)-\ln P\left(\rho_{k}, T_{k}, X_{k}\right)}{\ln P_{k+1}-\ln P_{k}} .
$$

This is the form of the Ledoux term that is implemented in MESA and we term it the "new Ledoux" formulation. Since MESA ensures that $\sum_{i=1}^{N} X_{i}=1$ at each mesh point, this condition does not have to be separately enforced. This formulation requires just one numerical difference along $\boldsymbol{X}$ that is consistent with the stellar model and EOS. Because MESA's EOS does not directly supply the partial derivatives required for the formulation in Equation (6), an implementation of that method would suffer in both accuracy and efficiency from having to do a large number of numerical differences. Brassard et al. (1991) dealt with a similar problem by using a restricted form of Equation (6) that included only the helium composition gradient. They showed that for cases where their restricted form applied, it gave significantly better numerical results than an implementation of Equation (4) based on finite differences. Figure 10 shows that our new Ledoux prescription (gray heavy curve) retains their good results compared to Equation (4) (thin black curve) while extending the applicability to cases that cannot be dealt with using only helium gradients.

\section{MIXING MECHANISMS INVOLVING COMPOSITION GRADIENTS}

We described the implementation of mixing-length theory (MLT) in Paper I, including the allowance for overshoot beyond the boundaries of the convective zones as determined by the standard Schwarzschild condition, $\nabla_{\mathrm{rad}}>\nabla_{\mathrm{ad}}$. Overshooting is implemented via an exponential decay of the convective diffusion coefficient beyond the boundary of full convection, following Herwig (2000):

$$
D_{\mathrm{ov}}=D_{\text {conv }, 0} \exp \left(-\frac{2 \Delta r}{f_{\mathrm{ov}} \lambda_{P}}\right),
$$

where $D_{\text {conv, } 0}$ is the diffusion coefficient at the convective border, $\Delta r$ is the distance from the start of overshoot, and $\lambda_{P}$ is the local pressure scale height. The user-adjusted dimensionless parameter $f_{\text {ov }}$ then determines the extent of the overshooting region. MESA also allows for the adoption of a step-function overshooting model, where the mixing region extends a distance $f_{\text {ov }} \lambda_{P}$ beyond the convective boundary with a constant specified diffusion coefficient.

In Paper I we did not implement the influence of composition gradients on mixing and the resulting diffusion coefficients when instabilities are operative. The description of how MESAstar calculates the Ledoux criterion is in Section 3.3. In this section, we describe the implementation of mixing due to composition gradients in stellar interiors.

We refer to Appendix B.7 for a discussion of the free parameters involved in the implementation of these mixing mechanisms.

\subsection{Semiconvection}

Semiconvection refers to mixing in regions unstable to Schwarzschild but stable to Ledoux, that is

$$
\nabla_{\mathrm{ad}}<\nabla_{T}<\nabla_{\mathrm{L}}
$$

where $\nabla_{\mathrm{L}}$ is the sum of the adiabatic gradient and the Brunt composition gradient term (see Equations (5) and (8)),

$$
\nabla_{\mathrm{L}} \equiv \nabla_{\mathrm{ad}}+B
$$

Once $\nabla_{\mathrm{L}}$ is calculated, regions satisfying Equation (10) undergo mixing via a time-dependent diffusive process with a diffusion coefficient calculated by the mlt module following Langer et al. (1983),

$$
D_{\mathrm{sc}}=\alpha_{\mathrm{sc}}\left(\frac{K}{6 C_{P} \rho}\right) \frac{\nabla_{T}-\nabla_{\mathrm{ad}}}{\nabla_{\mathrm{L}}-\nabla_{T}},
$$

where $K=4 a c T^{3} /(3 \kappa \rho)$ is the radiative conductivity, $C_{P}$ is the specific heat at constant pressure, and $\alpha_{\mathrm{sc}}$ a dimensionless efficiency parameter. See Appendix B.7 for a discussion of the range of values for $\alpha_{\text {sc }}$.

We stress that semiconvection and overshooting have distinct implementations in MESA. Both are time-dependent diffusive processes. As an example, in Figure 11 we display profiles of thermodynamic gradients and their resulting diffusion coefficients during core helium burning in a semiconvective model with $\alpha_{\mathrm{sc}}=0.01$ and in an exponentially overshooting model with $f_{\mathrm{ov}}=10^{-5}$.

\subsection{Thermohaline Mixing}

Thermohaline mixing arises in the presence of an inversion of the mean molecular weight in regions that are formally stable against convection according to the Ledoux criterion,

$$
\nabla_{T}-\nabla_{\mathrm{ad}} \leqslant B \leqslant 0
$$




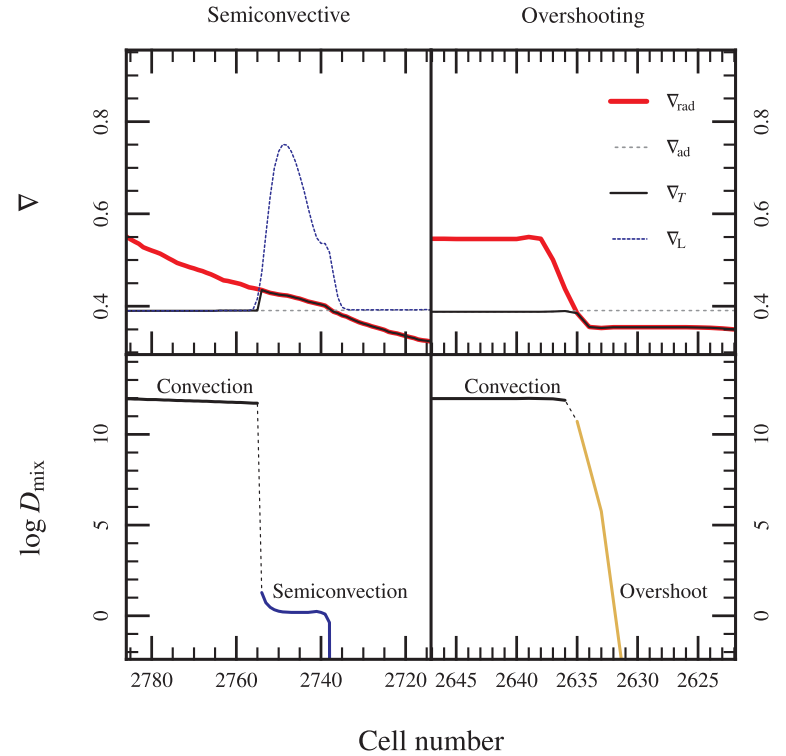

Figure 11. Sample profiles of semiconvective (left) and exponentially overshooting (right) $3 M_{\odot}$ models undergoing core helium burning. Top panels show the radiative, adiabatic, temperature, and Ledoux gradients that determine mixing boundaries and diffusion coefficients. Bottom panels show the resulting diffusion coefficients for energy and chemical transport. In either case, a thin dotted line spanning a single intermediate cell joins the convective and semiconvective/overshoot curves. This is intended merely as a guide for the eye, as diffusion coefficients are defined only at the two boundaries of a cell. In particular, diffusion for this intermediate cell is governed by convection at its interior boundary and semiconvection/overshoot at the exterior. The semiconvective model shown here was run with $\alpha_{\mathrm{sc}}=0.01$, the exponentially overshooting model with $f_{\mathrm{ov}}=10^{-5}$. The profiles are taken at the points marked in Figure 15.

(A color version of this figure is available in the online journal.)

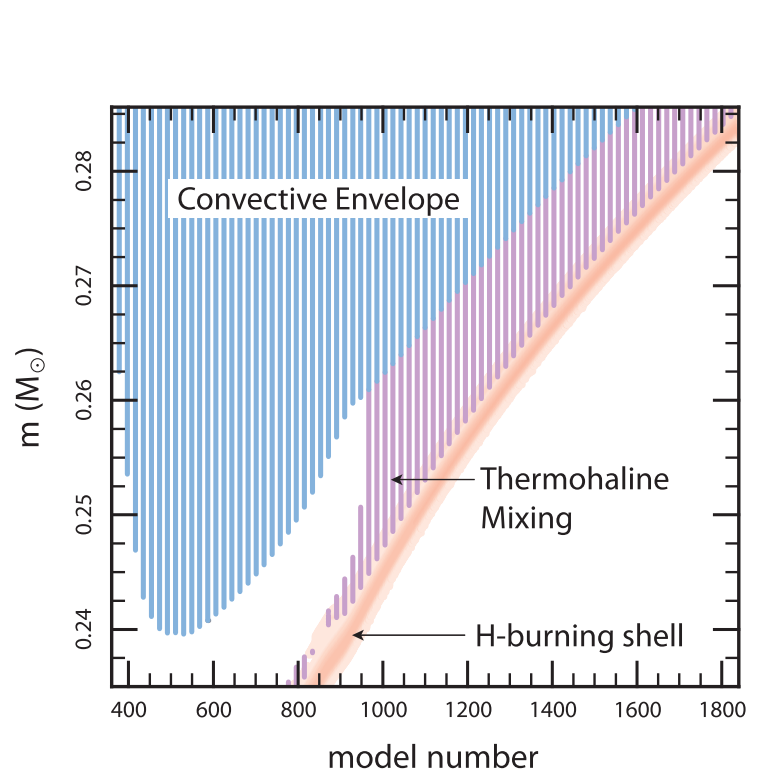

In MESA thermohaline mixing is treated in a diffusion approximation, with a diffusion coefficient motivated by the linear stability analysis of Ulrich (1972) and Kippenhahn et al. (1980)

$$
D_{\text {th }}=\alpha_{\text {th }} \frac{3 K}{2 \rho C_{P}} \frac{B}{\left(\nabla_{T}-\nabla_{\mathrm{ad}}\right)} .
$$

The quantity $\alpha_{\mathrm{th}}$ is a dimensionless efficiency parameter. In the linear analysis it depends on the aspect ratio of the blobs/fingers arising from the instability. In the case of salt fingers such a value is calibrated using laboratory experiments in water (e.g., Krishnamurti 2003), where the fingers have an aspect ratio of $\approx 5$. In the stellar case the value of this parameter is vexatious (e.g., Charbonnel \& Zahn 2007; Denissenkov \& Pinsonneault 2008; Cantiello \& Langer 2010; Wachlin et al. 2011), with recent 2D and 3D hydrodynamical calculations pointing toward a much reduced value of $\alpha_{\text {th }}$ relative to the salt fingers case (Denissenkov 2010; Traxler et al. 2011; Brown et al. 2013). Figure 12 shows a calculation including the effects of thermohaline mixing during the RGB phase of a $1 M_{\odot}$ star after the luminosity bump (e.g., Charbonnel \& Zahn 2007; Cantiello \& Langer 2010). For this calculation a value $\alpha_{\text {th }}=2$ has been adopted, but see Appendix B.7 for a discussion of the range of options.

\subsection{Impact of Mixing on Convective Core Hydrogen and Helium Burning}

The duration of the hydrogen and helium core burning depends on the extent of the convective core, so we focus here on exhibiting the MESA capabilities during these phases. As we noted above, there are many physical effects that change the size of the convective core, such as semiconvection, overshooting, and rotation-induced mixing. For example, the Schwarzschild criterion implies larger cores than the Ledoux criterion, but when using Ledoux alone, the region above the convective boundary is overstable and so semiconvection occurs (see Section 4.1).

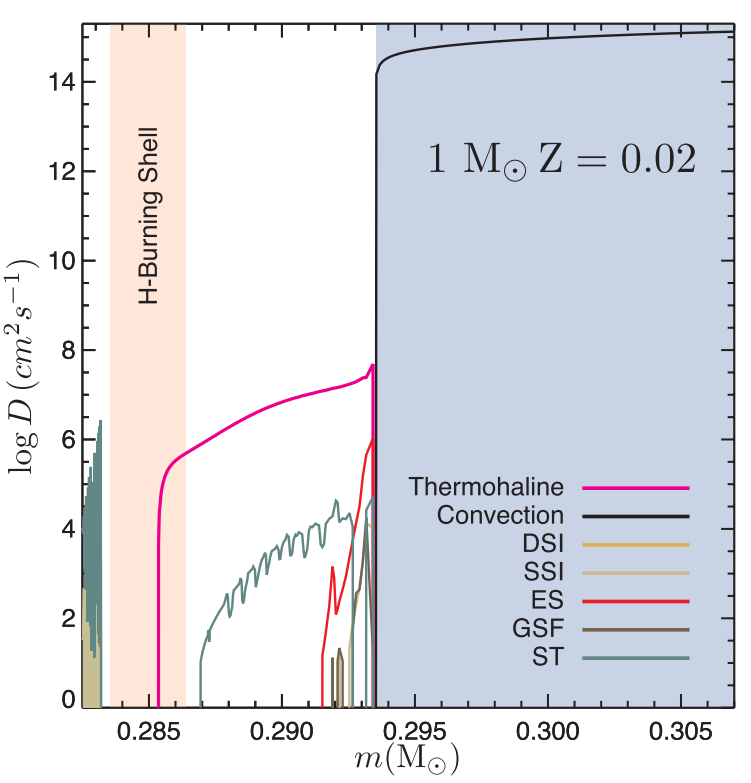

Figure 12. Thermohaline mixing during the RGB phase of a $Z=0.02,1 M_{\odot}$ model, initially rotating with an equatorial velocity of $10 \mathrm{~km} \mathrm{~s}^{-1}$ and adopting $\alpha_{\mathrm{th}}=2$. In the left panel a Kippenhahn diagram shows, in mass coordinate and as function of model number, the locations of the retreating convective envelope (blue), of the H-burning shell (red), and of the thermohaline mixing region (magenta). The right panel shows diffusion coefficient profiles extracted at model number 1849, which is the last model shown in the Kippenhahn plot. The H-burning shell and the convective envelope are shaded in red and blue, respectively. Thermohaline mixing (magenta line) transports chemicals between the burning shell and the convective envelope. Also shown are the diffusion coefficients resulting from Eddington-Sweet (ES) circulation, magnetic torques by dynamo-generated fields (ST), dynamical shear (DSI), secular shear (SSI), and Goldreich-Schubert-Fricke (GSF) instability (see Section 6 for details).

(A color version of this figure is available in the online journal.) 


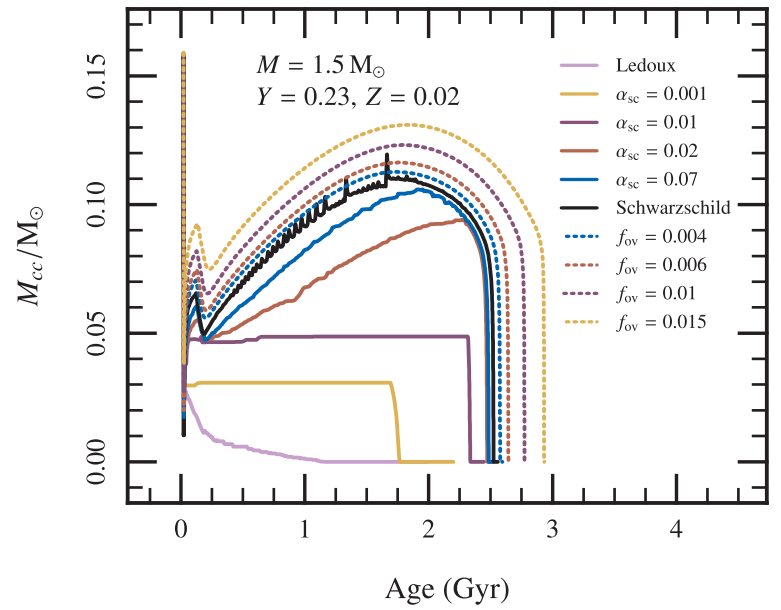

Figure 13. History of convective core extent during the main sequence for a nonrotating $1.5 M_{\odot}$ star with various mixing options. The plot shows the boundary of convection not including the extent of semiconvection or overshooting. (A color version of this figure is available in the online journal.)

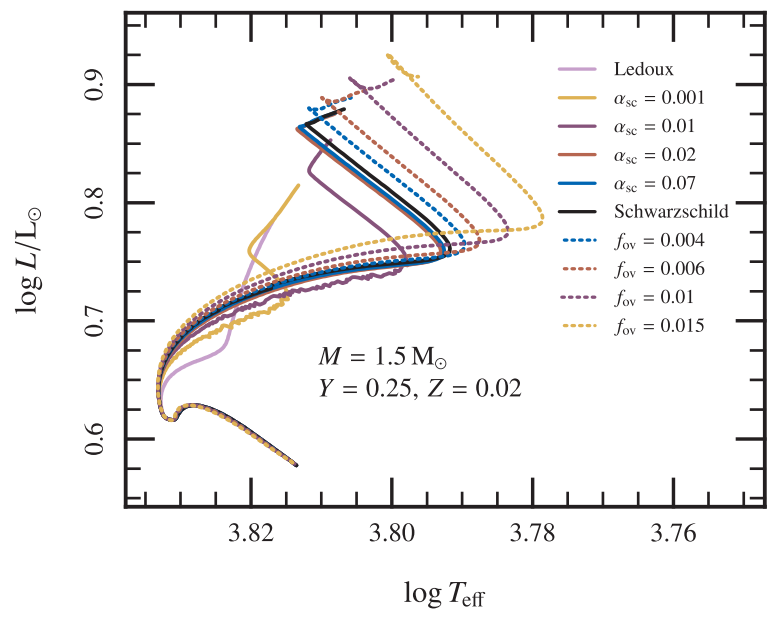

Figure 14. H-R diagram for the non-rotating $1.5 M_{\odot}$ star with various mixing options. Tracks are displayed from ZAMS until depletion of core hydrogen to $X=10^{-5}$.

(A color version of this figure is available in the online journal.)

We evolved a non-rotating $1.5 M_{\odot}$ star with $(Y, Z)=(0.23$, 0.02 ) through central hydrogen burning using Ledoux, Ledoux plus semiconvection, Schwarzschild, and Schwarzschild plus overshoot. As is evident in Figure 13, this set of physical processes leads to a large range of convective core masses and thereby MS lifetimes. For the parameters explored we found that overshooting increases the lifetime by a factor $\lesssim 1.2$ for Schwarzschild and $\lesssim 2.5$ for Ledoux. Figure 14 shows an $\mathrm{H}-\mathrm{R}$ diagram for each of the $1.5 M_{\odot}$ models undergoing core hydrogen burning, showing the impact of convective core extent on MS turnoff morphology.

We also evolved a non-rotating $3 M_{\odot}$ star with $(Y, Z)=$ $(0.25,0.02)$ through central helium burning. Overshooting extends the burning lifetime by a factor $\lesssim 1.6$ for Schwarzschild and $\lesssim 2.8$ for Ledoux (see Figure 15). Although this lengthening of the core burning phase is always true of convective overshoot, we find that the extension of the overshoot and convective regions is sensitive to the temporal resolution adopted. With sufficiently large values of $f_{\text {ov }}$, the upper boundary develops oscillatory behavior which can also affect the lifetime. This behavior also occurs with the step-function implementa-

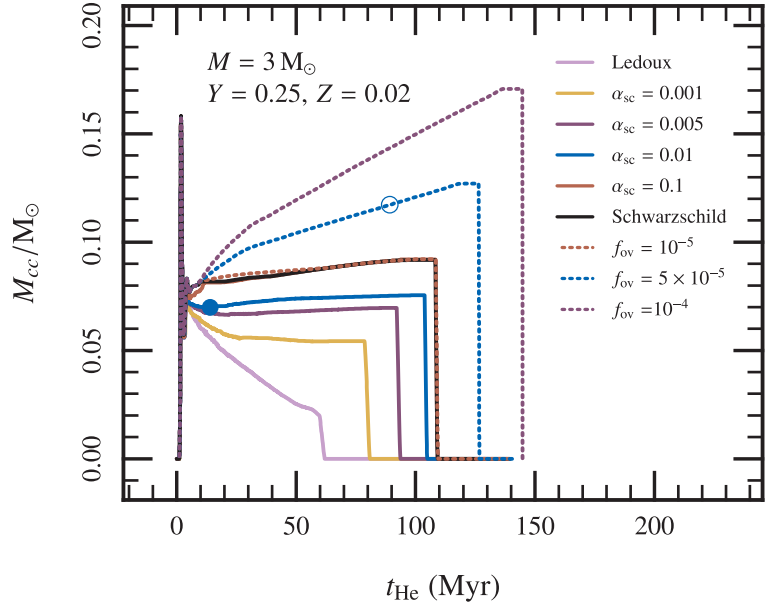

Figure 15. History of convective core extent during the core helium burning phase for a non-rotating $3 M_{\odot}$ star with various mixing options, as in Figure 13. Time is measured relative to the onset of the convective core burning. Efficient semiconvection $\left(\alpha_{\mathrm{sc}}=0.01\right)$ and inefficient overshooting $\left(f_{\mathrm{ov}}=10^{-5}\right)$ coincide with the pure Schwarzschild model. The filled (open) circle indicates the time for which we display a profile detailing semiconvection (overshooting) in Figure 11.

(A color version of this figure is available in the online journal.)

tion of overshoot. This instability is not seen in overshoot during hydrogen burning and has yet to be studied in detail.

\section{EVOLUTION BEYOND THE MAIN SEQUENCE AND WHITE DWARFS}

Extending the verification of Paper I, we now compare to other available codes for intermediate-mass stars, $3-8 M_{\odot}$. We describe the techniques used by MESAstar to evolve stars through the AGB phase to the WD cooling sequence. We also demonstrate how MESAstar incorporates compressional heating from accretion.

\subsection{Code Comparisons during Helium Core Burning}

We start by comparing the results of MESAstar to those from the Dartmouth Stellar Evolution Program (DSEP; Dotter et al. 2008) for stars with $M=3-8 M_{\odot}$. In both cases, the models were evolved from the PMS to the depletion of helium in their cores. For completeness, the MESAstar models were further evolved to the occurrence of the first helium thermal pulse.

All models have an initial composition $Y=0.272, Z=0.02$, and no mass loss or rotation was included. The boundaries of mixing zones are determined by the Schwarzschild criterion with $\alpha_{\mathrm{MLT}}=2$. In order to compare the codes, we do not allow overshooting or semiconvection. We adopt the Kunz et al. (2002) rate for ${ }^{12} \mathrm{C}(\alpha, \gamma){ }^{16} \mathrm{O}$ and the Imbriani et al. (2004) rate for ${ }^{14} \mathrm{~N}(p, \gamma){ }^{15} \mathrm{O}$; for all other rates we use the NACRE compilation (Angulo et al. 1999). We use the OPAL Type 2 opacity tables (Iglesias \& Rogers 1993) to account for the carbon- and oxygenenhanced opacities during helium burning.

The resulting tracks in the H-R diagram of Figure 16 and the evolution in the $T_{\mathrm{c}}-\rho_{\mathrm{c}}$ plane of Figure 17 show excellent agreement between the codes. Figures 18 and 19 show the hydrogen-burning luminosity, the helium-burning luminosity, and the extent of the convective core during convective helium core burning for a $4 M_{\odot}$ model (Figure 18) and a $6 M_{\odot}$ model (Figure 19). Table 3 gives a summary of the core hydrogen burning lifetime, the core helium burning lifetime, the final extent of the convective core during central helium burning, and 
Table 3

Properties of the 3-8 $M_{\odot}$ Evolution (Masses in Solar Units)

\begin{tabular}{|c|c|c|c|c|c|c|c|c|c|c|c|}
\hline \multirow[b]{2}{*}{$M / M_{\odot}$} & \multicolumn{7}{|c|}{ MESA } & \multicolumn{4}{|c|}{ DSEP } \\
\hline & $\Delta t_{\mathrm{H}^{\mathrm{a}}}$ & $M_{\mathrm{cc}}^{\operatorname{maxb}}$ & $M_{\text {core }}^{\mathrm{He}}$ & $\Delta t_{\mathrm{He}}{ }^{\mathrm{d}}$ & $M_{\mathrm{cc}}^{f_{\mathrm{e}}}$ & $X_{\mathrm{C}}^{\mathrm{f}}$ & $M_{\text {core }}^{\mathrm{CO} g}$ & $\Delta t_{\mathrm{H}}{ }^{\mathrm{a}}$ & $\Delta t_{\mathrm{He}}{ }^{\mathrm{d}}$ & $M_{\mathrm{cc}}^{f_{\mathrm{e}}}$ & $X_{\mathrm{C}}{ }^{\mathrm{f}}$ \\
\hline 3.0 & 320.6 & 0.69 & 0.36 & 83.59 & 0.097 & 0.426 & 0.466 & 312.0 & 80.81 & 0.098 & 0.456 \\
\hline 4.0 & 152.7 & 1.01 & 0.47 & 29.78 & 0.149 & 0.490 & 0.667 & 147.3 & 28.91 & 0.153 & 0.516 \\
\hline 5.0 & 85.61 & 1.34 & 0.59 & 15.52 & 0.214 & 0.511 & 0.827 & 84.75 & 15.19 & 0.210 & 0.507 \\
\hline 6.0 & 55.98 & 1.68 & 0.72 & 9.62 & 0.288 & 0.514 & 0.870 & 55.41 & 9.61 & 0.289 & 0.505 \\
\hline 7.0 & 39.91 & 2.03 & 0.86 & 6.51 & 0.375 & 0.511 & 0.915 & 39.69 & 6.79 & 0.401 & 0.454 \\
\hline 8.0 & 30.42 & 2.40 & 1.02 & 4.67 & 0.480 & 0.504 & 0.966 & 30.26 & 4.71 & 0.482 & 0.515 \\
\hline
\end{tabular}

Notes. Selected quantities are also shown from DSEP for comparison.

${ }^{a}$ Central $\mathrm{H}$ burning lifetime (Myr).

b Maximum extent of the convective core during core $\mathrm{H}$ burning.

${ }^{\mathrm{c}}$ Mass of the He core before central He ignition.

${ }^{\mathrm{d}}$ Central He burning lifetime (Myr).

e Stable final extent of the Schwarzschild convective core during core He burning.

${ }^{\mathrm{f}}$ Central mass fraction of ${ }^{12} \mathrm{C}$ at the end of core He burning.

g Mass of the $\mathrm{C} / \mathrm{O}$ core at the time of the first thermal pulse.

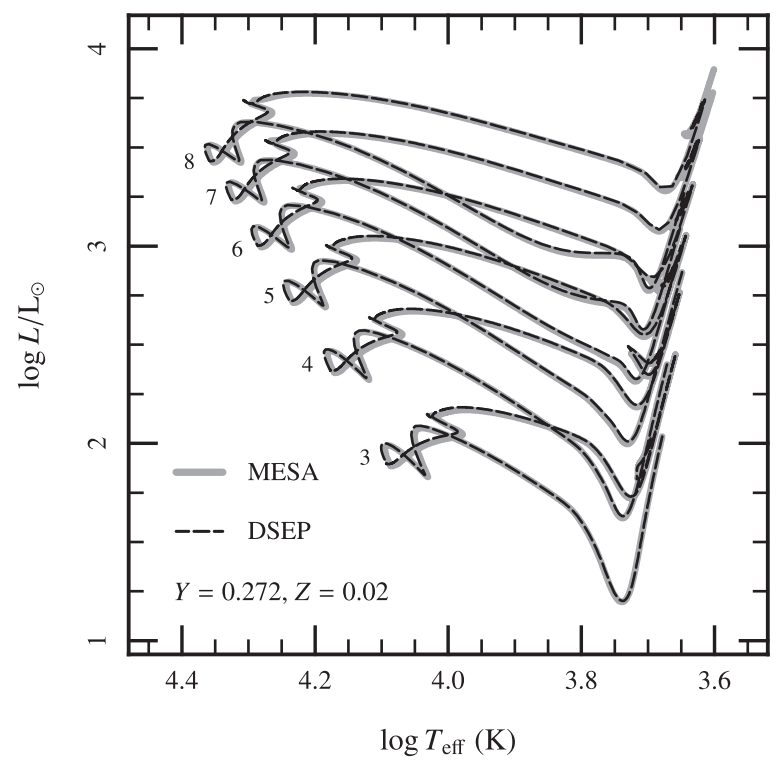

Figure 16. Hertzsprung-Russell diagram for evolution of 3-8 $M_{\odot}$ stars from the pre-main sequence through core helium depletion. Models are from MESA (thick gray lines) and DSEP (dashed black lines). Each curve is labeled with its corresponding initial mass in solar units.

the final carbon mass fraction $X_{\mathrm{C}}$ in the core for each model. For the MESA models, we also show the maximum extent of the convective core during central hydrogen burning, the mass of the helium core before helium ignition, and the mass of the $\mathrm{C} / \mathrm{O}$ core at the time of the first helium thermal pulse.

We close with an additional comparison of the helium core burning phase of a $M=3 M_{\odot}, Z=0.02$ model computed by MESA to that of Straniero et al. (2003). Both models were evolved using the Kunz et al. (2002) rate for ${ }^{12} \mathrm{C}(\alpha, \gamma){ }^{16} \mathrm{O}$. The results for MESAstar are a helium core burning lifetime of 83.6 Myr and final $\mathrm{C} / \mathrm{O}$ mass fractions of $X_{\mathrm{C}}=0.43$, $X_{\mathrm{O}}=0.55$; Straniero et al. (2003) find a lifetime of $88 \mathrm{Myr}$ and $X_{\mathrm{C}}=0.42, X_{\mathrm{O}}=0.56$.

\subsection{Making and Cooling White Dwarfs}

In the previous section, we discussed the evolution of 3-8 $M_{\odot}$ stars up to the occurrence of the first He thermal pulse. In Paper I we showed detailed comparisons of the evolution of a $2 M_{\odot}$

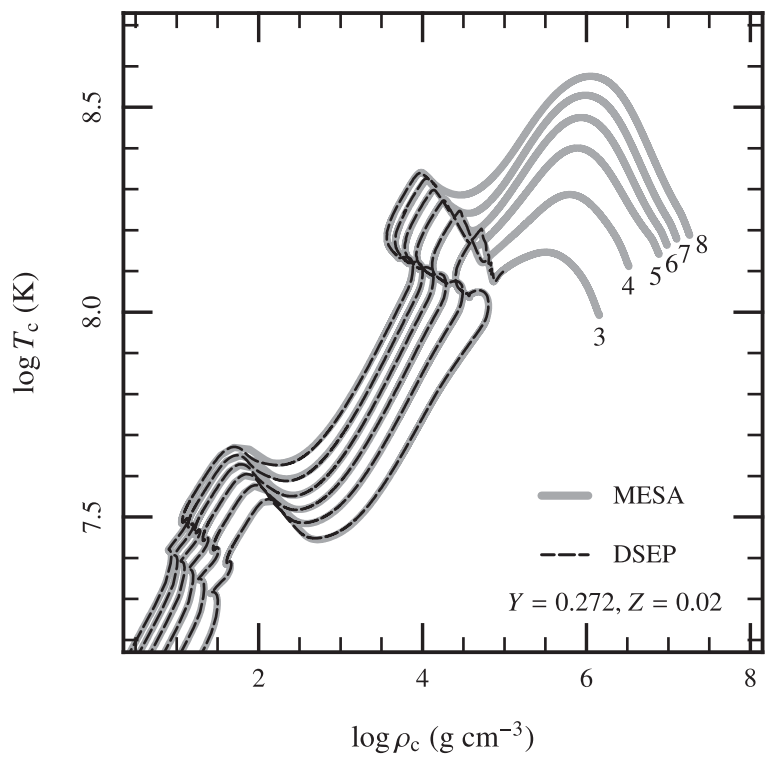

Figure 17. Same as Figure 16, but in the $T_{\mathrm{c}}-\rho_{\mathrm{c}}$ plane. The MESA models (thick gray lines) are evolved until the occurrence of the first thermal pulse.

star to the EVOL code (Herwig 2004), exhibiting the ability of MESAstar to calculate multiple helium shell pulses. We now illustrate the final evolution of intermediate-mass stars, and how to construct WDs by using winds.

We evolve $3 M_{\odot}, 5 M_{\odot}$, and $7 M_{\odot}$ stars from the ZAMS using the test suite case make_co_wd. This makes use of RGB mass loss following Reimers (1975) with an efficiency parameter $\eta=0.5$ and AGB mass loss following Bloecker (1995) using $\eta=0.1$ until the occurrence of the first helium shell flash. At that time, an increased Bloecker $\eta=5$ is adopted to allow only a small number of thermal pulses before the wind mass loss eliminates the envelope. Such intervention allows MESAstar to make a high-mass WD. To avoid shortening of time steps due to radiation-dominated envelopes, these cases also use the MLT++ capability described in Section 7.2.

Figure 20 shows the resulting tracks on the H-R diagram. The $3 M_{\odot}$ star underwent eight thermal pulses after the enhancement of Bloecker winds, while the $5 M_{\odot}$ and $7 M_{\odot}$ stars lost their envelopes so quickly that thermal pulses were immediately halted. The $5 M_{\odot}$ star ended up as an $M=0.844 M_{\odot} \mathrm{C} / \mathrm{O}$ 


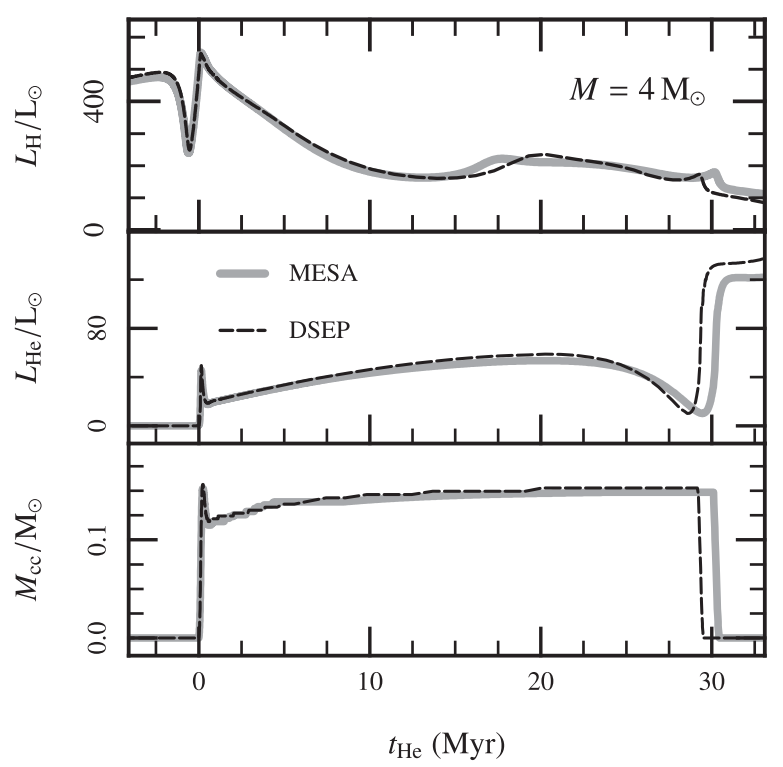

Figure 18. History of hydrogen burning luminosity (top), helium-burning luminosity (center), and convective core extent (bottom) during the core helium burning phase for the $4 M_{\odot}$ models. Time is measured relative to the onset of the convective core.

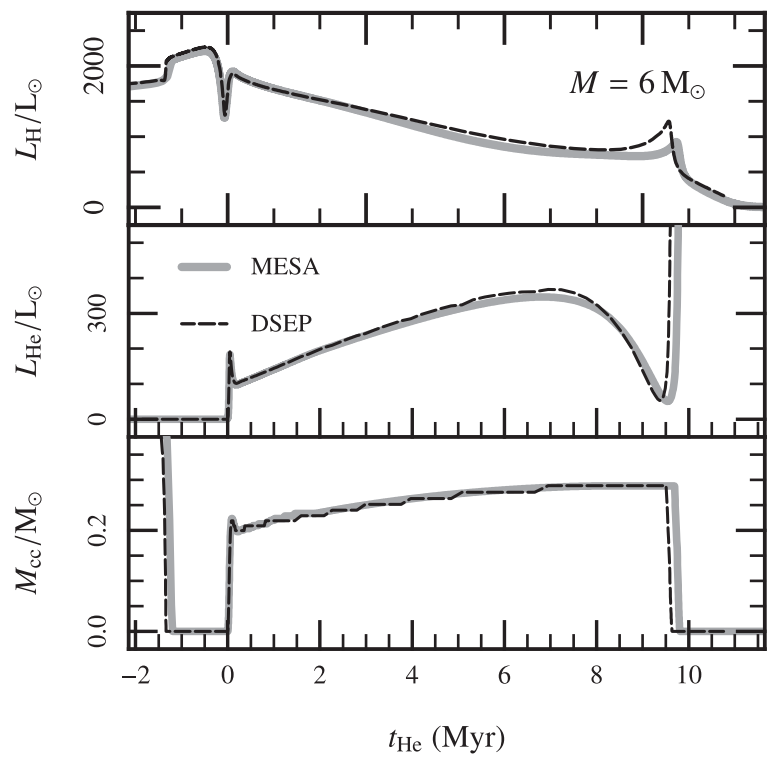

Figure 19. History of hydrogen burning luminosity (top), helium-burning luminosity (center), and convective core extent (bottom) during the core helium burning phase for the $6 M_{\odot}$ models. Time is measured relative to the onset of the convective core.

WD with a helium shell of thickness $M_{\mathrm{He}}=0.009 M_{\odot}$ and a hydrogen envelope of $M_{\mathrm{H}}=2.3 \times 10^{-5} M_{\odot}$. Note that the $\mathrm{C} / \mathrm{O}$ WD mass is only slightly larger than the $\mathrm{C} / \mathrm{O}$ mass at the first thermal pulse $\left(0.827 M_{\odot}\right)$ reported in Table 3 .

After removal of the envelope, the evolution of the WD is continued through its cooling phase past solidification. We include gravitational settling and chemical diffusion of the outermost layers. Figure 21 shows $T-\rho$ profiles taken at various effective temperatures during the cooling of the $M=0.844 M_{\odot}$ C/O WD made from the $5 M_{\odot}$ star. The growing depth of the convection zone is shown by the dashed line, and the open circles designate the $\mathrm{H} / \mathrm{He}$ transition, while the filled circles denote the $\mathrm{He} / \mathrm{CO}$ transition. Figure 22 illustrates the resulting $L-T_{\mathrm{c}}$ relation as these models cool.

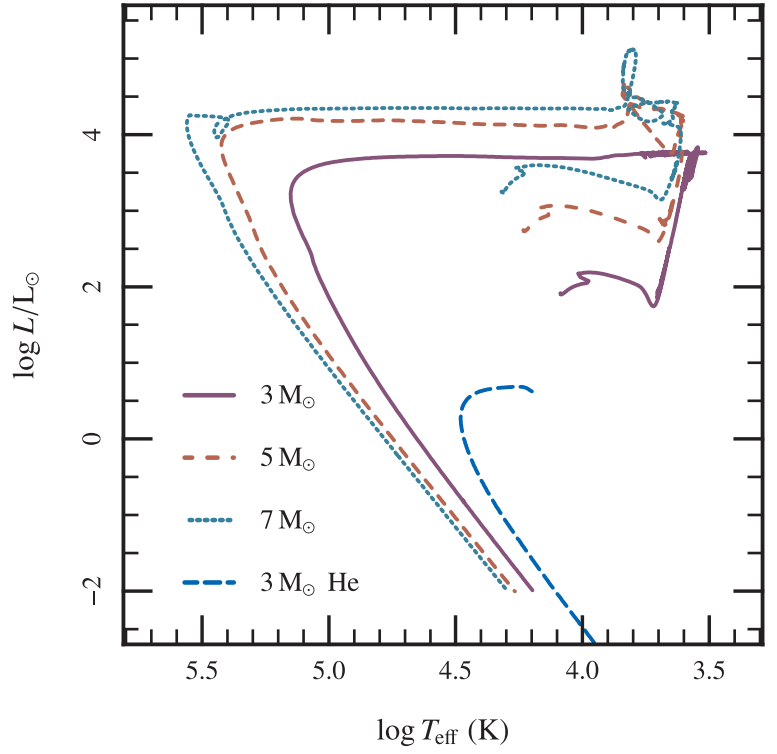

Figure 20. Evolution of 3,5 and $7 M_{\odot}$ models from zero-age main sequence to cooling white dwarfs. A Bloecker mass loss strips the stars of their envelopes on the thermally pulsing AGB to make the three $\mathrm{C} / \mathrm{O}$ white dwarfs. The single $0.32 M_{\odot}$ He white dwarf was made with mass loss after the hydrogen main sequence for the $3 M_{\odot}$ model was completed.

(A color version of this figure is available in the online journal.)

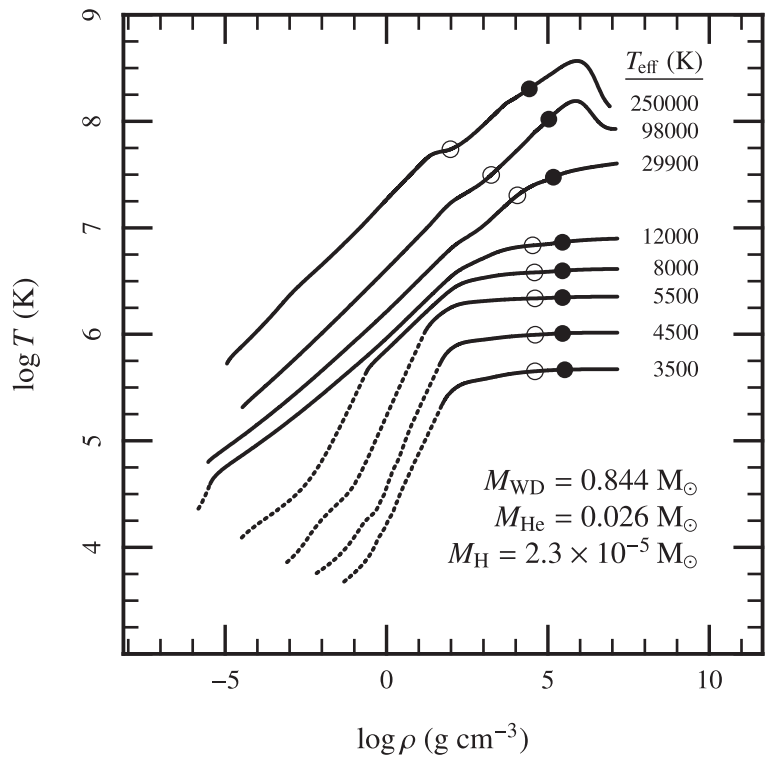

Figure 21. Profiles in $\log T-\log \rho$ space of the cooling $0.844 M_{\odot} \mathrm{C} / \mathrm{O}$ white dwarf evolved from a $5 M_{\odot}$ progenitor. Each model is labeled on the right by $T_{\text {eff }}$. The outermost point of the model is at $\tau=25$. Dotted curves denote convective regions. Going toward the interior, open circles designate the transition into the helium-rich shell, and filled circles designate the transition into the $\mathrm{C} / \mathrm{O}$ core.

The test suite case wd_diffusion uses the implementation of diffusion described in Paper I to evolve a WD of mass $0.535 M_{\odot}$ until the $M_{\mathrm{H}}=5.9 \times 10^{-5} M_{\odot}$ hydrogen layer and the $M_{\mathrm{He}}=1.0 \times 10^{-2} M_{\odot}$ helium layer approach diffusive equilibrium. At this point, the WD has an effective temperature of $T_{\text {eff }} \approx 5000 \mathrm{~K}$. We show the resulting abundance profiles in Figure 23, and, for comparison, the abundance profiles derived from the analytic form for diffusive equilibrium (Equation (22) of Althaus et al. 2003). This formula is obtained by integrating Equation (A.5) of Arcoragi \& Fontaine (1980) and assuming an ideal gas EOS and complete ionization of both species. 


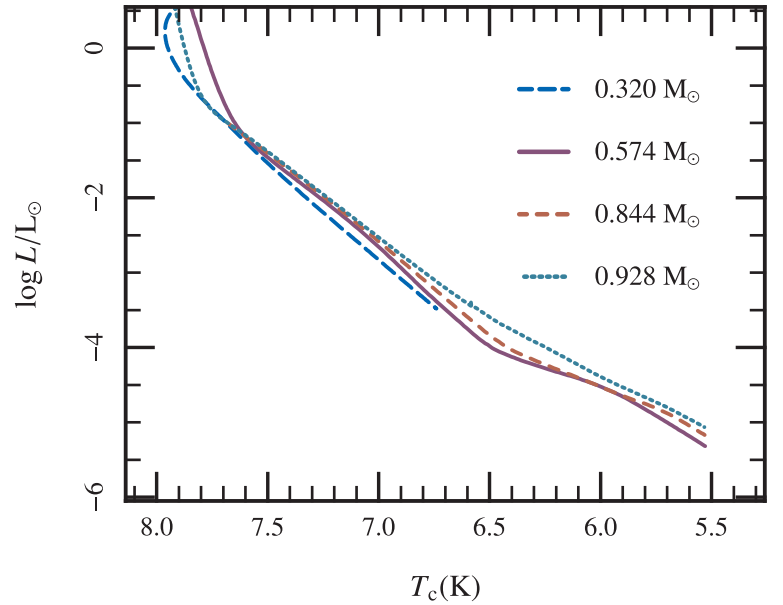

Figure 22. Surface luminosity as a function of central temperature for the cooling $0.32,0.574,0.844$, and $0.928 M_{\odot}$ WDs evolved from 3, 5, and $7 M_{\odot}$ progenitors.

(A color version of this figure is available in the online journal.)

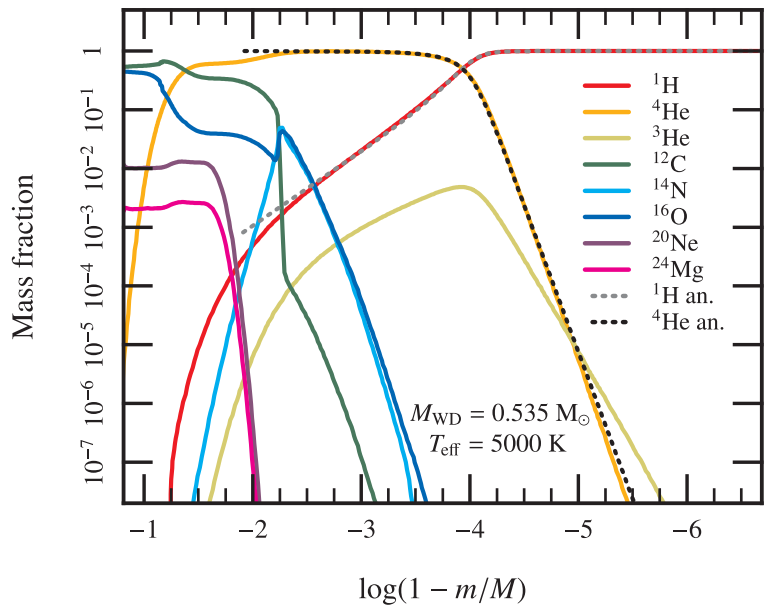

Figure 23. Comparison of time-dependent diffusion calculations for a $M=$ $0.535 M_{\odot}$ WD with $M_{\mathrm{H}}=5.9 \times 10^{-5} M_{\odot}$ and $M_{\mathrm{He}}=1.0 \times 10^{-2} M_{\odot}$ with MESAstar (solid lines) to those assuming diffusive equilibrium and an ideal gas equation of state (dashed lines).

(A color version of this figure is available in the online journal.)

The specific treatment of convection can also impact WD evolution. In Paper I, MESA used the Cox \& Giuli (1968) prescription for convection as its default convective MLT, with the optional extension of Henyey et al. (1965). Since Paper I, we have added support for the formulations of Böhm-Vitense (1958), Böhm \& Cassinelli (1971), and Mihalas (1978). In particular, the Böhm \& Cassinelli prescription, often referred to as "ML2," is frequently employed in WD studies (e.g., Bergeron et al. 1995). In Figure 24 we show a comparison of the Brunt-Väisälä frequency calculated with MESA to that using the Warsaw envelope code (Paczyński 1969, 1970; Pamyatnykh 1999), assuming the ML2 prescription. This is the same WD as in Figure 23, but now at a lower $T_{\text {eff }}=11,354 \mathrm{~K}$. To more accurately integrate these opaque but thin layers, we reduce $\tau$ at the boundary of the model by a factor of 1000 from its photospheric value of $2 / 3$. This calculation is a sensitive test of the envelope integrations because $N^{2}$ is a derivative of the envelope structure. The two codes give indistinguishable results for this case and all other cases that we have calculated.

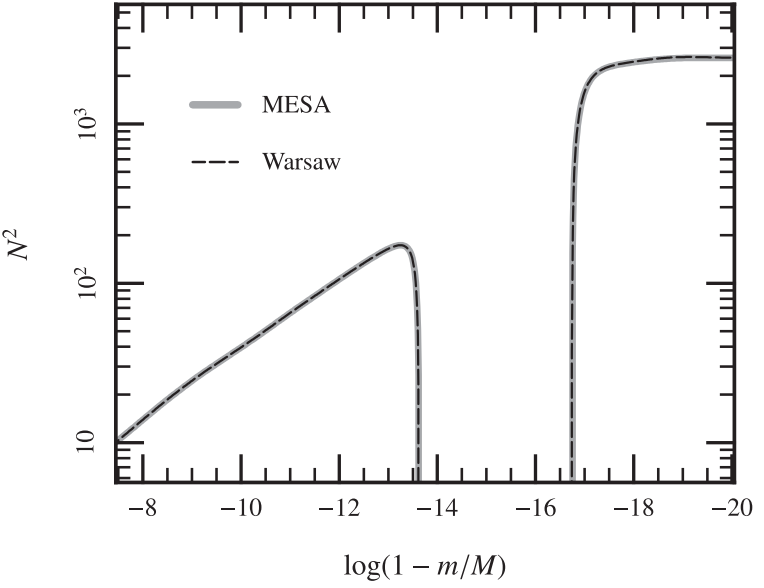

Figure 24. Comparison of the Brunt-Väisälä frequency calculated with MESA (solid gray line) to that using the Warsaw envelope code pig35.f (dashed line) for the same WD in Figure 23, but at a cooler $T_{\text {eff }}=11,354 \mathrm{~K}$.

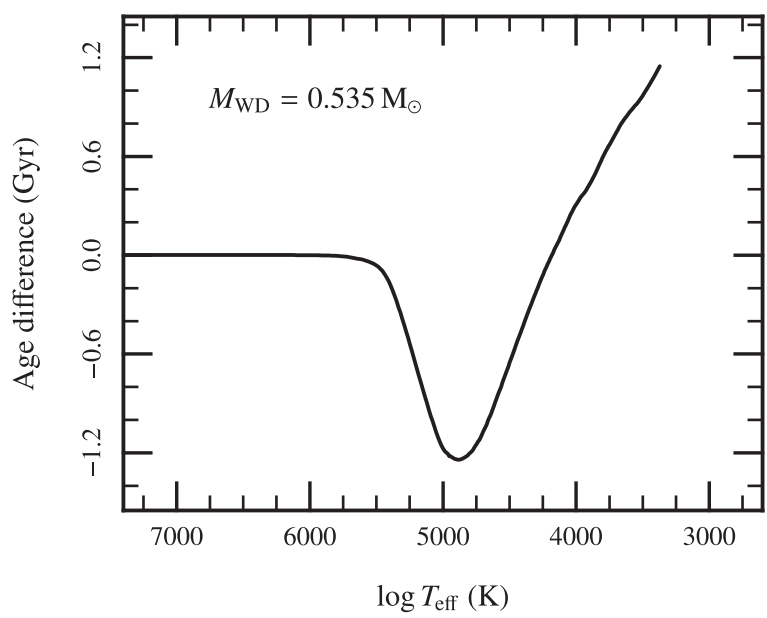

Figure 25. Age difference (non-gray minus gray) in Gyr as a function of $T_{\text {eff }}$.

MESA now includes atmospheric tables based on the non-gray model atmospheres for hydrogen-atmosphere WDs (Rohrmann 2001; Rohrmann et al. 2012), spanning the following range of parameters: $2000 \mathrm{~K} \leqslant T_{\text {eff }} \leqslant 40,000 \mathrm{~K}$ and $5.5 \leqslant \log g \leqslant 9.5$. Such an approach is necessary at $T_{\text {eff }} \lesssim 6000 \mathrm{~K}$, where WDs develop deeper convection zones. When the convection zone comes in contact with the degenerate, nearly isothermal core, energy is able to flow out of the core much more efficiently. The use of non-gray atmosphere models results in shallower convection zones, so this convective coupling of the core and envelope is delayed. For reliable cooling ages, we therefore recommend using non-gray atmospheres when $T_{\text {eff }} \lesssim 6000 \mathrm{~K}$. Figure 25 demonstrates the impact of non-gray atmospheres with the $0.535 M_{\odot} \mathrm{WD}$, which was cooled with and without the non-gray atmosphere.

MESA currently treats crystallization by employing the Potekhin \& Chabrier (2010) EOS (PC EOS). The PC EOS is callable for arbitrary mixtures of chemical species and for densities with $\log \rho \geqslant 2.8$; it is applicable in the domains of non-degenerate and degenerate, non-relativistic and relativistic electrons, weakly and strongly coupled Coulomb liquids, and classical and quantum Coulomb crystals. The phase transition is first-order, so the PC EOS exhibits a latent heat between the solid and liquid phases, i.e., the entropy and internal energy both 
experience finite jumps. This energy is included in MESAstar models of cooling WDs through the gravitational source term in the energy equation,

$$
\epsilon_{\text {grav }} \equiv-T \frac{d S}{d t}
$$

This form for $\epsilon_{\text {grav }}$ replaces the default one (see Equation (16) below) in cells where $\Gamma \geqslant 160$ ( $\Gamma$ is the Coulomb coupling parameter), and is smoothly interpolated with the default form in cells where $130 \leqslant \Gamma<160$. The PC EOS uses the criterion $\Gamma=175$ to determine crystallization, but it is straightforward to include explicit crystallization curves for $\mathrm{C} / \mathrm{O}$ and other mixtures (e.g., Schneider et al. 2012; Medin \& Cumming 2010). For example, using the parameters of the model in Figure 23, the age difference at late times $\left(T_{\text {eff }}<3500 \mathrm{~K}\right)$ between a model with and without the latent heat of crystallization is $\approx 0.8 \mathrm{Gyr}$; a slightly larger value would be obtained using the phase diagram of Schneider et al. (2012). MESA does not currently treat phase separation of different chemical species upon crystallization.

Low mass WDs $\left(M \lesssim 0.4 M_{\odot}\right)$ with helium cores and hydrogen envelopes may be produced in binary systems when the envelope is stripped by the companion as the primary evolves up the giant branch (Iben 1991, and references therein). He-core WDs of mass $M \simeq 0.4-0.5 M_{\odot}$ may also be produced through strong RGB winds (D'Cruz et al. 1996), although we do not discuss this possibility further here.

Here we discuss the prescription for stripping the envelope used in the test case make_he_wd. The first step is to evolve a star, $M=3.0 M_{\odot}$ in this example, from the PMS until a He core of the correct size has been made. The remnant total mass is determined by the mass interior to where the $\mathrm{H}$ abundance has dropped below a preset value, for example, $X_{\mathrm{H}}=0.1$, moving in from the surface. Next, the routine relax_mass is used to remove mass from the model until it has the desired remnant mass. After the initial remnant has been constructed, diffusion can then be turned on to allow an outer $\mathrm{H}$ layer to form. After this stage, normal evolution of the WD occurs, as shown in Figures 20 and 22.

\subsection{Compressional Heating and Accretion}

Accretion onto stars occurs in many contexts and requires special treatment for the outermost layers added in each time step. In particular, a special evaluation of the $\epsilon_{\text {grav }}=-T d S / d t$ term is required for fluid parcels that were not present in the previous time step. Prior to addressing that subtlety, we restate (as discussed in Section 6.2 of Paper I) that MESAstar calculates $\epsilon_{\text {grav }}$ of Equation (15) in terms of the local thermodynamic variables $(T$ and $\rho$ ) used by MESA,

$$
\epsilon_{\text {grav }}=-C_{P} T\left[\left(1-\nabla_{\mathrm{ad}} \chi_{T}\right) \frac{d \ln T}{d t}-\nabla_{\mathrm{ad}} \chi_{\rho} \frac{d \ln \rho}{d t}\right] .
$$

MESAstar takes the quantities in this equation as provided by eos, and computes the Lagrangian time derivatives to find $\epsilon_{\text {grav }}$. MESAstar can alternatively work under the assumption that $P=P_{\text {gas }}+P_{\text {rad }}$, in which case MESAstar treats $P_{\text {gas }}$ rather than $\rho$ as its basic variable (see Appendix B.2 for a discussion). In that case,

$\epsilon_{\text {grav }}=-C_{P} T\left[\left(1-4 \nabla_{\mathrm{ad}} \frac{P_{\mathrm{rad}}}{P}\right) \frac{d \ln T}{d t}-\nabla_{\mathrm{ad}} \frac{P_{\mathrm{gas}}}{P} \frac{d \ln P_{\mathrm{gas}}}{d t}\right]$.
Either formulation can be used deep within the star, as long as the location is safely removed from any phase transition. Paper I described the validation of these formulations.

We now turn to the complication which arises when $\epsilon_{\text {grav }}$ needs to be evaluated in material that was not present in the previous time step. Defining the envelope mass coordinate $\Delta M \equiv M-m$, we need to resolve the entropy for $\Delta M<$ $\delta M=\dot{M} \delta t$, as the explicit Lagrangian time derivatives of Equations (16) and (17) cannot be numerically evaluated. Since there can be important physics that needs to be resolved for these mass shells for $\Delta M \ll \delta M$, an approximation must be derived that allows for accurate modeling of the star's outermost layers without having to result to a dramatic shortening of $\delta t$.

The luminosity $L_{\text {acc }}=G M \dot{M} / R$ from the accretion shock (or boundary layer) goes outward and does not determine the entropy of the material as it becomes part of the hydrostatically adjusting star. Rather, the entropy of the material at $\Delta M \ll \delta M$ is determined by the transport of $L$ (Nomoto \& Sugimoto 1977; Nomoto 1982; Townsley \& Bildsten 2004). Consider such an outermost layer, where there are two relevant timescales, the thermal time, $t_{\mathrm{th}}=C_{P} T \Delta M / L$, and the local accretion time, $t_{\mathrm{acc}}=\Delta M / M$. In nearly all relevant cases, the ratio $t_{\text {th }} / t_{\text {acc }}=C_{P} T \dot{M} / L \ll 1$; this implies that the fluid element adjusts its temperature to that needed to transport the stellar luminosity from deep within. This simplifies $\epsilon_{\text {grav }}$ in that part of the star (following Townsley \& Bildsten 2004) to

$$
\epsilon_{\text {grav }}=\frac{C_{P} T G m \dot{M}}{4 \pi r^{4} P}\left(\nabla_{\mathrm{ad}}-\nabla_{T}\right),
$$

enabling accurate modeling within MESAstar of nearly all fluid elements that become part of the star during each time step, many of which have envelope mass coordinates $\Delta M \ll \dot{M} \delta t$.

We give an explicit example of this thin-shell radiative calculation of $\epsilon_{\text {grav }}$ in a $\mathrm{C} / \mathrm{O} \mathrm{WD}$ accreting hydrogen-rich material and undergoing classical nova $(\mathrm{CN})$ cycles. We present two models accreting at rates of $\dot{M}=10^{-11} M_{\odot} \mathrm{yr}^{-1}$ and $10^{-10} M_{\odot} \mathrm{yr}^{-1}$. Both cases were evolved from a $0.6 M_{\odot}$ starting model with $T_{\mathrm{c}}=10^{7} \mathrm{~K}$ which had undergone a few flashes while accreting at $\dot{M}=10^{-11} M_{\odot} \mathrm{yr}^{-1}$. The accreted material has solar-like composition $X=0.70, Y=0.26$, and $Z=0.04$ where the metal mass fractions are taken from Lodders (2003).

Profiles of the envelope during the mass accumulation phase between $\mathrm{CN}$ outbursts for the two accretion rates are displayed in Figures 26 and 27. Each line represents a different time in the accumulation cycle up to the unstable ignition, when the hydrogen mass reaches $M_{\mathrm{H}}=M_{\mathrm{ign}}$. All material at pressures smaller than that shown by the open circle is new to the model in that time step (e.g., it has $\Delta M<\delta M$ ) and employs the modified $\epsilon_{\text {grav }}$ of Equation (18). This highlights the significance of this approximation as it allows MESAstar to calculate material properties at $\Delta M \sim 10^{-8} \delta M$. The solid points show where $\epsilon_{\text {grav }}$ switches to the explicit form employing the Lagrangian time derivatives, such as Equation (16).

The middle panel shows $\epsilon_{\text {grav }} P \propto \epsilon_{\text {grav }} \Delta M$, which reflects the contribution of $\epsilon_{\text {grav }}$ to the outward luminosity. The discontinuity of $\epsilon_{\text {grav }}$ at the solid point reflects the error associated with the abrupt transition in the calculational approach. The substantially larger luminosity of the early $\left(M_{\mathrm{H}} / M_{\mathrm{ign}}=0.22\right)$ stages is due to the ongoing transfer of heat from the previous outburst. The near-discontinuous drop in $\epsilon_{\text {grav }}$ occurs at the base of the hydrogen-rich envelope, and reflects the jump in composition from the accreted material to the nearly pure ${ }^{4} \mathrm{He}$ 


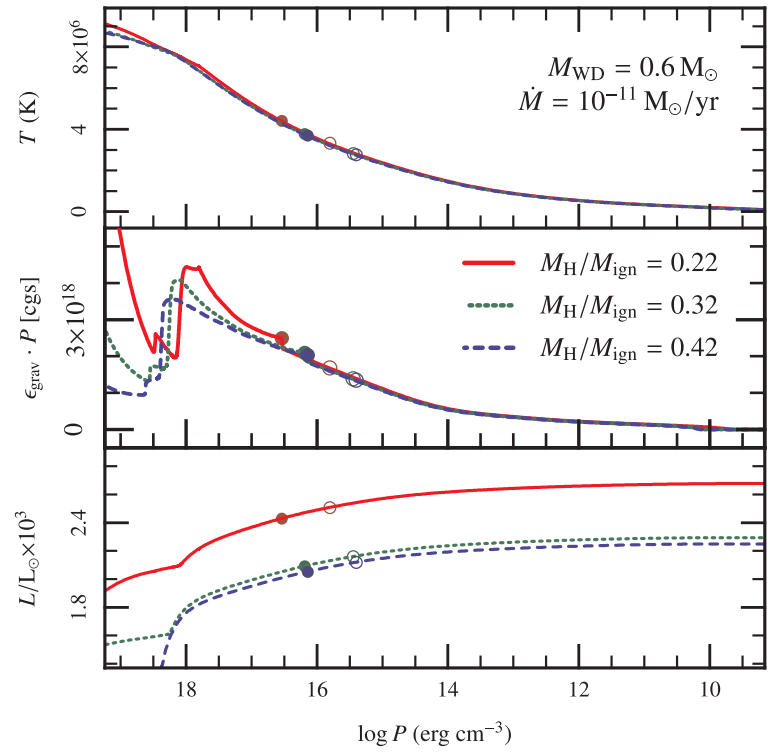

Figure 26. Envelope profiles as a function of pressure of the accreting white dwarf for three instants during the mass accumulation phase; $\dot{M}=$ $10^{-11} M_{\odot} \mathrm{yr}^{-1}$ model. The top panel shows temperature, the central panel shows the gravitational energy release rate, and the bottom shows the luminosity. Material to the right of the open circle is newly accreted. The code treats material to the right of the filled circle using the thin-shell radiative calculation of $\epsilon_{\text {grav }}$. (A color version of this figure is available in the online journal.)

layer. The expected amplitude of the jump in $\epsilon_{\text {grav }}$ depends on both the composition jump and the local degree of electron degeneracy (see Appendix B of Townsley \& Bildsten 2004 for a discussion).

\section{ROTATION}

A star's rotational energy is usually a small fraction of the gravitational energy: for the Sun it is $\sim 10^{-5}$ and for a $25 M_{\odot}$ star rotating with a typical equatorial velocity $v_{\mathrm{eq}}=200 \mathrm{~km} \mathrm{~s}^{-1}$ on the MS it is $\sim 0.04$. Therefore the effects on the stellar hydrostatic equilibrium are marginal, with the exception of stars close to critical rotation (see Section 6.4). Even in the case of a small perturbation to hydrostatic equilibrium, rotation induces a modification to the star's thermal equilibrium (von Zeipel 1924). Together with the emergence of rotationally induced dynamical and secular instabilities, this can significantly affect the evolution of stars (Maeder \& Meynet 2000b). Due to the destabilizing effect of increasing radiation pressure, rotation is particularly important in massive stars (see, e.g., Heger et al. 2000; Meynet \& Maeder 2000). Moreover, the final fate of a massive star depends chiefly on the relative importance of rotation during its evolution (e.g., Heger et al. 2000; Hirschi et al. 2004; Heger et al. 2005; Yoon et al. 2006; Woosley \& Heger 2006; Ekström et al. 2012; Georgy et al. 2012; Langer 2012).

Here we describe the implementation of rotation in MESAstar. We briefly discuss the modification to the stellar structure equations and the inclusion of rotationally- and magnetically induced mixing. Magnetic fields generated by differential rotation in radiative regions have been implemented following the work of Spruit (2002) and in the same fashion as in Petrovic et al. (2005) and Heger et al. (2005). Rotationally enhanced mass loss is also discussed.

We compare rotating massive-star models calculated with MESAstar to previous calculations performed with Kepler

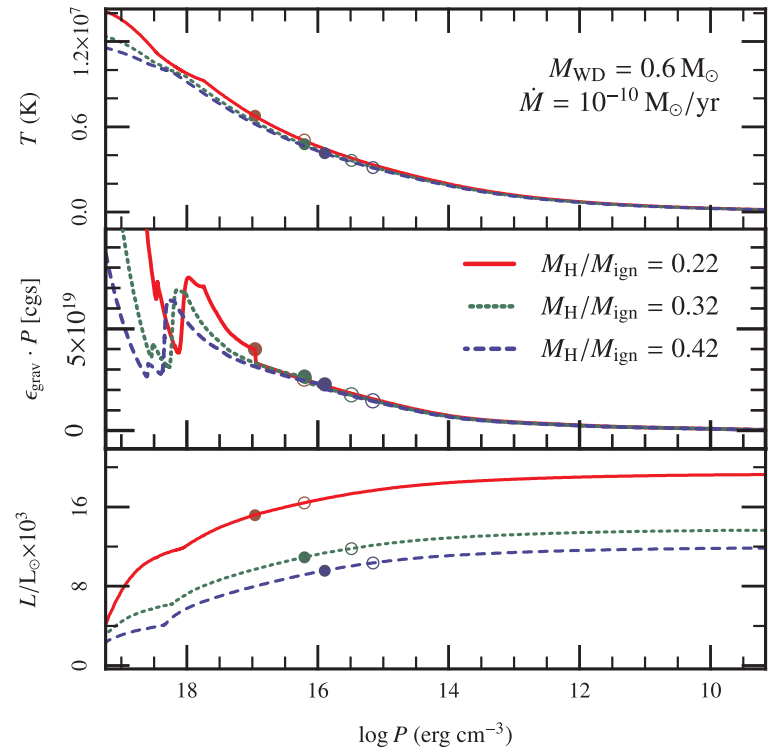

Figure 27. Same as Figure 26, but for model accreting $10^{-10} M_{\odot} \mathrm{yr}^{-1}$. (A color version of this figure is available in the online journal.)

(Heger et al. 2005). We also directly compare runs from MESAstar and STERN (Petrovic et al. 2005; Yoon \& Langer 2005; Brott et al. 2011). The purpose of these tests is to verify our implementation of rotation, which is derived from STERN. We do not compare to codes that have a different implementation of rotation (e.g., Hirschi et al. 2004; Ekström et al. 2012; Georgy et al. 2012; Potter et al. 2012b, 2012a), although beyond the scope of this paper, such comparisons are critical when coupled to observations of the effects of rotation in stars (e.g., Hunter et al. 2007; Evans et al. 2011) including asteroseismology (Beck et al. 2012; Mosser et al. 2012).

\subsection{Implementation of Shellular Rotation}

Stellar structure deviates from spherical symmetry in the presence of rotation. While the structure is inherently $3 \mathrm{D}$, it suffices to solve the stellar structure equations in one dimension if the angular velocity, $\omega$, is constant over isobars (the so-called shellular approximation; see, e.g., Meynet \& Maeder 1997). This is expected in the presence of strong anisotropic turbulence acting along isobars. In radiative regions such turbulence is a consequence of differential rotation (Zahn 1992) and efficiently erases gradients along isobars and enforces shellular rotation (Meynet \& Maeder 1997). Turbulence in the vertical direction (i.e., perpendicular to the isobars) is much weaker due to the stabilizing effect of stratification. In MESAstar we adopt the shellular approximation (Meynet \& Maeder 1997) and calculate the modification to the stellar equations due to centrifugal acceleration (Kippenhahn \& Thomas 1970; Endal \& Sofia 1976).

\subsubsection{Stellar Structure}

An isobar with volume $V_{\mathrm{P}}$ and surface area $S_{\mathrm{P}}$ deviates from spherical symmetry in the presence of rotation. However one can retain a $1 \mathrm{D}$ approximation by redefining the radius coordinate as the radius of a sphere containing the same volume $V_{\mathrm{P}}=4 \pi r_{\mathrm{P}}{ }^{3} / 3$, allowing an equation of continuity in the usual form

$$
\left(\frac{\partial m_{\mathrm{P}}}{\partial r_{\mathrm{P}}}\right)_{t}=4 \pi r_{\mathrm{P}}^{2} \rho,
$$


with $\rho$ being the density and $m_{\mathrm{P}}$ the mass enclosed by $S_{\mathrm{P}}$. The energy equation also retains its usual, non-rotating form

$$
\left(\frac{\partial L_{\mathrm{P}}}{\partial m_{\mathrm{P}}}\right)_{t}=\epsilon_{\mathrm{nuc}}-\epsilon_{v}+\epsilon_{\mathrm{grav}}
$$

where $L_{\mathrm{P}}$ is the rate of energy flow through the equipotential surface $S_{\mathrm{P}}$. Then the next step is to define mean values for the quantities varying on isobars,

$$
\langle\cdot\rangle \equiv \frac{1}{S_{\mathrm{P}}} \oint_{S_{\mathrm{P}}} \cdot d \sigma
$$

where $d \sigma$ is an isobaric surface area element. The equation of momentum balance can be written as

$$
\left(\frac{\partial P}{\partial m_{\mathrm{P}}}\right)_{t}=-\frac{G m_{\mathrm{P}}}{4 \pi r_{\mathrm{P}}{ }^{4}} f_{\mathrm{P}}-\frac{1}{4 \pi r_{\mathrm{P}}^{2}}\left(\frac{\partial^{2} r_{\mathrm{P}}}{\partial t^{2}}\right)_{m_{\mathrm{P}}},
$$

where $P$ is the pressure, $G$ is the gravitational constant and $t$ the time. The last term in the equation is the inertia term. Rotation enters the momentum equation through the quantity $f_{\mathrm{P}}$

$$
f_{\mathrm{P}} \equiv \frac{4 \pi r_{\mathrm{P}}^{4}}{G m_{\mathrm{P}} S_{\mathrm{P}}}\left\langle g^{-1}\right\rangle^{-1},
$$

where $g \equiv|g|$, with $g$ the effective gravitational acceleration ( $g$ is normal to $S_{\mathrm{P}}$ ). Then the radiative temperature gradient becomes

$$
\left(\frac{\partial \ln T}{\partial \ln P}\right)_{t}=\frac{3 \kappa}{16 \pi a c G} \frac{P}{T^{4}} \frac{L_{\mathrm{P}}}{m_{\mathrm{P}}} \frac{f_{\mathrm{T}}}{f_{\mathrm{P}}}\left[1+\frac{r_{\mathrm{P}}^{2}}{G m_{\mathrm{P}} f_{\mathrm{P}}}\left(\frac{\partial^{2} r_{\mathrm{P}}}{\partial t^{2}}\right)_{m_{\mathrm{P}}}\right]^{-1},
$$

with $a$ the radiation constant, $\kappa$ the opacity, $T$ the temperature, and $L_{\mathrm{P}}$ the energy flux through $S_{\mathrm{P}}$. The last factor on the righthand side accounts for inertia, and

$$
f_{\mathrm{T}} \equiv\left(\frac{4 \pi r_{\mathrm{P}}^{2}}{S_{\mathrm{P}}}\right)^{2}\left(\langle g\rangle\left\langle g^{-1}\right\rangle\right)^{-1}
$$

In rotating models the values of $f_{\mathrm{T}}$ and $f_{\mathrm{P}}$ differ from 1 mostly in the outer stellar layers. Limits to the minimum values of $f_{\mathrm{T}}$ and $f_{\mathrm{P}}$ are set in the code (default values are 0.95 and 0.75 , respectively). This prevents numerical instabilities in models approaching critical rotation $\left(\Omega / \Omega_{\text {crit }}=1\right.$, see Section 6.4). In such cases the outer layers greatly deviates from spherical symmetry and the results from 1D calculations should be considered particularly uncertain.

\subsubsection{Mixing and Angular Momentum Transport}

Transport of angular momentum and chemicals due to rotationally induced instabilities is implemented in a diffusion approximation (e.g., Endal \& Sofia 1978; Pinsonneault et al. 1989; Heger et al. 2000). This choice has also been adopted by other stellar evolution codes (e.g., Kepler, Heger et al. 2000; STERN, Yoon \& Langer 2005). We stress that this is not the only possibility, and other groups have implemented a diffusion-advection approach (e.g., GENEVA, Eggenberger et al. 2008; RoSE, Potter et al. 2012b). The RoSE code can switch between the two different implementations. The two approaches are equivalent for the transport of chemicals. Potentially large differences can arise, however, for the transport of angular momentum. A detailed description of the advection-diffusion equation for angular momentum is given in Zahn (1992) and Maeder \& Zahn (1998).

In MESAstar the turbulent viscosity $v$ is determined as the sum of the diffusion coefficients for convection, semiconvection, and rotationally induced instabilities. In convective regions, the very large diffusion coefficient implies that the rotation law is not far from solid body. This is a very common assumption in stellar evolution codes (e.g., Pinsonneault et al. 1989; Heger et al. 2000; Eggenberger et al. 2008); note however that helioseismology has clearly shown this is not the case for the solar convection zone (e.g., Brown et al. 1989; Thompson et al. 1996; Schou et al. 1998). MESAstar calculates diffusion coefficients for five different rotationally induced mixing processes: dynamical shear instability, Solberg-Høiland instability, secular shear instability, Eddington-Sweet circulation, and the Goldreich-Schubert-Fricke instability. See Heger et al. (2000) for a detailed description of the physics of the different instabilities and the calculation of the respective diffusion coefficients. These enter the angular momentum and abundance diffusion equations that are solved at each time step (see Appendix B.6).

\subsection{Internal Magnetic Fields}

It has been suggested that differential rotation in the radiative layers of a star can amplify a seed magnetic field. Such a dynamo process has been proposed by Spruit (2002, Spruit-Tayler dynamo); a theoretical debate on this is still ongoing (Braithwaite 2006; Zahn et al. 2007; Denissenkov \& Pinsonneault 2007). From an observational point of view, pure hydrodynamic models fail to predict the solar core rotation (e.g., Pinsonneault et al. 1989), with the exception of models that include transport of angular momentum by gravity waves (Charbonnel \& Talon 2005). Models that include the Spruit-Tayler dynamo can reproduce the flat rotation profile of the Sun. Note however that these have difficulty explaining the core-envelope decoupling observed in low-mass, young cluster stars (Denissenkov et al. 2010). On the other hand, observations of the final spins of both WDs and neutron stars (Heger et al. 2005; Suijs et al. 2008) suggest that angular momentum transport with an efficiency similar to the torques provided by the Spruit-Tayler dynamo operates. Models that only include angular momentum transport through rotational instabilities do not produce the core-envelope ratio of angular velocity observed through the splitting of mixed modes in red giant stars (Eggenberger et al. 2012).

MESAstar accounts for transport by magnetic fields of angular momentum and chemicals due to the Spruit-Tayler dynamo. We refer to Spruit (2002) for a description of the physics of the dynamo loop and to Maeder \& Meynet (2003, 2004) and Heger et al. (2005) for a discussion of its inclusion in stellar evolution codes. We implement the Spruit-Tayler dynamo in MESAstar following Kepler (Heger et al. 2005) and STERN (Petrovic et al. 2005).

\subsection{Surface Magnetic Fields}

Rotating stars that have a significant outer convective zone can produce surface magnetic fields through a dynamo (see, e.g., Brandenburg \& Subramanian 2005 for a review on astrophysical dynamos). This is the case for low-mass MS stars below about $1.5 M_{\odot}$, and observationally the break in the rotation properties around this mass is attributed to the presence of magnetized stellar winds (e.g., Schatzman 1962; Kawaler 1988). Note that 
dynamo action in a subsurface convective layer is in principle also possible in early-type stars (Cantiello et al. 2009; Cantiello \& Braithwaite 2011). Surface magnetic fields can also be of fossil origin, as is usually discussed in the context of Ap stars (Braithwaite \& Spruit 2004). Whatever the origin of surface magnetic fields, these are expected to couple to the wind massloss and, if strong enough, produce magnetic braking (e.g., Weber \& Davis 1967; ud-Doula \& Owocki 2002; Meynet et al. 2011). Such magnetic braking has been directly observed in the case of the MS massive star $\sigma$-Ori E (Townsend et al. 2010). Here we do not include the physics of magnetic braking, as we only consider the evolution of stars without surface magnetic fields.

\subsection{Rotationally Enhanced Mass Loss}

We include the rotational modification to the wind mass loss rate (Friend \& Abbott 1986; Langer 1998; Heger \& Langer 1998; Maeder \& Meynet 2000a). Similar to other codes (e.g., Heger et al. 2000; Brott et al. 2011; Potter et al. 2012a), in MESAstar the stellar mass loss is enhanced as the rotation rate increases according to the prescription

$$
\dot{M}(\Omega)=\dot{M}(0)\left(\frac{1}{1-\Omega / \Omega_{\text {crit }}}\right)^{\xi},
$$

where $\Omega$ is the value of the surface angular velocity and $\Omega_{\text {crit }}$ is the critical angular velocity at the surface. This last quantity is defined as $\Omega_{\text {crit }}^{2}=\left(1-L / L_{\text {Edd }}\right) G M / R^{3}$, where $L_{\mathrm{Edd}}=4 \pi c G M / \kappa$ is calculated as a mass-weighted average in a user-specified optical depth range (default value $\tau \in[1-100]$ ). In MESAstar the default value for the exponent $\xi$ is 0.43 (Langer 1998). Other implementations of rotationally enhanced mass loss can be found in Maeder \& Meynet (2000a) and Georgy et al. (2011).

For stars approaching $\Omega / \Omega_{\text {crit }}=1$, the mass loss calculated using Equation (26) diverges. Notice that luminous stars can approach this limit without having to rotate very rapidly as $\Omega_{\text {crit }} \rightarrow 0$ when $L / L_{\text {Edd }} \rightarrow 1$. Following Yoon et al. (2010) we limit the mass loss timescale to the thermal timescale of the $\operatorname{star} \tau_{\mathrm{KH}}$

$$
\dot{M}=\min \left[\dot{M}(\Omega), f \frac{M}{\tau_{\mathrm{KH}}}\right],
$$

where $f$ is an efficiency factor of order unity (default value is $f=0.3$ ).

\subsection{Initial Models}

In all the rotating models presented in this paper, rotation is initialized by imposing a solid body rotation law on the zeroage main sequence (ZAMS; $L=L_{\text {nuc }}$ ). In these massive stars this is motivated by the presence of rotationally induced angular momentum transport during the PMS evolution. This alone is able to enforce a state of close-to-rigid rotation by the time the star reaches the ZAMS (Heger et al. 2000). Overall initial solid body rotation is a common choice in stellar evolution codes, but other rotational laws are certainly possible.

\subsection{Test Cases: $15 M_{\odot}$ and $25 M_{\odot}$}

As a first test we initialize a $15 M_{\odot}$ model with $Z=0.02$ and initial equatorial rotational velocity $v_{\mathrm{eq}}=200 \mathrm{~km} \mathrm{~s}^{-1}$ and run two calculations:
1. 15MAG includes the effects of rotation and Spruit-Tayler magnetic fields on both the transport of chemicals and angular momentum.

2. 15ROT includes only the effect of rotation on both the transport of chemicals and angular momentum;

The initial conditions have been calibrated to match as closely as possible the Kepler $15 M_{\odot}$ models (Heger et al. 2005). Moreover, we directly compare the MESAstar models with calculations from STERN (see, e.g., Yoon \& Langer 2005; Yoon et al. 2006). In particular we adopt a value of $f_{c}=1 / 30$ for the ratio of the turbulent viscosity to the diffusion coefficient and a value $f_{\mu}=0.1$ for the sensitivity to $\mu$ gradients (see Heger et al. 2000 for a discussion of these calibration parameters). The Ledoux criterion is used for the treatment of convective boundaries, together with semiconvection $\left(\alpha_{\mathrm{sc}}=1\right)$. We use $\alpha_{\text {MLT }}=1.6$, mass loss as in Yoon et al. (2006) with rotational enhancement as described in Section 6.4.

In Figure 28, we show the evolutionary track and the evolution of surface equatorial rotational velocity for the 15MAG model. Results of a similar calculation using STERN are shown as a dashed curve. The two results are in excellent agreement. Small differences in luminosity and lifetimes are not unexpected, as we have only matched the physics of rotation between the two calculations and not other ingredients. Values for the diffusion coefficients for rotationally induced mixing and magnetic torques during the MS of 15MAG are shown in Figure 29. The comparison reveals a very good agreement. Both stars are kept in solid-body rotation during the MS by the efficient transport of angular momentum provided by the Eddington-Sweet circulation and Spruit-Tayler magnetic fields.

The amplitude and location of the azimuthal $\left(B_{\phi}\right)$ and radial $\left(B_{r}\right)$ components of the magnetic fields during different phases of the evolution of 15MAG are shown in Figure 30. As expected, these fields are generated only in radiative regions of the star and $B_{\phi}>B_{r}$ (Spruit 2002). As the star evolves away from the MS its structure departs from solid-body rotation with the core rotating faster than the envelope. During this stage the role of magnetic fields is very important in transporting angular momentum from the core to the envelope. The effect can be seen in Figure 31, which shows the evolution of the internal specific angular momentum in models 15ROT and 15MAG. The presence of magnetic torques results in a dramatic spindown of the core of 15MAG with respect to 15ROT (see also Table 4). These results are in very good agreement with the ones obtained by STERN and Kepler.

As a second test, we now evolve a $25 M_{\odot}$ model (25MAG) with the same physics as in 15MAG. Figure 32 directly compare results with calculations performed with STERN. In Figure 33 we show a detailed comparison of the evolution of the internal specific angular momentum profile. We find a very good quantitative agreement between MESAstar and STERN down to He depletion in the core. The timescale for nuclear burning decreases substantially after He-burning and becomes shorter than the angular momentum transport timescale after $\mathrm{C}$ depletion. Thus only minor changes in the final angular momentum content of the stellar core are expected after this stage. Figure 34 shows the full evolution of the specific angular momentum profile of the MESAstar calculation from ZAMS to Si exhaustion.

\subsection{Rapidly Rotating Massive Stars}

MESAstar can calculate the evolution to core collapse of rapidly rotating massive stars. Rotational instabilities can be 

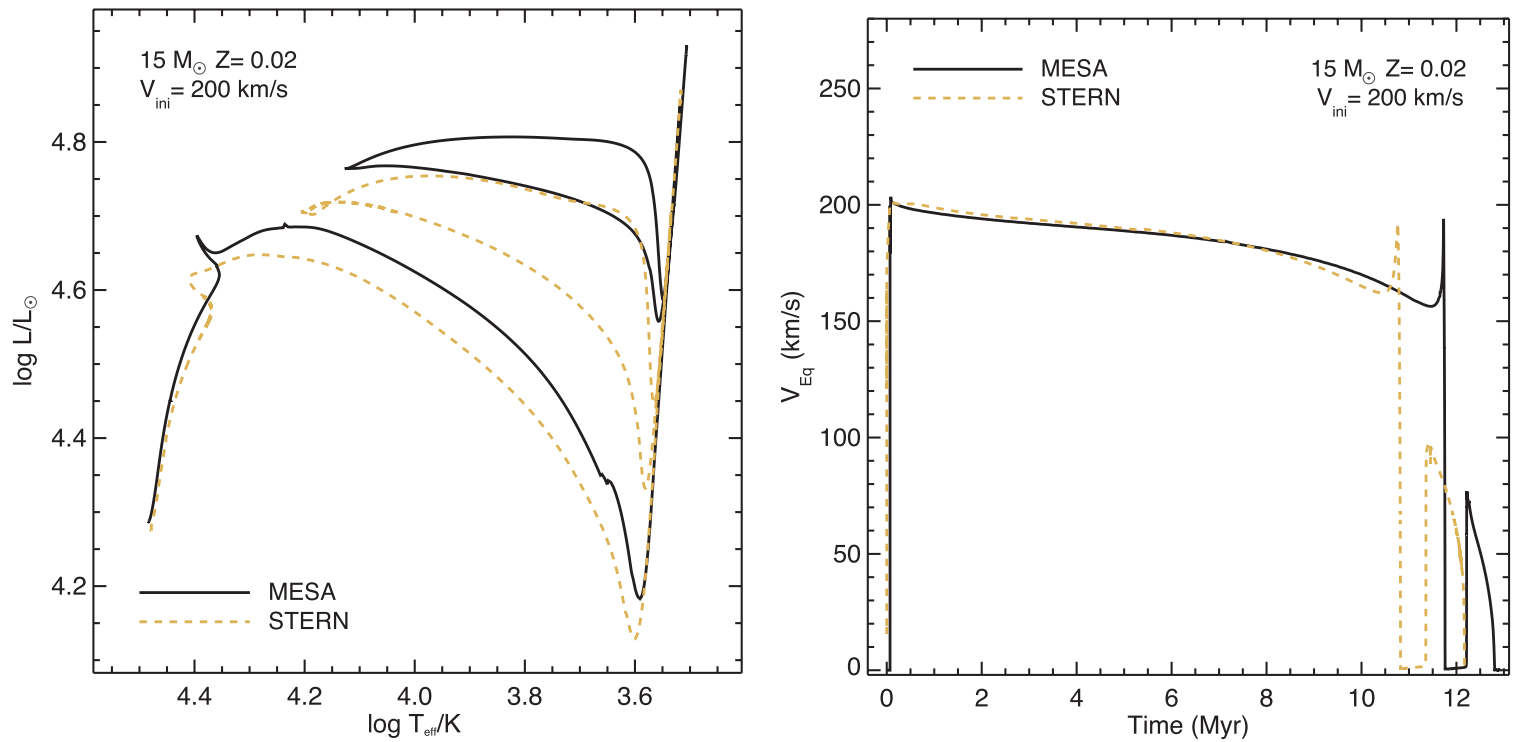

Figure 28. Comparison of evolutionary tracks (left) and equatorial rotational velocities (right) for a $15 M_{\odot}$ model with $Z=0.02$ rotating initially with $v_{\mathrm{eq}}=200 \mathrm{~km} \mathrm{~s}^{-1}$ (15MAG). The solid black line shows MESAstar results, and the dashed gold line shows the STERN calculations.

(A color version of this figure is available in the online journal.)
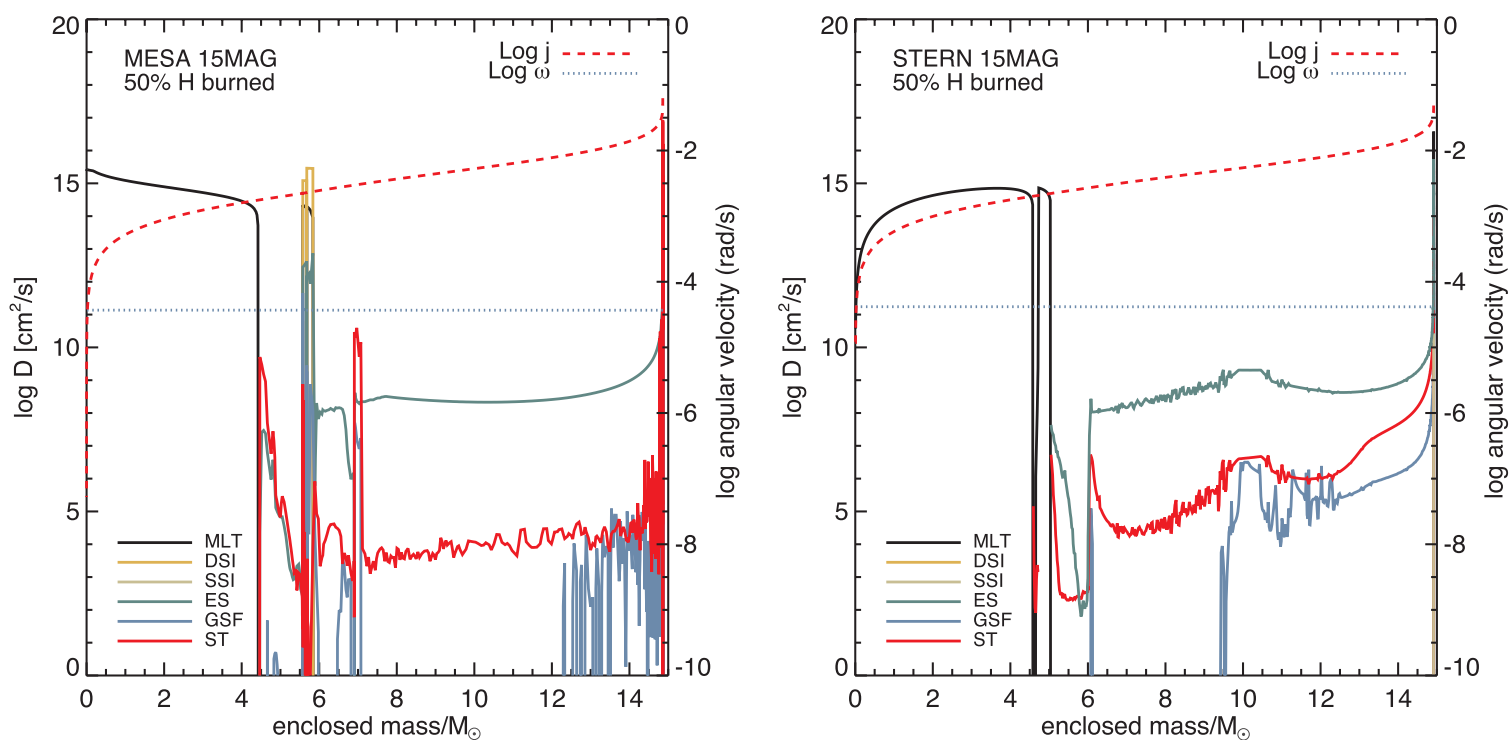

Figure 29. Same as Figure 28. As function of mass coordinate we plot the values of the diffusion coefficient for convection (MLT), Eddington-Sweet (ES) circulation, magnetic torques by dynamo generated fields (ST), dynamical shear (DSI), secular shear (SSI) and Goldreich-Schubert-Fricke (GSF) instability. Following STERN, we turn off the Solberg-Høiland (SH) instability for this comparison. This does not affect the results, as the diffusion coefficient for SH is usually smaller than the ones for ES and ST. The values of the specific angular momentum $j$ and the angular velocity $\omega$ are also plotted. Left panel shows the results using MESAstar, while the right panel shows analogous STERN calculations.

(A color version of this figure is available in the online journal.)

efficient enough to erase the compositional gradients built by nuclear burning. In such cases the model never develops a compositional stratification and remains almost completely mixed throughout its evolution (Maeder 1987b; Yoon \& Langer 2005; Woosley \& Heger 2006). This process leads to a bifurcation in the H-R diagram, with stars above a certain mass and rotation rate becoming more luminous and hotter. The threshold required for this bifurcation depends mostly on the initial mass of the star (Yoon et al. 2006). Metallicity also plays an important role, as angular momentum is lost through line-driven stellar winds, with mass-loss rates depending on the metallicity at the stellar surface (Vink et al. 2001). For the calculations in this section, we adopt the same mass-loss prescription as Yoon et al. (2006).
Figure 35 shows the evolution of two $16 M_{\odot}$ models at metallicity $Z=0.0002$ with rotation initialized at the ZAMS. One model is rotating very rapidly, with $v_{\mathrm{eq}}=450 \mathrm{~km} \mathrm{~s}^{-1}$ (corresponding to $\Omega / \Omega_{\text {crit }}=0.55$ and $J=3.23 \times 10^{52} \mathrm{erg} \mathrm{s}$ ), while the other rotates at $v_{\mathrm{eq}}=280 \mathrm{~km} \mathrm{~s}^{-1}$ (corresponding to $\Omega / \Omega_{\text {crit }}=0.39$ and $J=2.52 \times 10^{52} \mathrm{erg} \mathrm{s}$ ). The model with $\Omega / \Omega_{\text {crit }}=0.55$ avoids the core-envelope structure and becomes a compact Wolf-Rayet star. The absence of a red supergiant (RSG) phase eliminates the large magnetic torques from an extended envelope. The evolution of the internal profile of specific angular momentum in the two models clarify this point: the model with $\Omega / \Omega_{\text {crit }}=0.39$ becomes a RSG, and the core spins down rapidly. When it reaches core collapse its structure is extended, as implied by the large free-fall timescale 

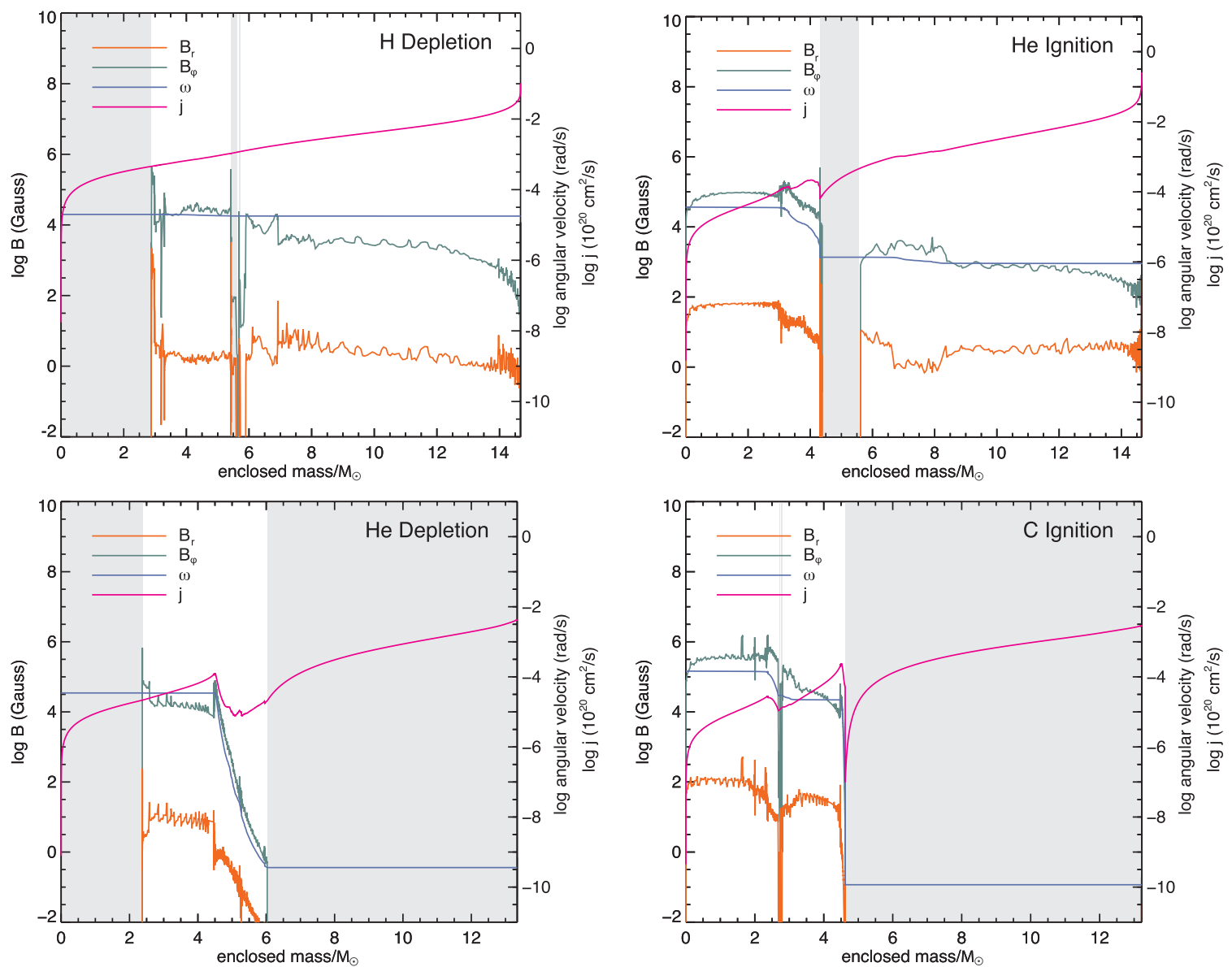

Figure 30. Magnetic field structure and angular momentum distribution for model 15MAG at different evolutionary stages (see Table 4). The curves show profiles for specific angular momentum $(j)$, angular velocity $(\omega)$, and azimuthal and radial components of magnetic field $\left(B_{\phi}\right.$ and $\left.B_{r}\right)$. The shaded regions represent convective parts of the star. Compare with Figure 1 in Heger et al. (2005).

(A color version of this figure is available in the online journal.)
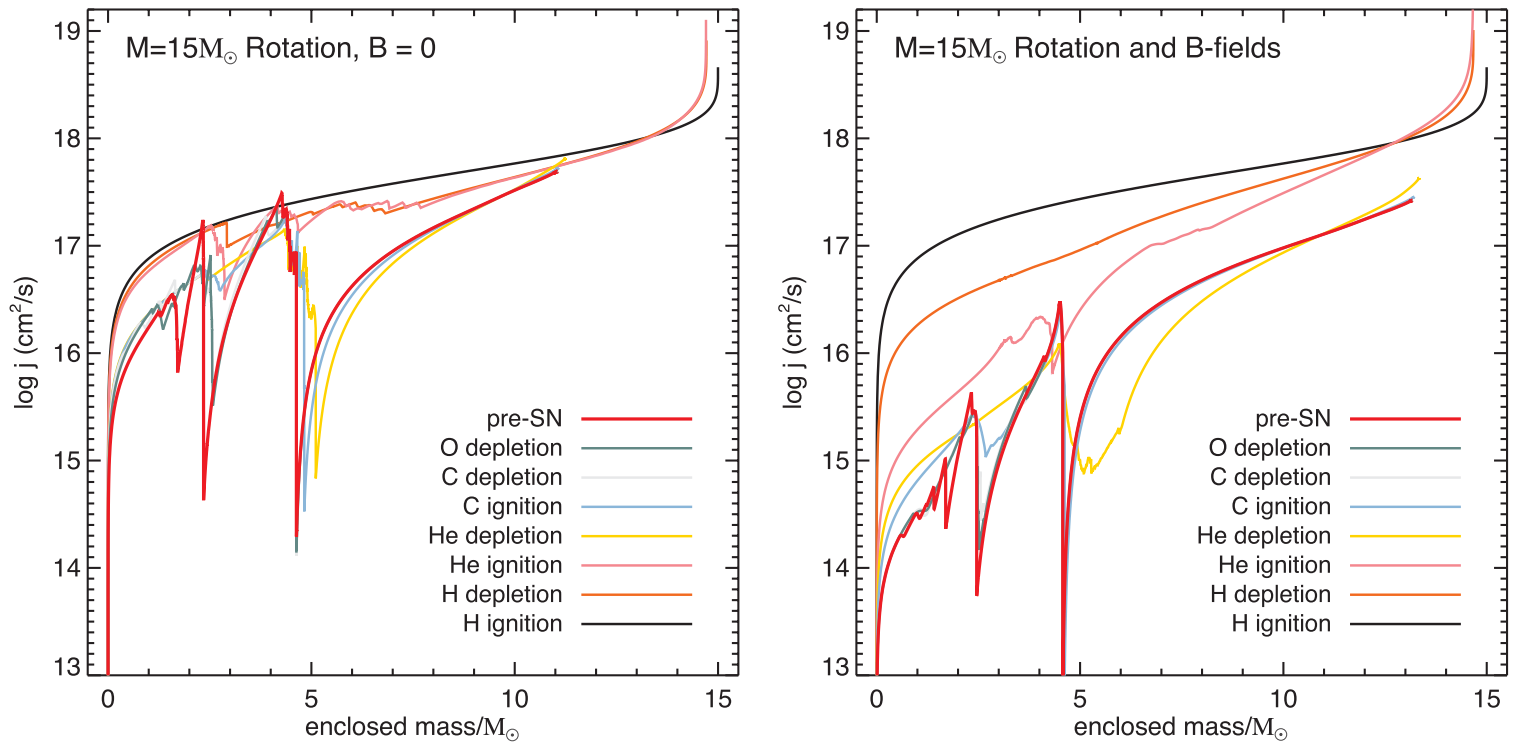

Figure 31. Specific angular momentum distribution at different evolutionary stage for 15MAG and 15ROT. See Table 4 for the definitions of these times. Compare with Figure 2 in Heger et al. (2005).

(A color version of this figure is available in the online journal.)

shown in the left panel of Figure 35. As a consequence, there is not enough angular momentum in its core to build an accretion disk around a newly formed compact object. This model is expected to produce a Type IIP supernova. On the contrary, the model with $\Omega / \Omega_{\text {crit }}=0.55$ is compact (the free-fall timescale is on the order of seconds, right panel of Figure 35 ) and has enough angular momentum to produce an accretion disk around the central compact object. Therefore 
Table 4

Evolution of Angular Momentum at Fiducial Mass Coordinates for a $Z=0.02,15 M_{\odot}$ Star Initially Rotating with $v_{\mathrm{eq}}=200 \mathrm{~km} \mathrm{~s}$ With (15MAG) and Without (15ROT) the Inclusion of Magnetic Fields

\begin{tabular}{|c|c|c|c|c|c|c|c|}
\hline \multirow[t]{2}{*}{ Evolution Stage } & & \multicolumn{3}{|c|}{$15 \mathrm{MAG}$} & \multicolumn{3}{|c|}{$15 \mathrm{ROT}$} \\
\hline & & $\mathrm{J}(1.5)$ & $\mathrm{J}(2.5)$ & $\mathrm{J}(3.5)$ & $\mathrm{J}(1.5)$ & $\mathrm{J}(2.5)$ & $\mathrm{J}(3.5)$ \\
\hline \multirow[t]{3}{*}{ ZAMS } & MESA & $1.82 \times 10^{50}$ & $4.38 \times 10^{50}$ & $7.90 \times 10^{50}$ & $1.82 \times 10^{50}$ & $4.38 \times 10^{50}$ & $7.90 \times 10^{50}$ \\
\hline & Kepler ${ }^{\mathrm{a}}$ & $1.75 \times 10^{50}$ & $4.20 \times 10^{50}$ & $7.62 \times 10^{50}$ & $2.30 \times 10^{50}$ & $5.53 \times 10^{50}$ & $1.00 \times 10^{51}$ \\
\hline & STERN $^{b}$ & $1.76 \times 10^{50}$ & $4.27 \times 10^{50}$ & $7.74 \times 10^{50}$ & $1.76 \times 10^{50}$ & $4.28 \times 10^{50}$ & $7.76 \times 10^{50}$ \\
\hline \multirow[t]{3}{*}{ H-burn ${ }^{c}$} & MESA & $1.25 \times 10^{50}$ & $3.03 \times 10^{50}$ & $5.51 \times 10^{50}$ & $1.64 \times 10^{50}$ & $3.99 \times 10^{50}$ & $7.26 \times 10^{50}$ \\
\hline & Kepler & $1.31 \times 10^{50}$ & $3.19 \times 10^{50}$ & $5.83 \times 10^{50}$ & $1.51 \times 10^{50}$ & $3.68 \times 10^{50}$ & $6.72 \times 10^{50}$ \\
\hline & STERN & $1.21 \times 10^{50}$ & $2.96 \times 10^{50}$ & $5.40 \times 10^{50}$ & $1.62 \times 10^{50}$ & $3.97 \times 10^{50}$ & $7.25 \times 10^{50}$ \\
\hline \multirow[t]{3}{*}{ H-dep ${ }^{d}$} & MESA & $4.32 \times 10^{49}$ & $1.08 \times 10^{50}$ & $2.03 \times 10^{50}$ & $1.54 \times 10^{50}$ & $3.86 \times 10^{50}$ & $6.44 \times 10^{50}$ \\
\hline & Kepler & $5.02 \times 10^{49}$ & $1.26 \times 10^{50}$ & $2.37 \times 10^{50}$ & $1.36 \times 10^{50}$ & $3.41 \times 10^{50}$ & $6.37 \times 10^{50}$ \\
\hline & STERN & $4.81 \times 10^{49}$ & $1.21 \times 10^{50}$ & $2.29 \times 10^{50}$ & $1.48 \times 10^{50}$ & $3.74 \times 10^{50}$ & $6.99 \times 10^{50}$ \\
\hline \multirow[t]{3}{*}{$\mathrm{He}-\mathrm{ign}^{\mathrm{e}}$} & MESA & $4.56 \times 10^{48}$ & $1.36 \times 10^{49}$ & $3.46 \times 10^{49}$ & $1.37 \times 10^{50}$ & $3.63 \times 10^{50}$ & $5.35 \times 10^{50}$ \\
\hline & Kepler & $4.25 \times 10^{48}$ & $1.21 \times 10^{49}$ & $2.57 \times 10^{49}$ & $1.16 \times 10^{50}$ & $2.98 \times 10^{50}$ & $4.87 \times 10^{50}$ \\
\hline & STERN & $4.10 \times 10^{48}$ & $1.16 \times 10^{49}$ & $3.25 \times 10^{49}$ & $1.33 \times 10^{50}$ & $3.47 \times 10^{50}$ & $6.36 \times 10^{50}$ \\
\hline \multirow[t]{2}{*}{ He-burn ${ }^{f}$} & MESA & $2.71 \times 10^{48}$ & $7.23 \times 10^{48}$ & $1.52 \times 10^{49}$ & $7.48 \times 10^{49}$ & $1.98 \times 10^{50}$ & $3.93 \times 10^{50}$ \\
\hline & STERN & $3.30 \times 10^{48}$ & $8.57 \times 10^{48}$ & $1.87 \times 10^{49}$ & $8.46 \times 10^{49}$ & $2.16 \times 10^{50}$ & $4.39 \times 10^{50}$ \\
\hline \multirow[t]{3}{*}{ He-dep ${ }^{g}$} & MESA & $2.10 \times 10^{48}$ & $5.65 \times 10^{48}$ & $1.22 \times 10^{49}$ & $5.40 \times 10^{49}$ & $1.44 \times 10^{50}$ & $2.81 \times 10^{50}$ \\
\hline & Kepler & $2.23 \times 10^{48}$ & $5.95 \times 10^{48}$ & $1.21 \times 10^{49}$ & $4.72 \times 10^{49}$ & $1.26 \times 10^{50}$ & $2.52 \times 10^{50}$ \\
\hline & STERN & $2.70 \times 10^{48}$ & $7.17 \times 10^{48}$ & $1.51 \times 10^{49}$ & $6.80 \times 10^{49}$ & $1.75 \times 10^{50}$ & $3.41 \times 10^{50}$ \\
\hline \multirow[t]{3}{*}{ C-ign ${ }^{h}$} & MESA & $1.54 \times 10^{48}$ & $5.21 \times 10^{48}$ & $8.89 \times 10^{48}$ & $5.40 \times 10^{49}$ & $1.44 \times 10^{50}$ & $2.58 \times 10^{50}$ \\
\hline & Kepler & $1.88 \times 10^{48}$ & $5.52 \times 10^{48}$ & $1.12 \times 10^{49}$ & $4.69 \times 10^{49}$ & $1.26 \times 10^{50}$ & $2.46 \times 10^{50}$ \\
\hline & STERN & $1.56 \times 10^{48}$ & $5.58 \times 10^{48}$ & $1.04 \times 10^{49}$ & $5.85 \times 10^{49}$ & $1.59 \times 10^{50}$ & $2.79 \times 10^{50}$ \\
\hline \multirow[t]{3}{*}{ C-dep ${ }^{\mathrm{i}}$} & MESA & $7.54 \times 10^{47}$ & $3.84 \times 10^{48}$ & $6.71 \times 10^{48}$ & $5.11 \times 10^{49}$ & $1.39 \times 10^{50}$ & $2.09 \times 10^{50}$ \\
\hline & Kepler & $8.00 \times 10^{47}$ & $3.26 \times 10^{48}$ & $9.08 \times 10^{48}$ & $4.06 \times 10^{49}$ & $1.25 \times 10^{50}$ & $2.24 \times 10^{50}$ \\
\hline & STERN & $9.04 \times 10^{47}$ & $4.48 \times 10^{48}$ & $9.33 \times 10^{48}$ & $5.04 \times 10^{49}$ & $1.56 \times 10^{50}$ & $2.61 \times 10^{50}$ \\
\hline \multirow[t]{2}{*}{ O-dep $\mathrm{p}^{\mathrm{j}}$} & MESA & $7.52 \times 10^{47}$ & $3.71 \times 10^{48}$ & $6.41 \times 10^{48}$ & $4.61 \times 10^{49}$ & $1.37 \times 10^{50}$ & $1.97 \times 10^{50}$ \\
\hline & Kepler & $7.85 \times 10^{47}$ & $3.19 \times 10^{48}$ & $8.43 \times 10^{48}$ & $3.94 \times 10^{49}$ & $1.20 \times 10^{50}$ & $1.99 \times 10^{50}$ \\
\hline \multirow[t]{2}{*}{ Si-dep ${ }^{k}$} & MESA & $7.28 \times 10^{47}$ & $3.64 \times 10^{48}$ & $5.90 \times 10^{48}$ & $4.03 \times 10^{49}$ & $1.22 \times 10^{50}$ & $1.76 \times 10^{50}$ \\
\hline & Kepler & $7.76 \times 10^{47}$ & $3.05 \times 10^{48}$ & $7.23 \times 10^{48}$ & $3.75 \times 10^{49}$ & $1.16 \times 10^{50}$ & $1.95 \times 10^{50}$ \\
\hline
\end{tabular}

Notes.

${ }^{a}$ Results from Table 1 of Heger et al. (2005).

b See, e.g., Petrovic et al. (2005); Yoon \& Langer (2005); Yoon et al. (2006).

c $40 \%$ central hydrogen mass fraction.

d $1 \%$ hydrogen left in the core.

e $1 \%$ helium burnt.

f $50 \%$ central helium mass fraction.

$\mathrm{g} 1 \%$ helium left in the core.

${ }^{\mathrm{h}}$ Central temperature of $5 \times 10^{8} \mathrm{~K}$.

${ }^{\mathrm{i}}$ Central temperature of $1.2 \times 10^{9} \mathrm{~K}$.

$\mathrm{j}$ Central oxygen mass fraction drops below $5 \%$.

${ }^{\mathrm{k}}$ Central Si mass fraction drops below $10^{-4}$.

this model is a candidate progenitor for a long gamma-ray burst (Woosley 1993). This last calculation can be directly compared to the Kepler model 16TI in Woosley \& Heger (2006).

We further test MESA capabilities by evolving two rotating $40 M_{\odot}$ models at $Z=10^{-5}$. One model is initialized at the ZAMS with $v_{\mathrm{eq}}=260 \mathrm{~km} \mathrm{~s}^{-1}$, while the other has $v_{\mathrm{eq}}=$ $630 \mathrm{~km} \mathrm{~s}^{-1}$. The results of these calculations can be compared with the models shown in Yoon \& Langer (2005). Figure 36 shows that for the more rapidly rotating model, rotational mixing (mainly due to the Eddington-Sweet circulation) is large enough that the star evolves blueward in the H-R diagram. This evolution results in a compact configuration and enough angular momentum to fulfill the requirements of the collapsar scenario for long gamma-ray bursts, as shown in Figure 37 (right panel). On the other hand, the slower rotating model becomes a RSG and loses most of its core angular momentum, as shown in Figure 37 (left panel).

\section{MASSIVE STELLAR EVOLUTION}

Modeling massive stars is numerically difficult. One problem is they develop loosely bound, radiation-pressure-dominated envelopes that can cause density and gas pressure inversions. Indeed, very massive stars are observed to suffer sporadic "eruptions" of extreme mass loss (i.e., the luminous blue variables), and the tendency to form inversions has been speculatively mentioned as playing a role in such episodes (see Humphreys \& Davidson 1994 and references therein). This environment poses a physical and numerical challenge that all stellar evolution codes must address to evolve massive stars past the MS. In this section we discuss MESAstar's capability to evolve rotating massive stars from their ZAMS to core collapse.

\subsection{Evolution of Massive Stars with MESA}

Previous computations with MESAstar found these envelopes to be numerically (and probably physically) unstable. This is 

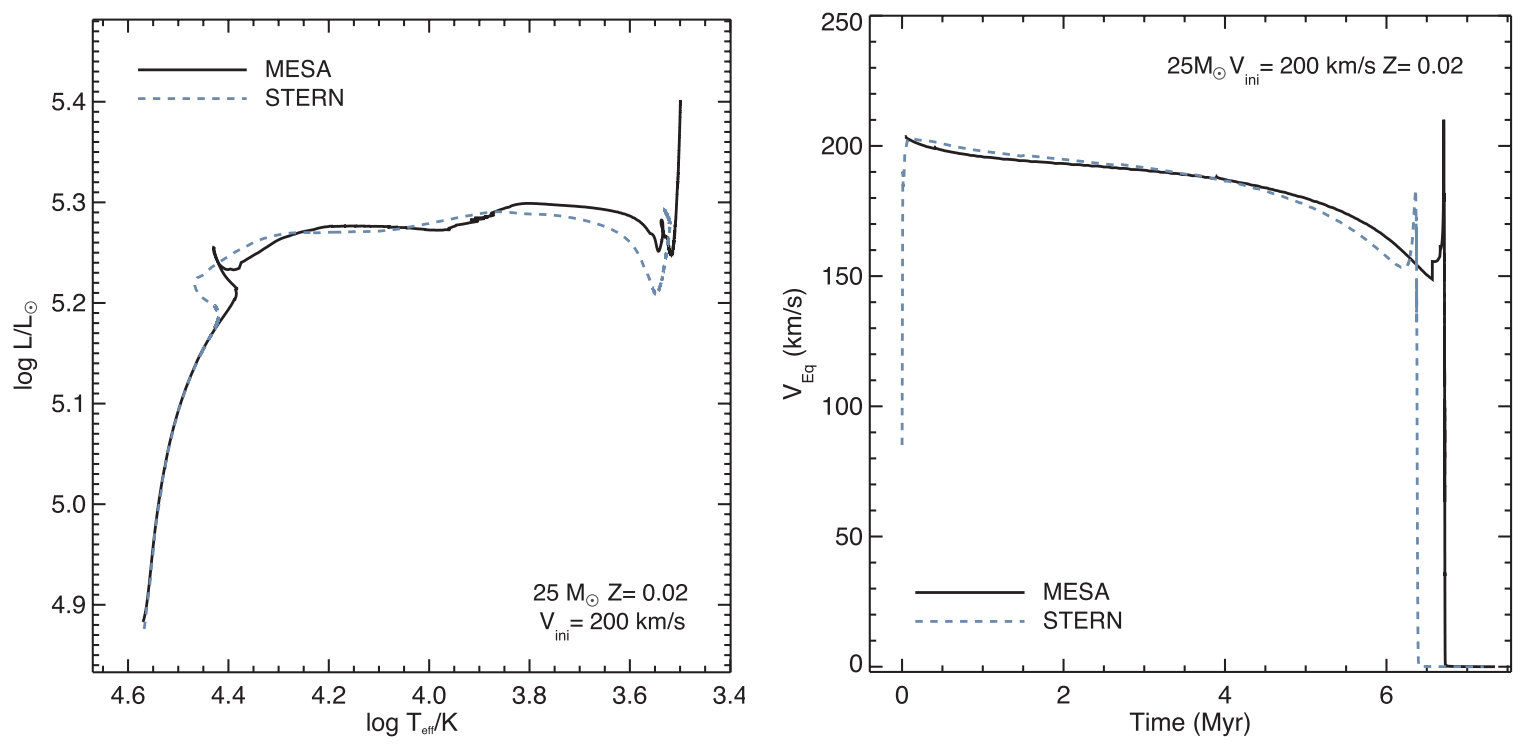

Figure 32. Same as Figure 28, except for the 25MAG model.

(A color version of this figure is available in the online journal.)
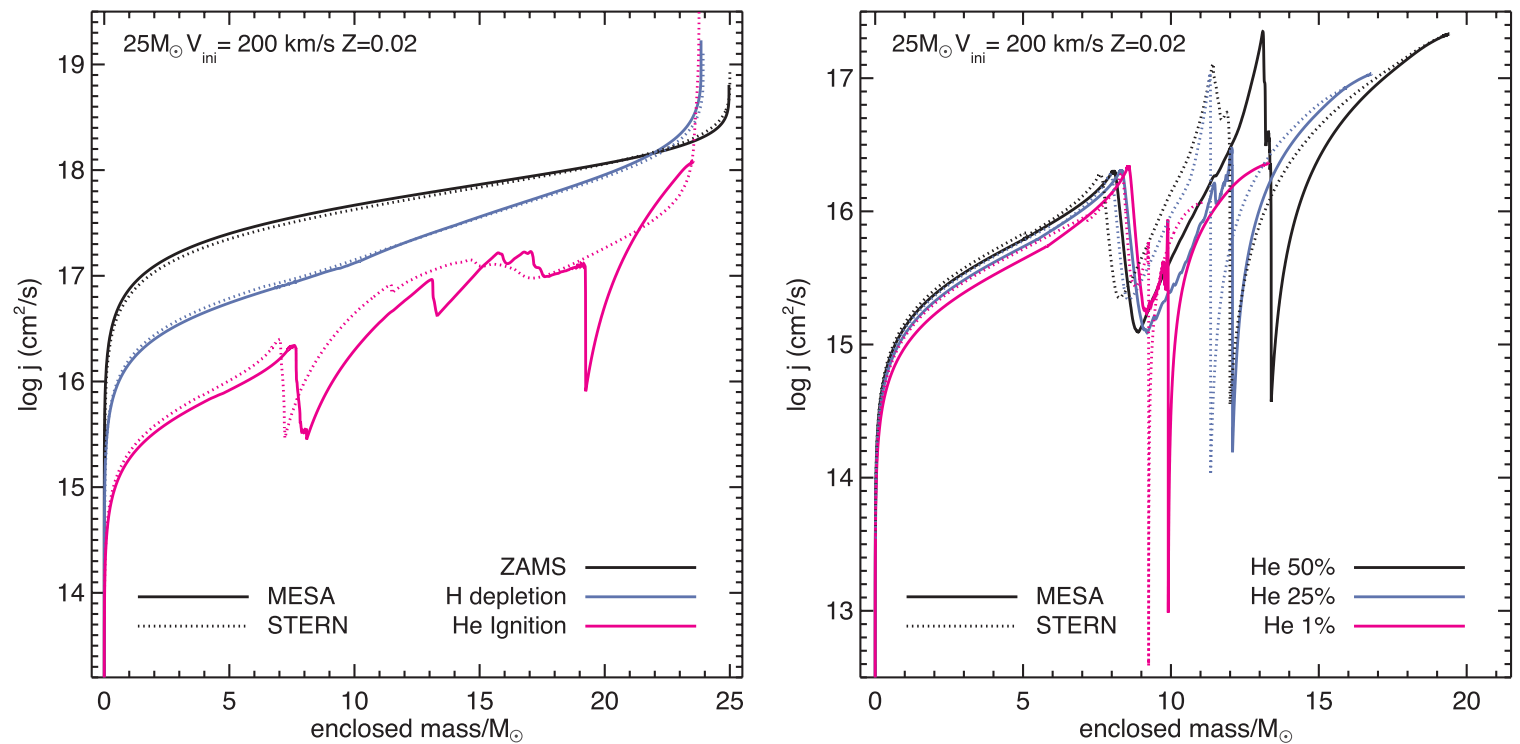

Figure 33. Evolution of internal specific angular momentum for the 25MAG model. Solid lines show MESAstar result, while dashed lines refer to STERN. Left panel shows the evolution from zero-age main sequence to He ignition. Right panel shows the evolution during core He-burning (from 50\% of He in the core to He depletion). Notice the different axis range in the two plots.

(A color version of this figure is available in the online journal.)

a known issue in the literature (e.g., Maeder 1987a), which reveals the limitations of the $1 \mathrm{D}$ treatment of late phases of evolution of massive stars. The evolution of stars with radiation-dominated envelopes can require prohibitively short time steps in MESAstar if the standard MLT is adopted. This problem usually appears during the evolution of high mass and/or high metallicity stars after hydrogen-core burning and prevents evolution to core collapse. We discuss in Section 7.2 our treatment of superadiabatic convection in these envelopes, which allows uninterrupted evolution, from ZAMS to core collapse.

Since it is relevant to later discussions we start with a plot of the OPAL opacity data (Iglesias \& Rogers 1996) and $60 M_{\odot}$ ZAMS models in Figure 38. The plot is inspired by Figure 1 of Cantiello et al. (2009). The left-hand panel of Figure 38 shows the OPAL data for five different $Z$ values at constant $X=0.7$ and $\log \left(\rho / T_{6}{ }^{3}\right)=-5$, where $T_{6}$ is the temperature in units of $10^{6} \mathrm{~K}$. The right-hand panel shows the opacity profiles of five $60 M_{\odot}$ ZAMS models for the same five $Z$ values. The model profiles exhibit the same general behavior in the opacity-temperature profile as the raw opacity data. Of particular importance are the iron opacity bumps that occur at $\log T \approx 5.3$ and 6.3. These bumps cause both the local radiation pressure to dominate and the luminosity to approach the Eddington luminosity $L_{\text {Edd }}$.

Where the pressure is dominated by radiation and $L_{\text {rad }}$ approaches $L_{\text {Edd }}$, specific conditions can be reached that cause convection and inversions in density and gas pressure. To define the conditions under which these occur, we follow the discussion of Joss et al. (1973), going from high to low $L_{\text {rad. }}$ We assume that $d T / d r<0, d P / d r<0$, and that the inertial terms in the 


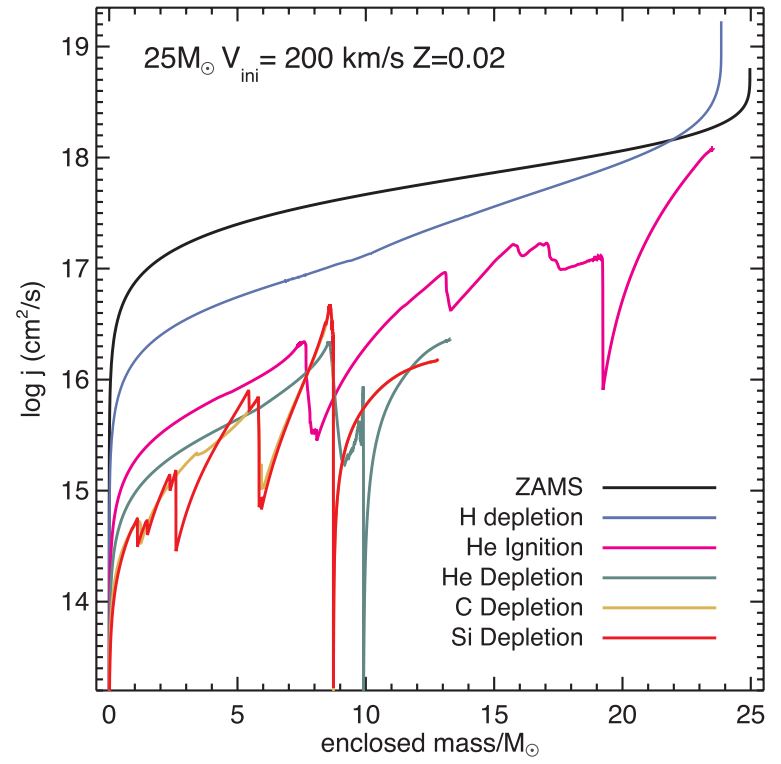

Figure 34. Evolution to Si depletion of the internal specific angular momentum for the 25MAG model.

(A color version of this figure is available in the online journal.)

momentum equation are small. First, we establish a condition for the occurrence of an inversion in the gas pressure $P_{\text {gas }}$. Recasting the equation for the temperature gradient gives

$$
L_{\mathrm{rad}}=-\frac{4 \pi r^{2} c}{\rho \kappa} \frac{d P_{\mathrm{rad}}}{d r},
$$

and using the equation of hydrostatic equilibrium, one obtains

$$
\frac{d P_{\mathrm{rad}}}{d P}=\frac{L_{\mathrm{rad}}}{L_{\mathrm{Edd}}} .
$$

Writing $d P_{\mathrm{gas}} / d r=d\left(P-P_{\mathrm{rad}}\right) / d r$ and using Equation (29) and the fact that both $P_{\mathrm{rad}}$ and $P$ monotonically decrease with $r$,

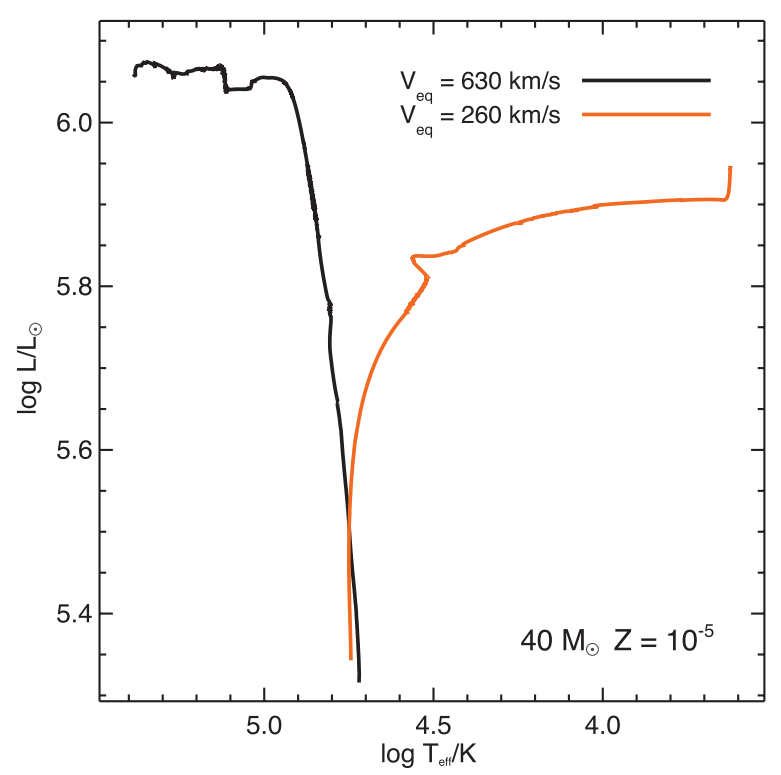

Figure 36. Evolution in the H-R diagram for two rotating $40 M_{\odot}$ models at $Z=10^{-5}$. The slower rotating model evolves toward the red part of the H-R diagram; the other model evolves toward the blue part of the H-R diagram. The internal evolution of the angular momentum is shown in Figure 37. This can be compared to Figure 2 of Yoon \& Langer (2005).

(A color version of this figure is available in the online journal.)

one obtains

$$
\frac{d P_{\mathrm{gas}}}{d r}=\left(\frac{d P_{\mathrm{rad}}}{d r}\right)\left[\frac{L_{\mathrm{Edd}}}{L_{\mathrm{rad}}}-1\right]
$$

Since $d P_{\mathrm{rad}} / d r<0$, Equation (30) implies that for $L_{\mathrm{rad}}>L_{\mathrm{Edd}}$, the gas pressure gradient will increase outward, $d P_{\text {gas }} / d r>0$, as shown by Joss et al. (1973).

The next step is to establish the condition for a density inversion to occur. Writing the gas EOS as $P_{\text {gas }}=P_{\text {gas }}\left(\rho, P_{\text {rad }}\right)$
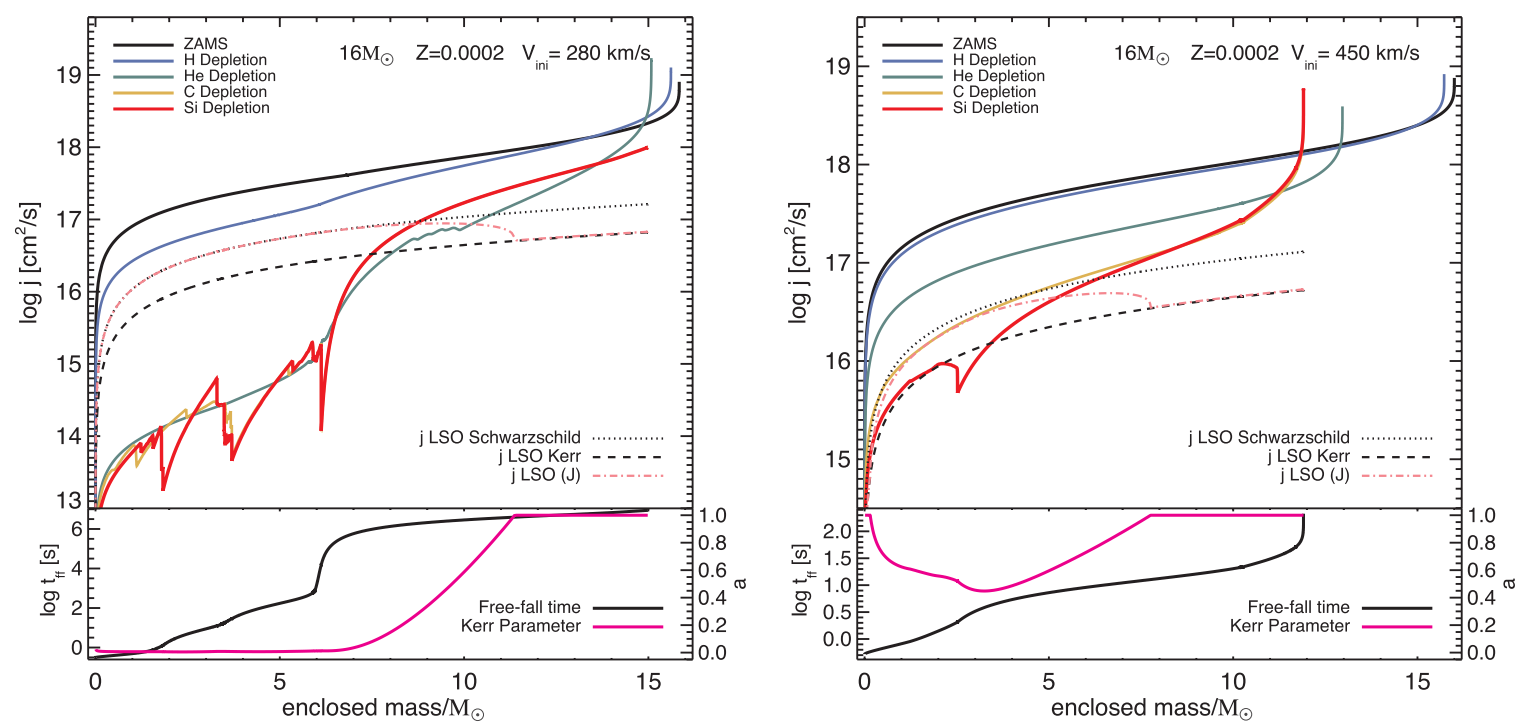

Figure 35. Specific angular momentum distribution for the two $16 M_{\odot}$ models. In the top panels, the solid curves show the distribution of specific angular momentum at different evolutionary stages. The other curves in the top panel show the specific angular momentum of the last stable orbit around a Schwarzschild black hole, a maximally rotating Kerr black hole $(a=1)$, and a black hole with a Kerr parameter corresponding to the angular momentum content of the stellar progenitor at that mass coordinate. The bottom panels show the free-fall time at the relative mass coordinate at the end of Si-burning. Notice the different ranges of the $y$-axis. These models can be compared to the calculations of Woosley \& Heger (2006), in particular their models 16SG and 16TI, respectively.

(A color version of this figure is available in the online journal.) 

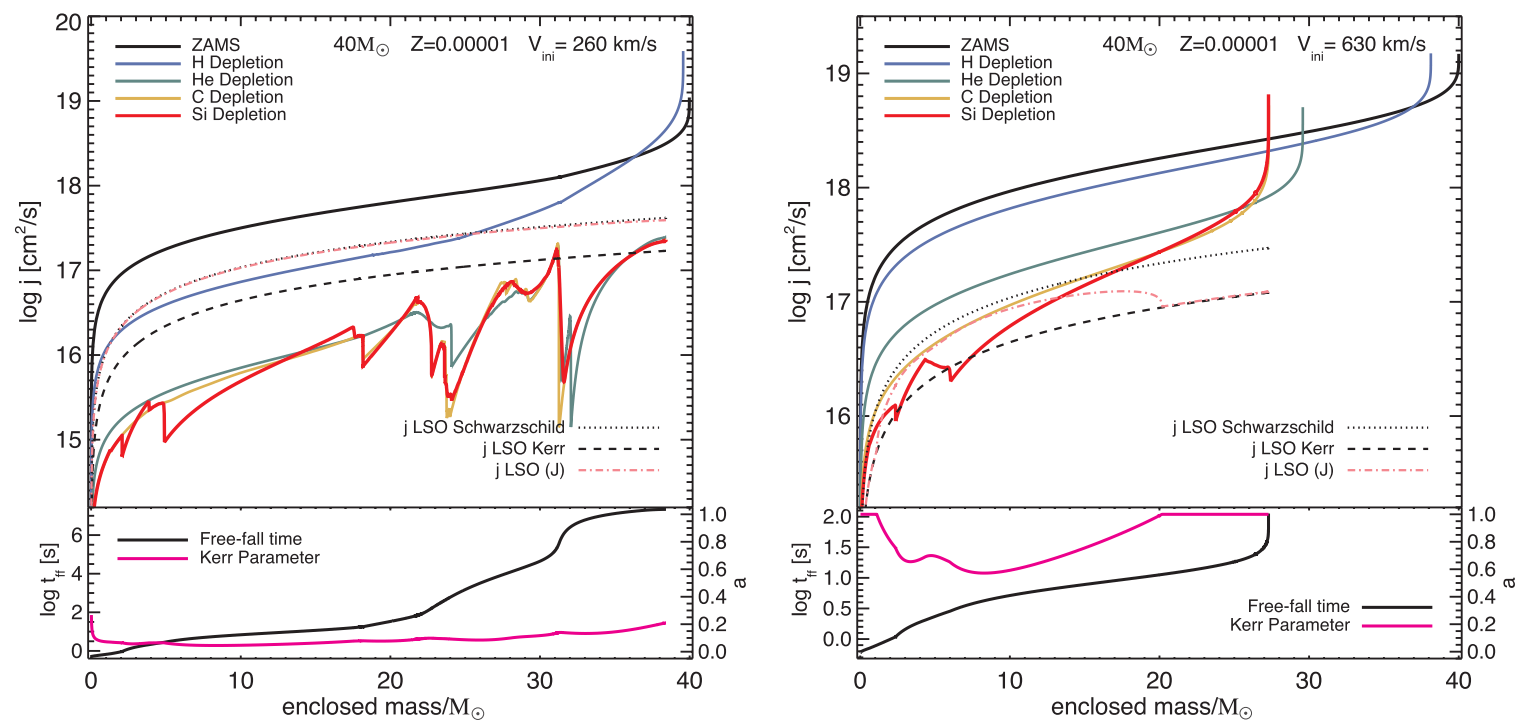

Figure 37. Specific angular momentum distribution for two $40 M_{\odot}$ models at $Z=10^{-5}$ with $v_{\mathrm{eq}}=260 \mathrm{~km} \mathrm{~s}^{-1}$ (left panel) and $630 \mathrm{~km} \mathrm{~s}{ }^{-1}$ (right panel). Lines are showing the distribution of specific angular momentum at different stages of the evolution, together with the specific angular momentum of the last stable orbit around a Schwarzschild black hole, a maximally rotating Kerr black hole $(a=1)$, and a black hole with a Kerr parameter corresponding to the angular momentum content of the stellar progenitor at that mass coordinate. Note the different ranges of the $y$-axis. The evolutionary tracks for these models are shown in Figure 36 . These calculations should be compared to Figure 5 of Yoon \& Langer (2005).

(A color version of this figure is available in the online journal.)
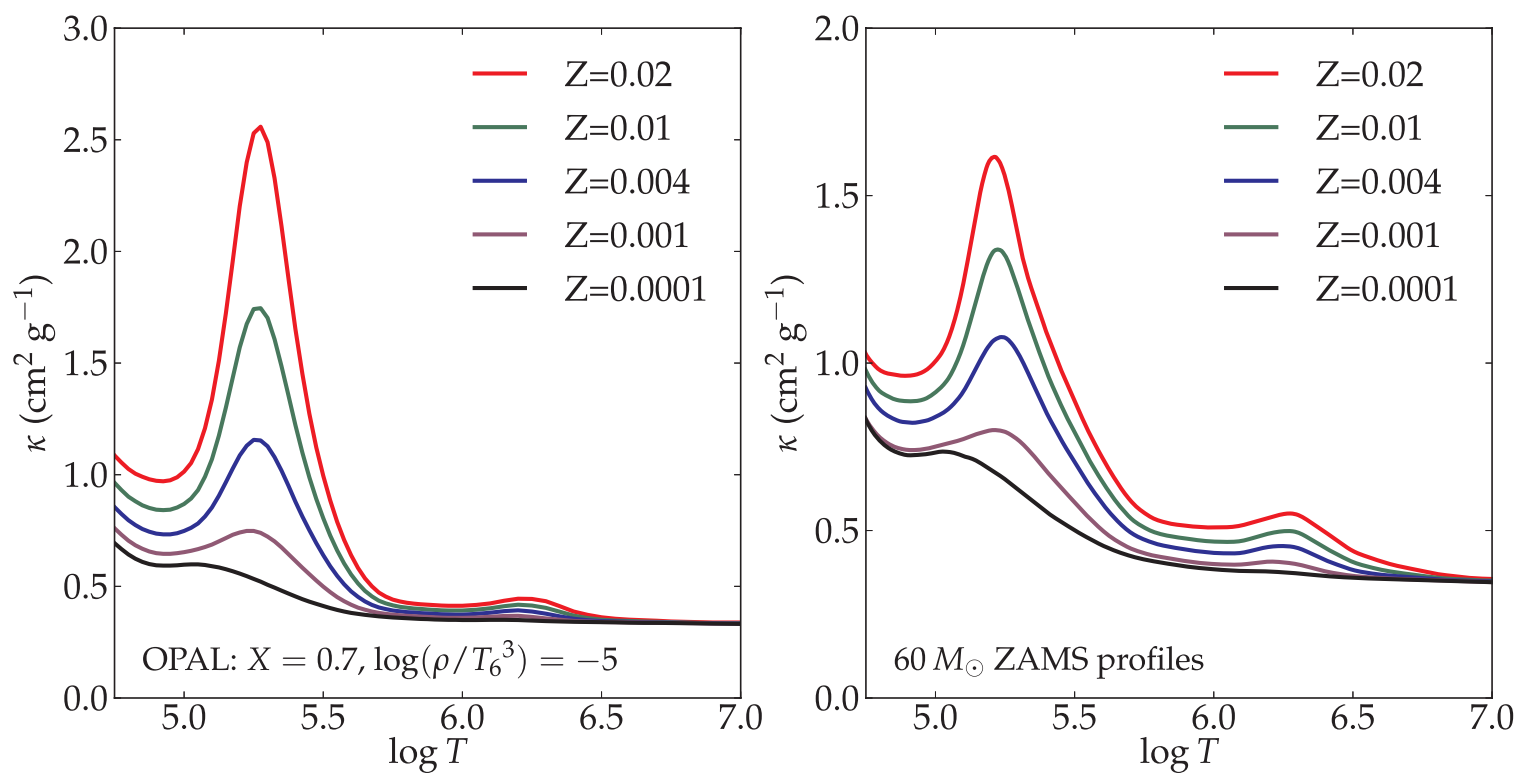

Figure 38. Left: a plot of the OPAL opacity data for five $Z$ values at $X=0.7$ and $\log \left(\rho / T_{6}{ }^{3}\right)=-5$. These curves show the increase in the iron opacity bumps at $\log T \approx 5.3$ and 6.3 as $Z$ increases from $10^{-4}$ to 0.02 . Right: the opacity-temperature profiles of $60 M_{\odot}$ ZAMS models for the same $Z$ values.

(A color version of this figure is available in the online journal.)

gives

$$
\frac{d P_{\mathrm{gas}}}{d r}=\left(\frac{\partial P_{\mathrm{gas}}}{\partial \rho}\right)_{P_{\mathrm{rad}}} \frac{d \rho}{d r}+\left(\frac{\partial P_{\mathrm{gas}}}{\partial P_{\mathrm{rad}}}\right)_{\rho} \frac{d P_{\mathrm{rad}}}{d r}
$$

Solving Equation (31) for $d \rho / d r$ and using Equation (30) eliminates $d P_{\text {gas }} / d r$. Gas equations of state have $\left(\partial P_{\text {gas }} / \partial \rho\right)_{T}>$ 0 , so that for $d \rho / d r>0$ (a density inversion), one must have

$$
\left(\frac{d P_{\mathrm{rad}}}{d r}\right)\left[\frac{L_{\mathrm{Edd}}}{L_{\mathrm{rad}}}-1-\left(\frac{\partial P_{\mathrm{gas}}}{\partial P_{\mathrm{rad}}}\right)_{\rho}\right]>0 .
$$

Recognizing that $d P_{\text {rad }} / d r<0$, we find that a density inversion occurs when

$$
\frac{L_{\mathrm{rad}}}{L_{\mathrm{Edd}}}>\frac{L_{\text {inv }}}{L_{\mathrm{Edd}}} \equiv\left[1+\left(\frac{\partial P_{\mathrm{gas}}}{\partial P_{\mathrm{rad}}}\right)_{\rho}\right]^{-1} .
$$

This equation is identical to Equation (8) of Joss et al. (1973). Since under conditions of interest $\left(\partial P_{\text {gas }} / \partial P_{\text {rad }}\right)_{\rho}>0$ we have $L_{\text {inv }}<L_{\text {Edd }}$. For $L_{\text {inv }}<L_{\text {rad }}<L_{\text {Edd }}$, a density inversion will occur even though $d P_{\text {gas }} / d r<0$.

Next, we shall consider the luminosity $L_{\text {onset }}$ at which convection occurs. In a convective region, the entropy is either constant or declining with radius. Hence, convection will occur 


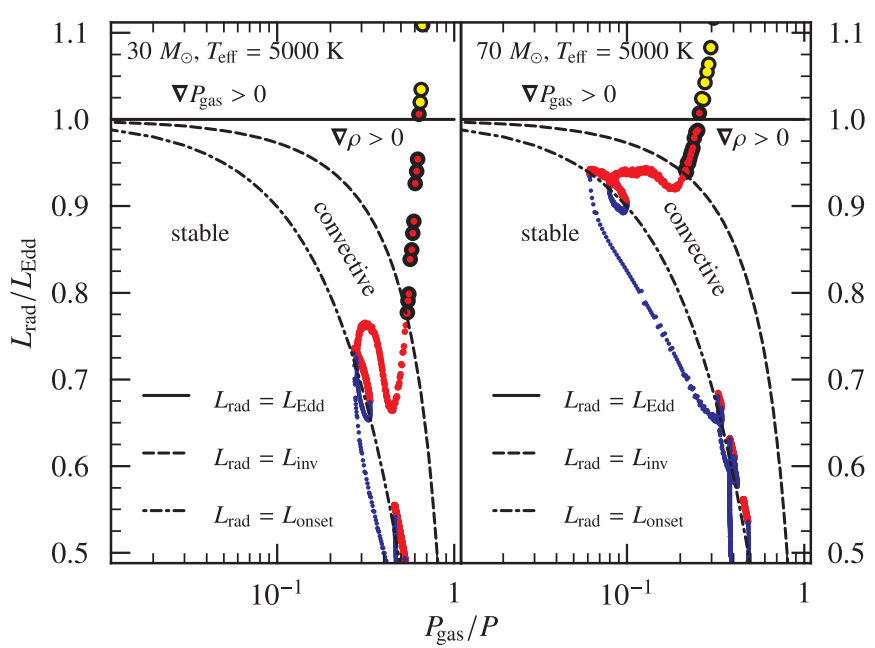

Figure 39. Critical luminosities $L_{\mathrm{rad}}=L_{\text {onset }}$ (Equation (36), dot-dashed line), $L_{\mathrm{rad}}=L_{\mathrm{inv}}$ (Equation (35), dashed line), and $L_{\mathrm{rad}}=L_{\mathrm{Edd}}$ (solid line) as a function of $P_{\text {gas }} / P$ for an ideal gas-radiation mixture. Compare this with Figure 1 of Joss et al. (1973). For $L_{\text {rad }}<L_{\text {onset }}$, the gas is convectively stable; for $L_{\text {onset }}<L_{\text {rad }}<L_{\text {inv }}$, the gas is convective; for $L_{\text {inv }}<L_{\text {rad }}<L_{\text {Edd }}$, the density is inverted, $d \rho / d r>0$; and for $L_{\mathrm{Edd}}<L_{\mathrm{rad}}$, the gas pressure is inverted, $d P_{\text {gas }} / d r>0$. Overlaid on the plots are the profiles from a $30 M_{\odot}$ (left panel) and a $70 M_{\odot}$ (right panel) model with $Z=0.02$ : blue dots indicate zones that are radiative; red dots indicate $\nabla_{\mathrm{rad}}>\nabla_{\mathrm{ad}}$; dots with a black border have a density inversion; and the yellow dots with black borders indicate a gas pressure inversion. As the profile moves out from the stellar center it traces out the points on the plot from bottom to top. Only part of the model profiles is visible in the plot. The calculations correspond to the first crossing of the Hertzsprung gap when $T_{\text {eff }}=5000 \mathrm{~K}$

(A color version of this figure is available in the online journal.)

once

$$
\frac{d \ln P_{\mathrm{rad}}}{d \ln P}>\left(\frac{\partial \ln P_{\mathrm{rad}}}{\partial \ln P}\right)_{s} ;
$$

using Equation (29) and solving for the luminosity, we find that convection starts once

$$
\frac{L_{\mathrm{rad}}}{L_{\mathrm{Edd}}}>\frac{L_{\mathrm{onset}}}{L_{\mathrm{Edd}}} \equiv\left(1-\frac{P_{\mathrm{gas}}}{P}\right)\left(\frac{\partial \ln P_{\mathrm{rad}}}{\partial \ln P}\right)_{s} .
$$

Equation (34) corresponds to Equation (9) of Joss et al. (1973). As argued in that paper, entropy decreases as density increases; therefore a density inversion implies a superadiabatic gradient, and as a result, $L_{\text {onset }}<L_{\text {inv }}$. This can be shown explicitly for a chemically homogenous mixture of an ideal gas and radiation. For such a mixture, Equation (32) becomes

$$
\frac{L_{\mathrm{rad}}}{L_{\mathrm{Edd}}}>\frac{L_{\mathrm{inv}}}{L_{\mathrm{Edd}}}=\left[\frac{1-P_{\mathrm{gas}} / P}{1-3 P_{\mathrm{gas}} / 4 P}\right]
$$

and Equation (34) becomes

$$
\frac{L_{\mathrm{rad}}}{L_{\mathrm{Edd}}}>\frac{L_{\mathrm{onset}}}{L_{\mathrm{Edd}}}=\frac{8\left(1-P_{\mathrm{gas}} / P\right)\left(4-3 P_{\mathrm{gas}} / P\right)}{32-24 P_{\mathrm{gas}} / P+3\left(P_{\mathrm{gas}} / P\right)^{2}},
$$

allowing one to show that $L_{\text {onset }}<L_{\text {inv }}$. At high luminosities where the gas becomes radiation-dominated, however, the difference between $L_{\text {onset }}$ and $L_{\text {inv }}$ becomes small. Expanding Equations (35) and (36) for $P_{\text {gas }} / P \ll 1$ gives $L_{\text {inv }}-L_{\text {onset }} \approx$ $(3 / 4) \times\left(P_{\text {gas }} / P\right) \times L_{\text {Edd }}$. For such high-luminosity, radiationdominated stars, a small inefficiency in convection is sufficient to drive a density inversion.
We now demonstrate that such inefficient convection can arise in the convective, radiation-dominated, envelopes of massive stars. In order of magnitude the convective and radiative fluxes are, respectively, $F_{\text {conv }} \sim \rho c_{s}^{3}\left(\nabla_{T}-\nabla_{\mathrm{ad}}\right)^{3 / 2}$ and $F_{\text {rad }} \sim c P_{\text {rad }} / \tau$. To carry the flux, we need $F_{\text {conv }} \sim F_{\text {rad }}$; equating and substituting $\rho c_{s}^{2} \sim P \sim P_{\text {gas }}$, we arrive at an expression that sets the level of superadiabaticity,

$$
\left(\nabla_{T}-\nabla_{\mathrm{ad}}\right)^{3 / 2} \sim \frac{c}{c_{\mathrm{s}}} \frac{P_{\mathrm{rad}}}{P_{\mathrm{gas}}} \tau^{-1}
$$

Under typical conditions in massive star envelopes, $c / c_{\mathrm{s}} \sim 10^{4}$ at the iron opacity bump, but at this location, $\tau$ is not large enough to prevent the superadiabaticity from triggering a density inversion.

The lines in Figure 39 show these luminosity conditions as a function of $P_{\text {gas }} / P$, and reveal that as the stellar conditions become radiation dominated, there is only a small gap between a convective model that is adiabatically stratified and a model with a density inversion. This corresponds to the region between the curves $L_{\text {rad }}=L_{\text {onset }}$ (dot-dashed line) and $L_{\text {rad }}=L_{\text {inv }}$ (dashed line). The gas pressure does not invert until $L>L_{\text {Edd }}$, which in Figure 39 is the region above the solid horizontal line. We show profiles from a $30 M_{\odot}$ (left panel) and a $70 M_{\odot}$ model (right panel). These are from the first crossing of the Hertzsprung gap when $T_{\text {eff }}=5000 \mathrm{~K}$. Each dot corresponds to a zone in the calculation; as the profile moves outward from center to surface the traces go from bottom to top in the plot. The blue dots indicate zones where the star is radiative; red indicates convection; a black border denotes a density inversion, $d \rho / d r>0$; and yellow indicates a gas pressure inversion, $d P_{\text {gas }} / d r>0$. There is excellent agreement between the detailed MESA evolutionary calculations and the analytical conditions (Equation (35) and (36)). The $70 M_{\odot}$ profile goes into the low $P_{\text {gas }} / P$, high $L_{\text {rad }} / L_{\text {Edd }}$ regime.

Figure 40 displays the physical conditions in the $70 M_{\odot}$ model where the density and gas pressure inversions develop. The panels display, from top to bottom, density, gas pressure, total pressure, and entropy, all as functions of radius. The total radius is $R=1330 R_{\odot}$. Regions with $\nabla>\nabla_{\mathrm{ad}}$ and $L_{\text {rad }}<L_{\text {inv }}<L_{\text {Edd }}$ are marked with a small red dot. Regions where $L_{\text {inv }}<L_{\text {rad }}<L_{\text {Edd }}$ (cf. Equation (35)) are marked with a large red dot with a black border. Regions where $L_{\text {rad }}>L_{\text {Edd }}$ are marked with a large yellow dot with black border. Although the pressure (panel (c)) is well-behaved in this superadiabatic (panel (d)) region, a density inversion does develop where $L_{\text {Edd }}>L_{\text {rad }}>L_{\rho}$ (panel (a)) and a gas pressure inversion develops (panel (b)) where $L_{\text {rad }}>L_{\text {Edd }}$, as predicted. In this region the superadiabaticity $\nabla_{T}-\nabla_{\mathrm{ad}}>10^{-2}$ and is greater than unity for $r / R_{\odot} \gtrsim 1300$. This is much larger than a typical value $\left(\sim 10^{-6}\right)$ where convection is efficient and results in the entropy decreasing with $r$ as shown in panel (c).

\subsection{Treatment of Superadiabatic Convection in Radiation-dominated Regions}

In MESAstar the superadiabatic gradient arising in radiationdominated envelopes can force the adoption of prohibitively short time steps. Energy is mostly transported by radiation, and the convective velocities resulting from MLT approach the sound speed. The stability of such radiation-dominated envelopes has been discussed in the past, and is still a matter of debate (see, e.g., Langer 1997; Bisnovatyi-Kogan \& Dorodnitsyn 1999; Maeder 2009; Suárez-Madrigal et al. 2013). In this 


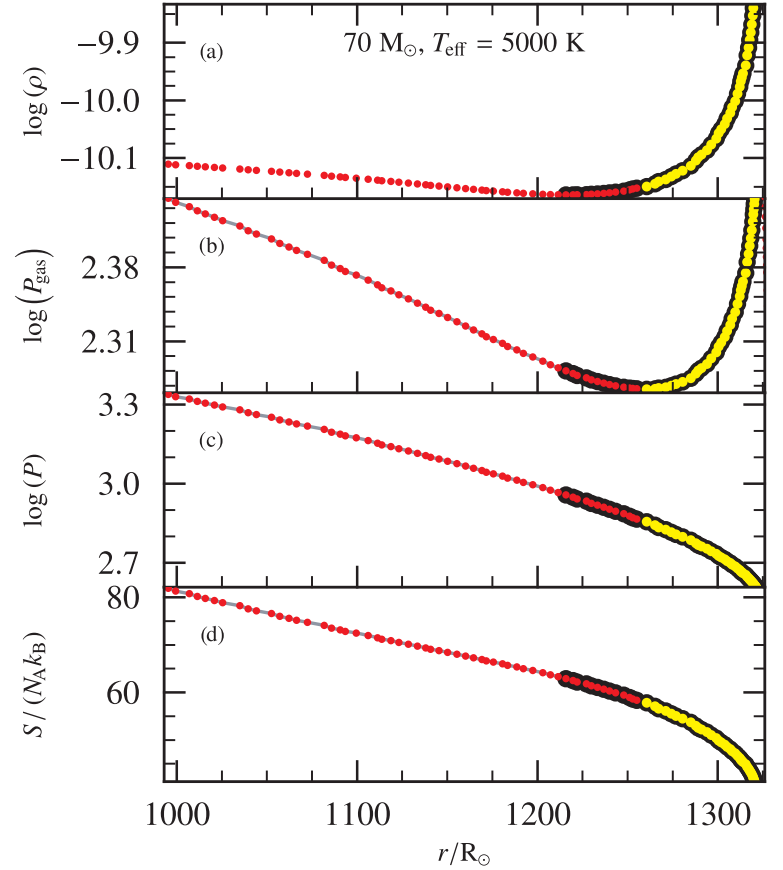

Figure 40. Panels display, from top to bottom, the density, gas pressure, total pressure, and entropy as functions of radius for the $70 M_{\odot}$ model shown in Figure 39. The range of radii is restricted to the region where density and gas pressure inversions develop. Each zone is marked by a dot; a small red dot indicates convection with no predicted gas or gas pressure inversion ( $\left.L_{\text {rad }}<L_{\text {inv }}\right)$; a large red dot with black border indicates a predicted density inversion but no gas pressure inversion ( $L_{\text {inv }}<L_{\text {rad }}<L_{\text {Edd }}$ ); and a yellow dot with black border indicates a convective region with a predicted gas pressure inversion ( $L_{\mathrm{rad}}>L_{\text {Edd }}$ ). The total pressure (panel (c)) is well-behaved at all radii. Note also the decrease in entropy (panel (d)): the region is superadiabatic.

(A color version of this figure is available in the online journal.)

regime, the treatment of convective energy transport by MLT is admittedly out of its domain of applicability. Hydrodynamical instabilities and the transport of energy from waves excited by near-sonic turbulent convection are important for energy transport, and 3D hydrodynamical calculations are required to capture fully the complex physics occurring in this regime.

Here we develop a treatment of convection, known as MLT++, that reduces the superadiabaticity in some radiation-dominated convective regions. This treatment allows MESAstar to calculate models of massive stars up to core collapse. For every model, MESAstar computes the values of

$$
\lambda_{\max } \equiv \max \left(\frac{L_{\mathrm{rad}}}{L_{\mathrm{Edd}}}\right) \quad \text { and } \quad \beta_{\min } \equiv \min \left(\frac{P_{\mathrm{gas}}}{P}\right) .
$$

When $\beta_{\min }$ is small and $\lambda_{\max }$ is large, and MLT yields a $\delta_{\nabla}>\delta_{\nabla \text {,thresh }}$, we artificially decrease the superadiabaticity, $\delta_{\nabla} \equiv \nabla_{T}-\nabla_{\text {ad }}$, implied by MLT. The default of the user-

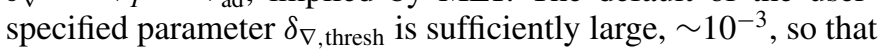
convection is still inefficient.

MESAstar sets $\nabla_{T}$ to reduce the $\delta_{\nabla}-\delta_{\nabla \text {,thresh by a factor }}$ $\alpha_{\nabla} f_{\nabla}$, where $f_{\nabla}$ is specified by the user, and $\alpha_{\nabla}$ is updated at each time step to a linear combination of its previous value and a value $\widetilde{\alpha_{\nabla}}\left(\lambda_{\max }, \beta_{\min }\right)$. For large values of $\lambda_{\max }$ and small values of $\beta_{\min }, \widetilde{\alpha_{\nabla}} \rightarrow 1$; in typical usage, the transition happens where $\lambda_{\max } \approx 0.5$ and $\beta_{\min } \approx 0.3$. For small values of $\lambda_{\max }$ and large values of $\beta_{\min }, \widetilde{\alpha_{\nabla}} \rightarrow 0$. Thus $f_{\nabla}$ sets the maximum reduction

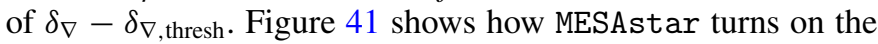
reduction in $\delta_{\nabla}$ as a star evolves. Tracks in the H-R diagram are

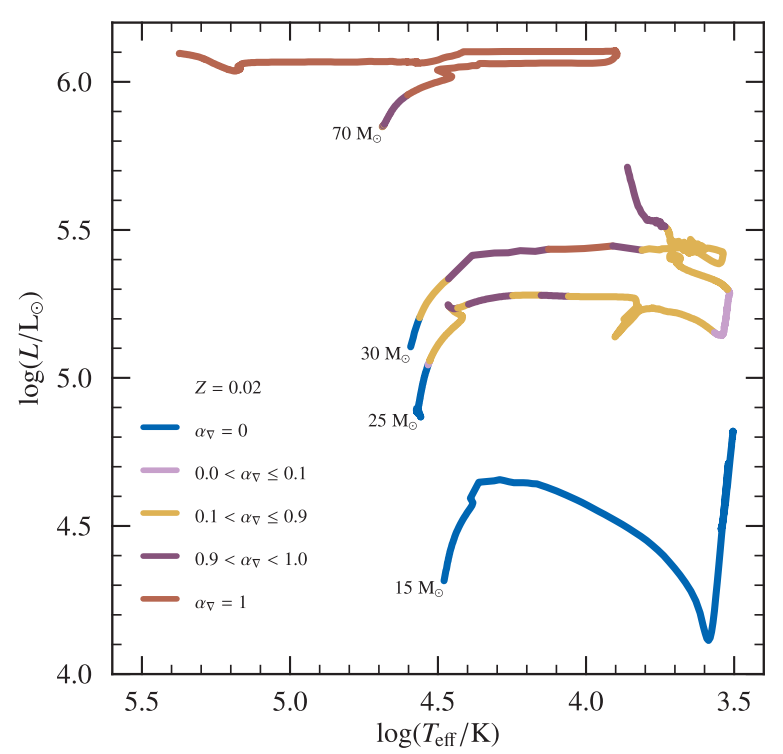

Figure 41. $\mathrm{H}-\mathrm{R}$ diagram of $15,25,30$, and $70 M_{\odot}$ models. The color indicates the value of $\alpha_{\nabla}$ at that point in the star's evolution. For the $25 M_{\odot}$ and $30 M_{\odot}$ stars, there is a sharp spike in $\alpha_{\nabla}$ as the star crosses the Hertzsprung gap followed by a sharp drop at the base of the red giant branch. The $70 M_{\odot}$ model has $\alpha_{\nabla}>0.9$ for its entire evolution.

(A color version of this figure is available in the online journal.)

shown for four stellar models: $15,25,30$, and $70 M_{\odot}$. The color of each line indicates the value of $\alpha_{\nabla}$ at each point.

Such a decrease of the temperature gradient reduces $L_{\text {rad }}$ and implies additional physical transport. Potential agents for the excess transport include waves excited by turbulent convection (see, e.g., Maeder 1987a) and radiative diffusion enhanced by porous clumping of the envelope (e.g., Owocki et al. 2004). As these radiation-dominated envelopes might be physically unstable, with a resulting strong enhancement of mass loss, we caution that the results of any $1 \mathrm{D}$ stellar evolution calculation for the late evolutionary phases of massive stars should be considered highly uncertain.

We now show a comparison of MESAstar calculations of rotating massive stars done with and without MLT++. We used the $25 M_{\odot}$ model described in Section 6.6, which at $Z=0.02$ is around the upper mass limit that can converge using a reasonably short time step without having to rely on the MLT++. The most prominent difference between the calculations is the evolutionary track in the H-R diagram (Figure 42). This is not surprising, as MLT and MLT++ result in different efficiencies of energy transport in radiation-dominated stellar envelopes. The sharp drop in $L$ for the MLT++ case is the result of a brief period of enhanced mass loss due to super-critical rotation. The structure and the angular momentum content of the collapsing core are weakly dependent, however, on the choice of MLT versus MLT++ (Figure 43).

\subsection{Core-collapse Progenitor Models}

We evolve a grid of massive stars initially rotating with $\Omega / \Omega_{\text {crit }}=0.2$. The models have been initialized using solid body rotation. Models with initial $M / M_{\odot}=30,40,50,60$, 70, 80, 90, and 100 have initial $Z=0.02$, while models with initial $M / M_{\odot}=120,150,250,500$ and 1000 have been initialized with $Z=0.001$. To calculate convective boundaries we adopt the Ledoux criterion including the impact of semiconvection (with $\alpha_{\mathrm{sc}}=0.02$; see Section 4.1). The 

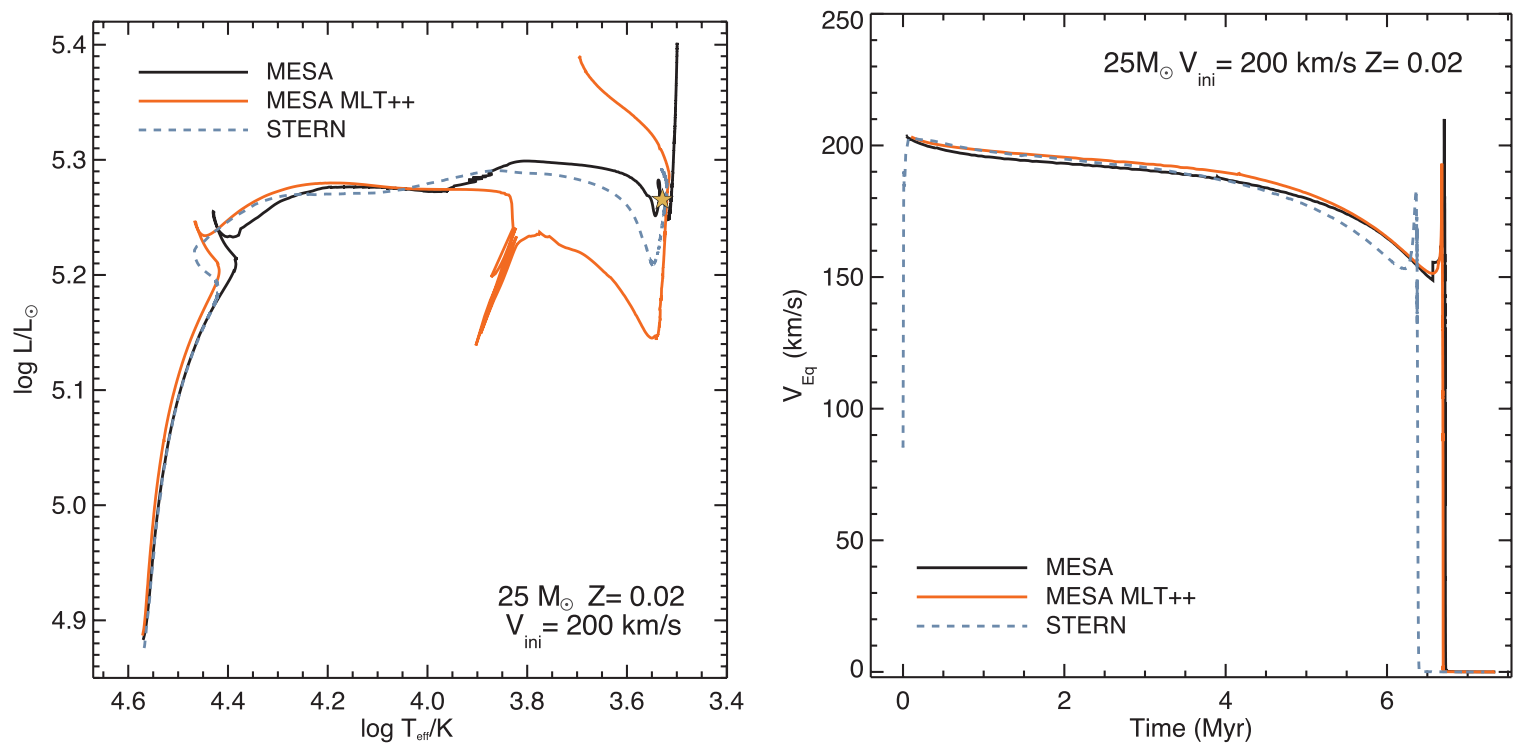

Figure 42. Comparison of evolutionary tracks (left) and equatorial rotational velocity (right) for a $25 M_{\odot}$ model with $Z=0.02$ and $v_{\mathrm{eq}}=200 \mathrm{~km} \mathrm{~s}^{-1}$. The solid black lines show MESAstar results with MLT (black) and MLT++ (orange), while the dashed blue line refers to STERN calculations. The star symbol shows the location where we started the calculation for the RSG pulsations discussed in Section 7.4.

(A color version of this figure is available in the online journal.)

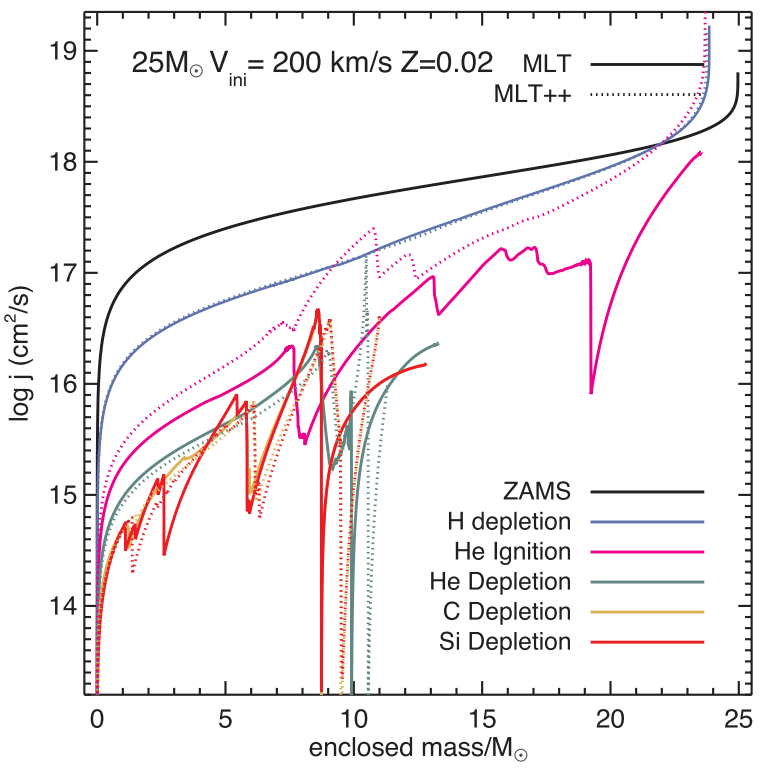

Figure 43. Evolution of internal specific angular momentum for the two $25 M_{\odot}$ models of Figure 42. The dashed lines show models calculated with MLT++. Due to different excursions in the H-R diagram (see Figure 28) calculations with MLT and MLT++ end with different final masses. There are no substantial changes, however, in the specific angular momentum content of the stellar cores. (A color version of this figure is available in the online journal.)

transport of angular momentum and chemicals by rotational instabilities and magnetic torques is included and calibrated following Heger et al. (2000, 2005) and Yoon \& Langer (2005). Wind mass loss is been implemented following the recipe of Glebbeek et al. (2009). For $T_{\text {eff }}>10^{4} \mathrm{~K}$ and $\mathrm{H}$-surface fraction $>0.4$, the mass-loss prescription of Vink et al. (2001) is used. In the same temperature range, but when the $\mathrm{H}$-surface fraction decreases below 0.4, Nugis \& Lamers (2000) determine the mass-loss rate. At low temperatures $\left(T_{\text {eff }}<10^{4} \mathrm{~K}\right)$ the massloss rate of de Jager et al. (1988) is used.

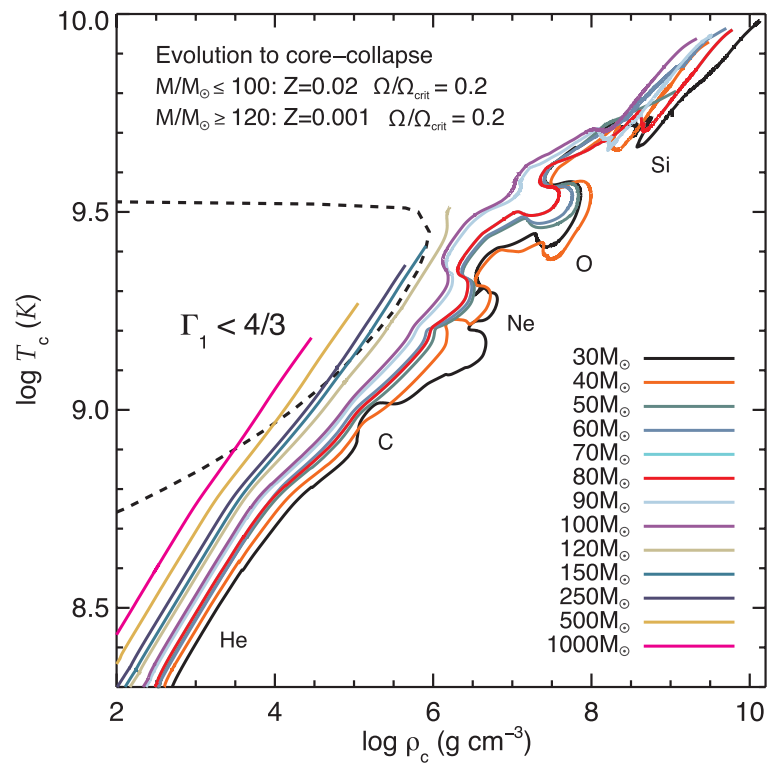

Figure 44. Evolution of $T_{\mathrm{c}}$ and $\rho_{\mathrm{c}}$ in the massive rotating models. The locations of core helium, carbon, neon, oxygen, and silicon burning are labeled. A dashed curve marks the electron-positron pair-instability region where $\Gamma_{1}<4 / 3$. All models are rotating initially at $20 \%$ of critical rotation. The calculations include the effects of rotation and Spruit-Tayler magnetic fields as discussed in Section 6. Models with initial mass $\leqslant 100 M_{\odot}$ have initial metallicity $Z=0.02$, while models with mass $\geqslant 120 M_{\odot}$ have initial metallicity $Z=0.001$. The end of the line for each mass corresponds to the time of core collapse, defined as when any part of the collapsing core exceeds an infall velocity of $1000 \mathrm{~km} \mathrm{~s}^{-1}$. The tracks for the $60 M_{\odot}$ and $70 M_{\odot}$ overlap in this plot.

(A color version of this figure is available in the online journal.)

Figure 44 shows the central conditions of these massive rotating models. For each model the calculation stops when any part of the collapsing core reaches an infall velocity of $1000 \mathrm{~km} \mathrm{~s}^{-1}$. Some of the initial and final properties are summarized in Table 5. These calculations are performed to reveal the new capabilities of MESAstar. The values of the 
Table 5

Initial (ZAMS) and Final (Core-collapse) Properties

\begin{tabular}{|c|c|c|c|c|c|c|c|c|c|c|c|}
\hline $\begin{array}{l}M_{\text {ini }} \\
\left(M_{\odot}\right)\end{array}$ & $Z_{\text {ini }}$ & $\Omega / \Omega_{\text {crit }^{\mathrm{a}}}$ & $\begin{array}{c}v_{\text {eq,ini }}{ }^{\mathrm{b}} \\
\left(\mathrm{km} \mathrm{s}^{-1}\right)\end{array}$ & $\begin{array}{c}J_{\text {ini }}{ }^{\mathrm{c}} \\
(\mathrm{erg} \mathrm{s})\end{array}$ & $\begin{array}{c}\Delta t^{\mathrm{d}} \\
(\mathrm{Myr})\end{array}$ & $\begin{array}{c}\Delta t_{\mathrm{H}}^{\mathrm{e}} \\
(\mathrm{Myr})\end{array}$ & $\begin{array}{l}\Delta t_{\mathrm{He}} \mathrm{e}^{\mathrm{e}} \\
(\mathrm{Myr})\end{array}$ & $\begin{array}{c}M_{\mathrm{f}}^{\mathrm{f}} \\
\left(M_{\odot}\right)\end{array}$ & $\begin{array}{l}M_{\mathrm{Fe}}^{\mathrm{g}} \\
\left(M_{\odot}\right)\end{array}$ & $\begin{array}{c}J_{\mathrm{f}}^{\mathrm{h}} \\
(\mathrm{erg} \mathrm{s})\end{array}$ & $\begin{array}{c}J_{\mathrm{Fe}}{ }^{\mathrm{i}} \\
(\mathrm{erg} \mathrm{s})\end{array}$ \\
\hline 30 & 0.020 & 0.20 & 129.69 & $3.28 \times 10^{52}$ & 6.30 & 5.87 & 0.36 & 17.77 & 1.41 & $2.87 \times 10^{50}$ & $1.03 \times 10^{48}$ \\
\hline 40 & 0.020 & 0.20 & 122.86 & $4.87 \times 10^{52}$ & 5.06 & 4.71 & 0.31 & 19.37 & 1.81 & $3.77 \times 10^{50}$ & $1.61 \times 10^{48}$ \\
\hline 50 & 0.020 & 0.20 & 112.02 & $6.30 \times 10^{52}$ & 4.41 & 4.08 & 0.29 & 25.04 & 1.38 & $5.39 \times 10^{50}$ & $1.09 \times 10^{48}$ \\
\hline 60 & 0.020 & 0.20 & 98.37 & $7.34 \times 10^{52}$ & 4.04 & 3.66 & 0.35 & 22.88 & 1.76 & $7.81 \times 10^{50}$ & $2.76 \times 10^{48}$ \\
\hline 70 & 0.020 & 0.20 & 78.76 & $7.53 \times 10^{52}$ & 3.90 & 3.57 & 0.29 & 26.19 & 1.75 & $5.30 \times 10^{50}$ & $1.54 \times 10^{48}$ \\
\hline 80 & 0.020 & 0.20 & 50.10 & $5.88 \times 10^{52}$ & 3.70 & 3.38 & 0.29 & 29.20 & 1.78 & $6.16 \times 10^{50}$ & $1.44 \times 10^{48}$ \\
\hline 90 & 0.020 & 0.20 & 2.27 & $3.57 \times 10^{52}$ & 3.10 & 2.80 & 0.27 & 44.90 & 1.71 & $4.39 \times 10^{50}$ & $5.23 \times 10^{47}$ \\
\hline 100 & 0.020 & 0.20 & 2.34 & $3.91 \times 10^{51}$ & 2.98 & 2.69 & 0.26 & 49.02 & 1.92 & $5.50 \times 10^{50}$ & $6.58 \times 10^{47}$ \\
\hline 120 & 0.001 & 0.20 & 145.41 & $2.93 \times 10^{53}$ & 3.26 & 2.99 & 0.23 & 79.38 & $\ldots$ & $4.79 \times 10^{51}$ & $\ldots$ \\
\hline 150 & 0.001 & 0.20 & 134.75 & $3.84 \times 10^{53}$ & 3.03 & 2.77 & 0.23 & 95.52 & $\ldots$ & $6.80 \times 10^{51}$ & $\ldots$ \\
\hline 250 & 0.001 & 0.20 & 69.30 & $4.39 \times 10^{53}$ & 2.56 & 2.32 & 0.21 & 167.49 & $\ldots$ & $9.13 \times 10^{51}$ & $\ldots$ \\
\hline 500 & 0.001 & 0.20 & 3.78 & $6.40 \times 10^{52}$ & 2.19 & 1.96 & 0.20 & 410.28 & $\ldots$ & $7.92 \times 10^{51}$ & $\ldots$ \\
\hline 1000 & 0.001 & 0.20 & 4.42 & $2.09 \times 10^{53}$ & 1.99 & 1.77 & 0.19 & 860.48 & $\ldots$ & $2.44 \times 10^{52}$ & $\ldots$ \\
\hline
\end{tabular}

Notes.

${ }^{a}$ Initial rotation rate; see definition in Section 6.4.

${ }^{\mathrm{b}}$ Initial equatorial rotational velocity.

c Total initial angular momentum.

d Stellar lifetime.

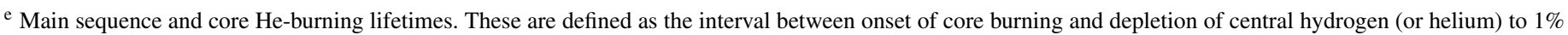
by mass.

${ }^{\mathrm{f}}$ Final mass.

g Mass of the iron core (if present).

${ }^{\mathrm{h}}$ Final total angular momentum.

${ }^{\mathrm{i}}$ Final total angular momentum of the iron-core.

parameters for these calculations have not been calibrated against existing calculations or observations.

\subsection{Radial Instability of Red Supergiants}

Massive RSGs are unstable to radial pulsations driven by the $\kappa$-mechanism in the hydrogen ionization zone. Both linear and nonlinear calculations show the occurrence of oscillations with the period and growth rate of the dominant fundamental mode increasing with $L / M$ (Li \& Gong 1994; Heger et al. 1997; Yoon \& Cantiello 2010). The periods are of the order of years. As discussed by Yoon \& Cantiello (2010) the occurrence of RSG pulsations can impact stellar mass-loss rates and modify the evolution of massive stars above a certain mass. We study the occurrence of RSG pulsations with MESAstar and compare results with existing nonlinear calculations.

In Figure 45 we show the capability of MESAstar to exhibit radial oscillations in luminous RSGs. We use the same $25 M_{\odot}$ rotating model discussed in Section 6.6, and we restart the calculation when the He mass fraction in the core is $Y_{c}=0.7$. For non-rotating RSG with $Z=0.02$, Yoon \& Cantiello (2010) found pulsation periods in the range $1-8 \mathrm{yr}$. To resolve the RSG pulsations we force the time step to $<0.01 \mathrm{yr}$, much shorter than the usual time step during He burning $\left(\delta t \gtrsim 10^{2} \mathrm{yr}\right.$; see Appendix B.3). This explains why RSG pulsations are usually not found during the evolution of massive stars. Before the code stops due to the emergence of supersonic radial velocities in the envelope, we find a pulsational period $\approx 4 \mathrm{yr}$, in good agreement with the results of Yoon \& Cantiello (2010).

\section{SUMMARY AND CONCLUSIONS}

We have explained and, where possible, verified the improvements and major new capabilities implemented in MESA since the publication of Paper I. These advancements include evolutionary modeling for giant planets (Section 2), tools for

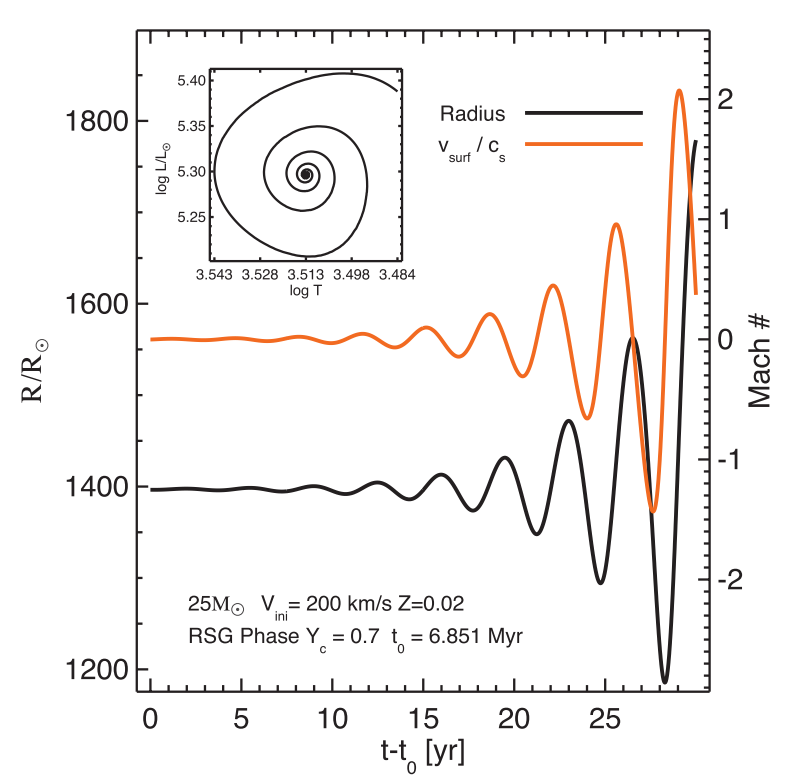

Figure 45. Surface properties of a pulsating RSG. This is the same $25 M_{\odot}$ model discussed in Section 6.6, evolved from $t_{0}=6.851 \mathrm{Myr}$ (corresponding to $Y_{c}=0.7$, star symbol in Figure 32 ) with time steps $\delta t \leqslant 0.01 \mathrm{yr}$. The black line shows the evolution of the stellar radius, while the orange line shows the value of the surface radial velocity (in units of the local sound speed). The inset shows the corresponding evolution in the H-R diagram.

(A color version of this figure is available in the online journal.)

asteroseismology (Section 3), implementation of composition gradients in stellar interiors and their impact on convective mixing (Section 4), the evolution of intermediate mass stars and WDs (Section 5) the treatment of rotation during stellar evolution (Section 6), addressing the onset of radiation pressure dominance in the envelopes of evolving massive stars due to the iron opacity bump, and evolving massive stars to the on- 
set of core collapse (Section 7). The enhancements include the physics modules (Appendix A), the algorithms (Appendix B), and the addition of a MESA SDK (Appendix C). MESAstar input files and related materials for all the figures are available at http://mesastar.org.

These hitherto unpublished advancements have already enabled a number of studies in planets (e.g., Passy et al. 2012; Huang \& Cumming 2012; Carlberg et al. 2012), CNe (Denissenkov et al. 2013), asteroseismology (e.g., Yang et al. 2012; Burkart et al. 2012; Moravveji et al. 2012), rotationally induced mixing (e.g., Denissenkov 2010; Chatzopoulos et al. 2012; Chatzopoulos \& Wheeler 2012) and enabled the discovery of new features in the evolution of low-mass stars (Denissenkov 2012). In addition, these enhanced capabilities have allowed for applications of MESAstar that were not initially envisioned, such as explorations of stars under modified gravity (Chang \& Hui 2011; Davis et al. 2012) and stellar oscillations induced by tidal disturbances in double WD binaries (Fuller \& Lai 2012, 2013; Burkart et al. 2013).

As an open source "instrument" for stellar astrophysics, it is difficult to predict all the ways in which future development of MESA will occur. We do know, however, that future versions of MESA will include advances in physics modules, features driven by the MESA user community, and architectural refinements. For example, the plethora of asteroseismological data is driving new initiatives to incorporate non-adiabatic pulsation codes, where possible, into MESA. The prevalence of interacting binary star systems, especially for massive stars, has increased the pressure for MESA development efforts that would yield the capability to simultaneously evolve two interacting stellar models. Physics module developments will likely include general relativistic corrections to the stellar structure equations (e.g., difference between gravitational and baryonic mass), the mass diffusion coefficients in electron degenerate environments, phase separation in cooling WDs, and nuclear statistical equilibrium solvers. We also expect the transition from multicore systems (with order 10 cores) to many-core architectures (with order 100 cores) to drive new directions in MESA's algorithmic and architectural development.

It is a pleasure to thank Falk Herwig for significant contributions to the MESA project and Jørgen Christensen-Dalsgaard for kindly providing the ADIPLS code for inclusion in MESA and assisting with its integration. We likewise thank Jared Brooks for documenting the MESA test suite; Tristan Guillot for providing the CEPAM evolutionary tracks; Alexander Potekhin for an update to his EOS code; Didier Saumon and Jim MacDonald for providing EOS tables; René Rohrman for providing atmosphere tables; Richard Freedman for providing opacity tables; Evert Glebbeek, Alex Heger, and Norbert Langer for providing code for implementing rotation; and Haili $\mathrm{Hu}$ for providing code for implementing diffusion.

We also thank David Arnett, Tim Bedding, Kent Budge, Phil Chang, Pieter DeGroote, Pavel Denisenkov, Jonathan Fortney, Chris Fryer, Gustavo Hime, Raphael Hirschi, Sam Jones, Steve Kawaler, Phillip Macias, Pablo Marchant, Travis Metcalfe, Kevin Moore, Ehsan Moravveji, Jean-Claude Passy, Hideyuki Saio, Josiah Schwab, Aldo Serenelli, Josh Shiode, Steinn Sigurdsson, Anne Thoul, Roni Waldman, Achim Weiss, Stan Woosley, Sung-Chul Yoon, and Patrick Young for providing valuable discussions and correspondence. Some of the simulations for this work were made possible by the Triton Resource, a high performance research computing system operated by San Diego Supercomputer Center at UC San Diego.

We thank the participants of the 2012 MESA Summer School for their willingness to experiment with the new capabilities: Jeff Andrews, Umberto Battino, Keaton Bell, Harshal Bhadkamkar, Kristen Boydstun, Emmanouil Chatzopoulos, Eugene Chen, Jieun Choi, Alex Deibel, Luc Dessart, Ian Dobbs-Dixon, Tassos Fragos, Samuel Harrold, Daniel Huber, Joe Hughto, Max Katz, Agnes Kim, Io Kleiser, Shri Kulkarni, Gongjie Li, Christopher Lindner, Jing Luan, Mia Lundkvist, Morgan MacLeod, João Marques, Grant Newsham, Rachel Olson, Richard O'Shaughnessy, Kuo-Chuan Pan, Ilka Petermann, Theodore Sande, Ken Shen, Natalia Shabaltas, Dave Spiegel, Jie Su, Tuguldur Sukhbold, David Tsang, Bill Wolf, Angie Wolfgang, Tsing Wai Wong, and Alexey Zinger.

This project was broadly supported by the NSF under grants PHY 11-25915 and AST 11-09174. M.C. acknowledges partial support from the "Alberto Barlettani" Prize 2012. P.A. acknowledges support by NSF AST-0908873 and NASA NNX09AF98G. L.B. acknowledges support from the Wayne Rosing, Simon and Diana Raab Chair in Theoretical Astrophysics at KITP. E.F.B acknowledges support by the Joint Institute for Nuclear Astrophysics under NSF PHY grant 08-22648. A.L.D received support from the Australian Research Council under grant FL110100012. M.H.M acknowledges support by the NSF Grant AST-0909107, the NASA Grant NNX12AC96G, the Norman Hackerman Advanced Research Program under grant 003658-0252-2009, and the Delaware Asteroseismic Research Center. D.S. acknowledges support by the Australian Research Council. F.X.T acknowledges support from the NSF under grants AST 08-06720, AST 6736821, AST 09-07919, AST 10-07977, PHY 08-22648, and from NASA under grants 08-NAI5-0018 and NNX11AD31G. R.T. acknowledges support by NSF Grants AST-0908688 and AST-0904607, and NASA Grant NNX12AC72G.

\section{APPENDIX A \\ UPDATES TO INPUT PHYSICS MODULES}

There have been many updates and improvements to the physics modules since Paper I. In this Appendix, we describe the changes that have been made to the microphysics modules chem (Appendix A.1), eos (Appendix A.2), kap (Appendix A.3), and net (Appendix A.4). We conclude by listing updates to the atmosphere boundary conditions (Appendix A.5).

\section{A.1. Atomic and Nuclear Data}

The chem module now has the latest version (v2.0) of the JINA reaclib nuclide data (Cyburt et al. 2010). This contains updated mass evaluations, and now includes 7853 nuclides up to ${ }^{337} \mathrm{Cn}$. For precision work, the chem module now distinguishes between the atomic mass number $A_{i}$ - the number of nucleons in a given isotope-and the atomic mass $W_{i}$. The abundance of a species $i$ is defined as

$$
Y_{i} \equiv \frac{n_{i}}{n_{\mathrm{B}}}
$$

where $n_{\mathrm{B}}$ is the baryonic number density. The baryon fraction $X_{i}$ is then

$$
X_{i}=Y_{i} A_{i}=\frac{n_{i} A_{i}}{n_{\mathrm{B}}},
$$


Note that $\sum_{i} X_{i}=n_{\mathrm{B}} / n_{\mathrm{B}}=1$ and is invariant under nuclear reactions. We then define the baryon density (in mass units) as

$$
\rho=n_{\mathrm{B}} m_{\mathrm{u}}
$$

where $m_{\mathrm{u}}=1.660538782 \times 10^{-24} \mathrm{~g}$ is the atomic mass unit (CODATA 2006 value; Mohr et al. 2008). Note that the numerical value $m_{\mathrm{u}}$, along with other physics constants, are defined in the const module. The atomic mass of isotope $i$ is defined in MESA as

$$
W_{i}=A_{i}+\frac{\Delta_{i}}{m_{\mathrm{u}} c^{2}},
$$

where $\Delta_{i} / c^{2}$ is the mass excess of isotope $i$. This treatment neglects the electronic binding energy, and $\Delta$ is therefore independent of the ionization state of a given species. The electron rest masses are, however, included in this definition, since the $W_{i}$ are atomic masses.

The MESA microphysics modules-kap, eos, neu, and net-use $\rho, T$, and $\left\{X_{i}\right\}$ as inputs. MESAstar multiplies $\rho$ by a mass correction factor $\bar{W} / \bar{A}=\sum_{i} W_{i} Y_{i} / \sum_{i} A_{i} Y_{i}$ to distinguishes between $A_{i}$ and $W_{i}$ before starting the calculation for a time step. A call to the routine composition_info in the chem module returns the following averaged quantities: the mean atomic mass number, $\bar{A} \equiv \sum_{i} Y_{i} A_{i} / \sum_{i} Y_{i}$, mean atomic charge number, $\bar{Z} \equiv \sum_{i} Z_{i} Y_{i} / \sum_{i} Y_{i}$, mean square atomic charge number, $\sum_{i} Z_{i}^{2} Y_{i} / \sum_{i} Y_{i}$, the electron abundance, $Y_{e}=\bar{Z} / \bar{A}$, and the mass correction term, $\bar{W} / \bar{A}$. In addition, the routine returns the derivatives of $\bar{A}, \bar{Z}$, and $\bar{W} / \bar{A}$ with respect to the baryon fractions $X_{i}$ :

$$
\begin{aligned}
\left.\frac{\partial \bar{A}}{\partial X_{i}}\right|_{\rho, X_{j \neq i}} & =\frac{\bar{A}}{A_{i}}\left(A_{i}-\bar{A}\right) \frac{1}{\sum_{i} X_{i}} ; \\
\left.\frac{\partial \bar{Z}}{\partial X_{i}}\right|_{\rho, X_{j \neq i}} & =\frac{\bar{A}}{A_{i}}\left(Z_{i}-\bar{Z}\right) \frac{1}{\sum_{i} X_{i}} ; \\
\left.\frac{\partial(\bar{W} / \bar{A})}{\partial X_{i}}\right|_{\rho, X_{j \neq i}} & =\left(\frac{W_{i}}{A_{i}}-\frac{\bar{W}}{\bar{A}}\right) \frac{1}{\sum_{i} X_{i}} .
\end{aligned}
$$

Note that the routine does not make any assumption in these derivatives that $\sum_{i} X_{i} \equiv \sum_{i} A_{i} Y_{i}=1$; in this formulation, $\sum_{i} X_{i}$ is not explicitly set to unity.

At the beginning of each Newton iteration, the abundances are checked. A mass fraction is considered good if its value exceeds min_xa_hard_limit. If all mass fractions meet this standard, then the mass fractions are clipped to range from 0 to 1 , and the mass fractions are summed. If the sum differs from unity by less than a value sum_xa_tolerance, then the mass fractions are renormalized to sum to unity; otherwise, the code reports an error. Currently composition derivatives are ignored in the eos and kap routines. Equations (A5)-(A7) allow, however, future additions to these routines to compute these derivatives analytically.

\section{A.2. Equation of State}

The only significant change to the eos module since Paper I is the addition of tables for $Z>0.04$, where $Z$ is the mass fraction of all elements heavier than He. The eos module as described in Paper I supplied EOS tables for $Z=0.0,0.02$, and 0.04 at temperatures and densities for which neutral and partially ionized species are present (see Paper I, Figure 1). For $Z>0.04$ MESA switched to the HELM EOS (Timmes \& Swesty 2000), which assumes full ionization. In order to rectify the inconsistent treatment of the partially ionized region at high $Z$, new EOS tables have been computed (J. MacDonald 2012, private communication) using the MacDonald EOS code (MacDonald \& Mullan 2012) for $Z=0.2$ (scaled-solar), and two $Z=1.0$ compositions: one with $49.5 \% \mathrm{C}, 49.5 \% \mathrm{O}$, and $1 \%$ scaled-solar by mass; and one with $50 \% \mathrm{C}$ and $50 \% \mathrm{O}$ by mass. Here "scaled-solar" refers to the Grevesse \& Noels (1993) solar heavy element distribution adopted in the OPAL EOS tables (Rogers \& Nayfonov 2002).

\section{A.3. Opacities}

The kap module now divides the opacity tables into a hightemperature domain, $\log (T / \mathrm{K}) \gtrsim 4$, and a low-temperature domain, $\log (T / \mathrm{K}) \lesssim 4$; the exact range of $\log T$ over which the tables are blended can be adjusted at runtime. This treatment differs from the opacity tables described in Paper I, which combined high- and low-temperature opacities into a single set of tables. The motivation for separating the tables is to facilitate using different sources of low- $T$ opacity data. The kap module now supports low- $T$ opacities from either Ferguson et al. (2005) or Freedman et al. (2008) with updates to the molecular hydrogen pressure-induced opacity (Frommhold et al. 2010) and the ammonia opacity (Yurchenko et al. 2011). Either set may be selected at run time. The electron conduction opacity tables, based on Cassisi et al. (2007), have been expanded (A. Y. Potekhin 2011, private communication) to cover higher temperatures (up to $10^{10} \mathrm{~K}$, originally $10^{9} \mathrm{~K}$ ) and densities (up to $10^{11.5} \mathrm{~g} \mathrm{~cm}^{-3}$, originally $10^{9.75} \mathrm{~g} \mathrm{~cm}^{-3}$ ).

\section{A.4. Nuclear Reactions}

Substantial improvements to the net module have been made since Paper I to increase the flexibility of the nuclear reaction networks (see Appendix B for working details). One such improvement is the standalone one-zone burn routines. These now operate on a user-defined initial composition, nuclear network, and a thermodynamic trajectory. Choices for the thermodynamic trajectory include a burn with density and temperature held fixed, a burn with pressure held fixed, and a burn with the density and temperature following an arbitrary, user-specified profile. This last option is activated by setting read_T_Rho_history=.true. and specifying the file name containing the profile through the variable T_Rho_history_filename. The MESA one-zone burn routines now include user-specified options for the family of stiff ordinary differential equation integrators from Hairer \& Wanner (1996). In addition, three user-defined switches are provided to switch between using dense matrix linear algebra solvers, for smaller networks, and sparse matrix linear algebra solvers for larger ones. The option decsol_switch sets the number of isotopes at which the switch occurs; options small_mtx_decsol and large_mtx_decsol specify the dense and sparse solvers, respectively.

Figure 46 shows the constant pressure option of these routines operating on conditions that might be encountered for helium burning on the surface of a WD. The initial pressure is $3.1 \times$ $10^{22} \mathrm{erg} \mathrm{cm}^{-1}$, the initial temperature is $2 \times 10^{8} \mathrm{~K}$, the initial composition is $X\left({ }^{4} \mathrm{He}\right)=0.98$ and $X\left({ }^{14} \mathrm{~N}\right)=0.02$, and 


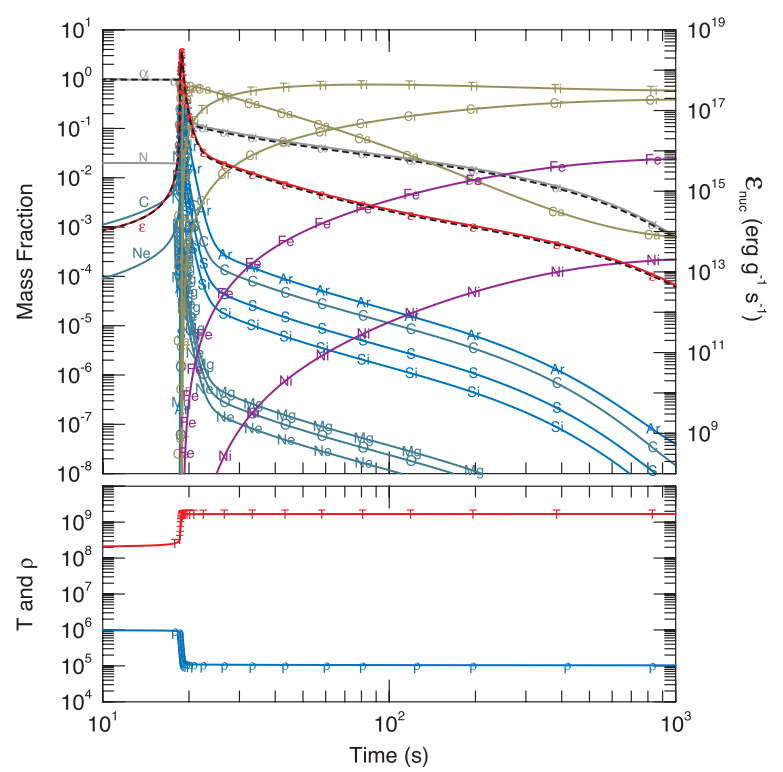

Figure 46. One-zone helium and nitrogen burn at constant pressure, $P=$ $3.1 \times 10^{22} \mathrm{erg} \mathrm{cm}^{-3}$, starting from an initial temperature of $T=2 \times 10^{8} \mathrm{~K}$. Evolution of the temperature and density are shown in the lower panel, while the upper panel shows the mass fraction of key isotopes (right axis) and the energy generation rate per unit mass (left axis; red curve). MESA results are shown by the colored and labeled curves, and the results from an independent one-zone burner (Timmes 1999) are shown by the dashed black curves.

(A color version of this figure is available in the online journal.)

the system was evolved for $10^{4} \mathrm{~s}$ with a 19 -isotope network. Evolution of the density and temperature under the constant burn conditions are shown in the lower panel of Figure 46. The temperature slowly increases and the density slowly decreases as the material begins to burn and release energy at a rate of $\epsilon_{\text {nuc }}=$ $c_{P} d T / d t$. When the temperature crosses a critical threshold at $\approx 20 \mathrm{~s}$, a runaway occurs as the temperature rapidly rises and the composition burns to heavier elements. The material then establishes a final equilibrium state, no energy from nuclear burning is injected into the system, and the temperature reaches a plateau.

The upper panel of Figure 46 compares the evolution of key isotopes and the energy generation rate per unit mass of the MESA one-zone burner (colored and labeled curves) with an independent one-zone burner (dashed black curves) based on Timmes (1999). These comparisons indicate that both onezone burns produce a final composition that is mostly ${ }^{44} \mathrm{Ti}$ and ${ }^{48} \mathrm{Cr}$. Over most of the evolution, the two calculations give mass fractions of various isotopes that agree to within 2-3 significant digits. Larger differences in some of the heavier isotopes at the end of the calculation are due to differences in the adopted nuclear reaction rates.

Another improvement is the net module now accesses reactions from both weaklib and reaclib. Rather than evaluating the standard seven-parameter fit for $N_{\mathrm{A}}\langle\sigma v\rangle$ for the reaclib rates (Cyburt et al. 2010) every time a reaction rate is needed, the net module caches separate rate tables for each reaction. Inverse rates are calculated directly from the forward rates (those with positive $Q$-value) using detailed balance, rather than fitted, rates. This is important for explosive nucleosynthesis approaching nuclear statistical equilibrium (see Calder et al. 2007). The nuclear partition functions used to calculate the inverse rates are taken from Rauscher \& Thielemann (2000).

\section{A.5. Atmosphere Boundary Conditions}

The atm module provides the surface boundary condition for the interior model. A collection of four new options that extend the set described in Paper I are now available.

1. solar_Hopf_gray. Implements the solar-calibrated Hopf $T(\tau)$ relation, where

$$
T^{4}(\tau)=\frac{3}{4} T_{\mathrm{eff}}^{4}[\tau+q(\tau)]
$$

and

$$
q(\tau)=q_{1}+q_{2} \exp \left(-q_{3} \tau\right)+q_{4} \exp \left(-q_{5} \tau\right) .
$$

The $q_{i}$ are fit to the solar atmosphere with resulting values $q_{1}=1.0361, q_{2}=-0.3134, q_{3}=2.448, q_{4}=-0.2959$, and $q_{5}=30.0$ (J. Christensen-Dalsgaard 2011, private communication).

2. gray_and_kap. Expands on the simple assumption that $P \simeq \tau g / \kappa$ by iterating to find a consistent solution among $P, T$, and $\kappa(\rho, T)$.

3. gray_irradiated. Implements the Guillot (2010) $T(\tau)$ relation that includes both external irradiation by the star and cooling flux from the interior; see Guillot (2010, Equation (49)) along with the discussion and results in Section 2.4. In addition to the external and internal fluxes, this boundary condition requires two constant opacity values: $\kappa_{\mathrm{v}}$ for the external radiation, and $\kappa_{\mathrm{th}}$ for the thermal radiation generated within the atmosphere. This boundary condition is unique in that it is applied at a specified pressure level, as opposed to optical depth. This pressure must be chosen sufficiently high to capture any heating of the atmosphere by the irradiation.

4. WD_tau_25_tables. Provides as outer boundary conditions the values of $P_{\text {gas }}$ and $T$ at $\log (\tau)=1.4$ as extracted from pure hydrogen model atmospheres of WDs (Rohrmann et al. 2012; Rohrmann 2001). The tables span a range of effective temperatures and surface gravities: $2000 \mathrm{~K} \leqslant T_{\text {eff }} \leqslant 40,000 \mathrm{~K}$ and $5.5 \leqslant \log g \leqslant 9.5$. See Section 5.2 for an example of the use of these tables.

\section{APPENDIX B}

\section{NUTS AND BOLTS}

We now briefly describe the primary components of evolution calculations. MESAstar first reads the input files and initializes the physics modules to create a nuclear reaction network and access the EOS and opacity data. The specified starting model is then loaded into memory and the evolution loop is entered.

\section{B.1. Evolve a Step}

The top level routine for evolving a star for a single time step is do_evolve_step. If this is the first attempt to do a step starting from the current state, the model is remeshed (see Appendix B.4), and information for MLT++ is prepared by the routine set_gradT_excess_alpha (see Section 7.2). Sufficient information is saved so that if necessary it will be possible to make other attempts (i.e., after a redo, a retry, or a backup). In addition to the current state, we keep the previous state (called "old"), and the one that came before "old" (called "older"). During the step, the current state is modified, and the old one holds the state at the start of the step. If we do a redo or a retry, we copy 


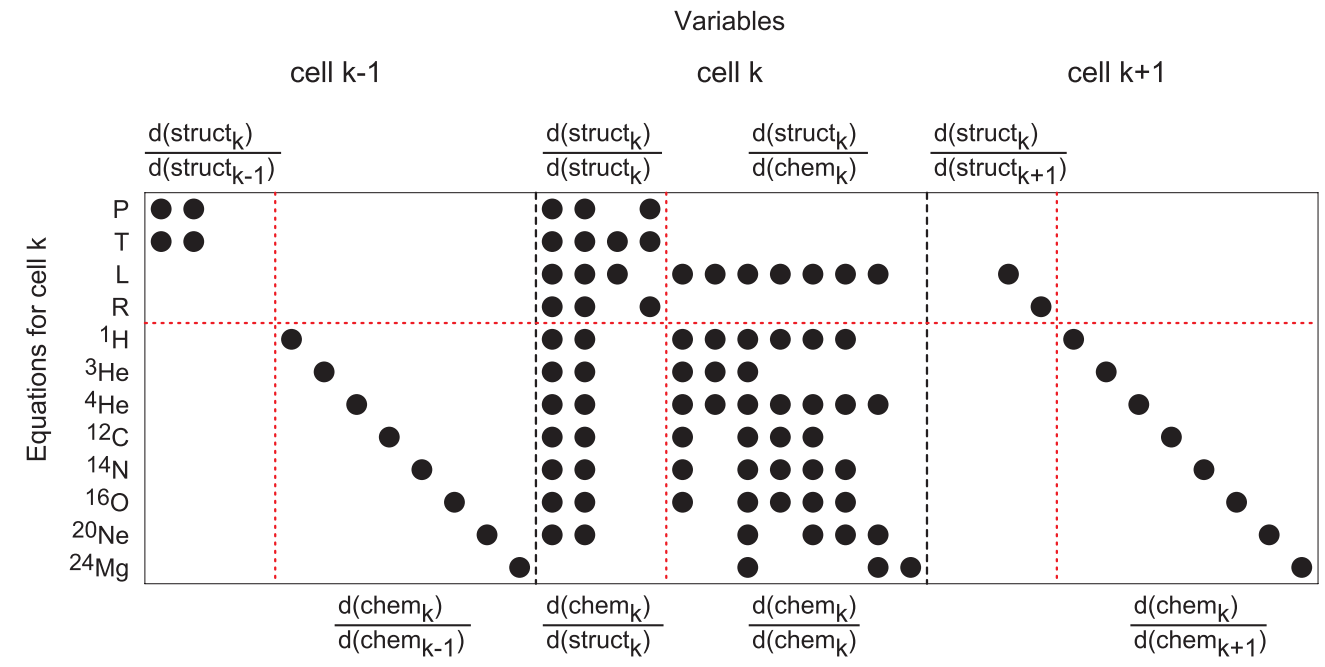

Figure 47. One row of the block tridiagonal Jacobian matrix for a $2.5 M_{\odot}$ ZAMS model, with black dots showing the locations of non-zero entries.

(A color version of this figure is available in the online journal.)

old to current to restore the starting state. If we do a backup, we copy older to old before copying old to current, making us start at the state prior to the current one. Note that the duration of the time step is determined before the call on do_evolve_step by the process described in Appendix B.3.

After remeshing and the other initial preparations, do_evolve_step begins the operations that are done on every attempt. It first calls the routine do_winds which sets $\dot{M}$ based on the current radius, luminosity, mass, metallicity, and other properties as needed. During the evaluation of do_winds there is a call on the user-defined other_wind routine giving users an easy way to define different schemes for setting $\dot{M}$.

Information for evaluating the Lagrangian time derivatives is stored by a call to save_for_d_dt. The ensuing call to do_adjust_mass adds or removes mass without changing the number of grid points (see Appendix B.5). Information for evaluating the Lagrangian time derivatives is updated at this point. Variables for the model are evaluated to reflect the changes made by remeshing and changing mass. This includes evaluation of the Brunt-Väisälä frequency (see Section 3.3), and the diffusion coefficients for the mixing of composition (see Sections 4.1 and 4.2). The user-defined routines other_brunt, other_mlt, and other_mixing are called as part of this. If rotation is enabled, there is a call to set_rotation_mixing_info (see Section 6) which in turn calls other_am_mixing. If element diffusion from gravitational settling and chemical diffusion is active, the routine do_element_diffusion adjusts the composition and includes a call on other_diffusion The ensuing call to do_struct_burn_mix solves for the new structure and composition of the star through repeated Newton iterations (see Appendix B.2). Non-convergence causes do_evolve_step to return with a result indicating a failure. Convergence is followed by a call to the routine do_solve_omega_mix which adjusts the total angular momentum by solving a diffusion equation (see Appendix B.6); it calls other_torque. There is an option to repeat the operations described in this paragraph in case rotationally enhanced mass loss (see Section 6.4) has not been sufficient to eliminate super-critical surface velocities. In such a situation, the mass loss is adjusted iteratively until slightly subcritical velocities result. In effect, this is an implicit solution for the appropriate $\dot{M}$ when super-critical rotation occurs.
Next, if specified by the user, smooth_convective_bdy is called to smooth abundances behind retreating convection boundaries. Finally, a call to do_report gathers information and metrics about the time step for the user. This information will then be available to the user's extras_check_model routine.

\section{B.2. Solving the Coupled Structure, Burn, and Mix Equations}

A call to do_struct_burn_mix invokes a Newton method-an $\mathrm{N}$-dimensional root find-to solve a system of $N$ nonlinear differential-algebraic equations for the new structure and composition of the stellar model. Here $N$ is the number of zones in the current model times the number of basic variables per zone and can exceed 100,000.

The equations to be solved are written as the relation $F$ (basic_vars) $=0$, where $F$ is the vector-valued function of the residuals. If the basic_vars were a perfect solution to the equations, we would have $F=0$; in practice, the solution is never perfect. The solution strategy is to iteratively adjust the values of the basic_vars to reduce $F$ toward zero. An approximate solution is accepted depending on both the magnitude of $F$ and the relative size of the adjustments to basic_vars. Adjustments are chosen using the Jacobian matrix of partial derivatives of all the $F$ equations with respect to all the basic_vars.

Figure 47 shows the three blocks making up the row of the block tridiagonal Jacobian matrix for the tenth from the center cell of a non-rotating $2.5 M_{\odot}$ ZAMS model, with black dots showing non-zero entries. The partial derivatives of the equations for cell $k$ form the rows of the blocks. In this case, we have four equations for the structure of the model $(P, T, L$, and $r)$ and eight equations for the chemical abundances $\left({ }^{1} \mathrm{H}\right.$ through ${ }^{24} \mathrm{Mg}$ ). Each block of the tridiagonal matrix is demarcated by dashed black vertical lines. The block matrix on the left shows the dependencies of the equations for cell $k$ on the variables of cell $k-1$, the one in the middle shows the dependencies of the equations for cell $k$ on the variables of cell $k$, and the one on the right shows the dependencies of the equations for cell $k$ on the variables of cell $k+1$. The dashed lines partition each block into four sub-blocks to highlight the structure and abundance portions of each block. 
The structure of the lower-right subblocks in the left and right blocks shows that the chemical abundance of a particular species in cell $k$ depends on the chemical abundances of that species in cells $k-1$ and $k+1$; this is because of mixing between neighboring cells. In this specific case of a non-rotating $2.5 M_{\odot}$ ZAMS model the mixing of chemical elements between cells is only due to the treatment of convection. The lower-right subblock of the center block also shows the interdependencies of abundances due to nuclear reactions in the cell. The bottomleft subblocks are zero in the left and right blocks but show dependencies on the $P$ and $T$ variables of the center block. This is because the nuclear reactions that change the abundances depend of $P$ and $T$ of that cell but do not depend on $P$ and $T$ in the neighboring cells. The columns for $L$ and $r$ are zero in the center lower-left subblock because the equations for the abundances do not directly depend on those variables. The upper-right subblocks are zero in the left and right blocks but show that the equation for $L$ depends on the abundance variables in the center block. This is because the $L$ equation includes results from nuclear burning, and that depends directly on the composition of cell $k$ but not on the composition in neighboring cells. The other rows in the center upper-right subblock are zero because the equations for $P, T$, and $r$ do not directly depend on composition.

Finally, consider the upper-left subblocks that show the dependencies of structure equations on structure variables. The upper-left subblock in the center shows that each structure equation in $k$ depends on three or four of the structure variables in $k$. The $P$ and $T$ equations for cell $k$ also depend on both of the variables $P$ and $T$ in $k-1$, while the $L$ and $r$ equations for $k$ depend on the corresponding variables in $k-1$. This pattern reflects the form of the finite differences in the implementation of the structure equations: $P$ and $T$ differences use the outer neighbor $(k-1)$ while $L$ and $r$ differences use the inner neighbor $(k+1)$. The $L$ and $r$ equations for innermost cell $k=n$ use $L_{\text {center }}$ and $R_{\text {center }}$; the $P$ and $T$ equations for the outermost cell $k=1$ use the surface boundary conditions.

The structure variables for each zone always include the zone average of the natural logarithm of the temperature, $\ln T$, the luminosity at the outer edge of the zone, $L$, the natural logarithm of the radius at the outer edge of the zone, $\ln r$, and a second thermodynamic variable-either the zone average of the natural logarithm of the mass density, $\ln \rho$, or the zone average of the natural logarithm of the gas pressure, $\ln P_{\text {gas }}$. Ideally it would not matter whether $\ln \rho$ or $\ln P_{\text {gas }}$ was used as the second thermodynamic variable-for a given temperature and composition the EOS permits going back and forth between the two. Microphysics packages tend to use mass density as a primary input (i.e., they use a Helmholtz free energy basis), leading to the common choice of $\ln \rho$. However, the structure equations are solved only to within a finite but nonzero residual (see above). Approximately correct values for the density and temperature can then lead to anomalous pressure profiles, with tiny violations of hydrostatic balance. These local violations tend to appear near large jumps in density, such as at a sharp $\mathrm{H} / \mathrm{He}$ boundary. Using $P_{\text {gas }}$ as the second thermodynamic variable (effectively using a Gibbs free energy basis) removes these anomalous pressure profiles. For example, in stellar models without overshooting or semiconvection, the $\mathrm{H} / \mathrm{He}$ boundary is extremely sharp. Using the gas pressure as the second thermodynamic variable results in single zone step function transitions in the abundances and in the density, while the temperature and pressure are smooth across the transition.
Applications that demand smooth pressure profiles, such as pulsation analysis (see Section 3), should generally specify the gas pressure as the second thermodynamic variable.

MESAstar treats convective mixing as a time-dependent, diffusive process with a diffusion coefficient, $D$, determined by the MLT module. See Paper I for the implementation details of standard mixing length treatment. In addition to this standard MLT treatment, the MLT module includes the option to use the modified MLT++ prescription described in Sections 7.1 and 7.2. After the convective mixing calculations have been performed, MESAstar calculates the overshoot mixing diffusion coefficient as described in Paper I. During the solution of the coupled structure, burning, and mixing equations the equation for mass fraction $X_{i, k}$ of species $i$ in cell $k$ is determined by

$$
\begin{aligned}
X_{i, k}(t+\delta t)-X_{i, k}(t) & =d X_{\text {burn }}+d X_{\text {mix }} \\
& =\frac{d X_{i, k}}{d t} \delta t+\left(F_{i, k+1}-F_{i, k}\right) \frac{\delta t}{d m_{k}},
\end{aligned}
$$

where $d X_{i, k} / d t$ is the rate of change from nuclear reactions, $F_{i, k}$ is the mass of species $i$ flowing across face $k$

$$
F_{i, k}=\left(X_{i, k}-X_{i, k-1}\right) \frac{\sigma_{k}}{\overline{d m}_{k}},
$$

where $\sigma_{k}$ is the Lagrangian diffusion coefficient from the combined effects of convection and overshoot mixing and $\overline{d m}_{k}=$ $0.5\left(d m_{k-1}+d m_{k}\right)$. For numerical stability, $\sigma_{k}$ is calculated at the beginning of the time step and held constant during the implicit solver iterations. This assumption accommodates the non-local overshooting algorithm and significantly improves the numerical convergence. The structure of the lower-right subblocks in the left and right blocks in Figure 47 shows that the dependency of the chemical abundances in cell $k$ depends on the chemical abundances of that species in cells $k-1$ and $k+1$ as a result of convective mixing.

If the optional hydrodynamic mode is activated, then the radial velocity at the outer edge of the cell, $v$, is added to the structure variables. Figure 47 shows the order of the model variables in the Jacobian: each cell includes the structure variables followed by the mass fraction $X_{i}$ of each isotope. Mass and the local angular velocity $\omega$ are not treated as structure variables because they are held constant during the Newton iterations. The mass is set before the iterations, while $\omega$ is determined after convergence. This is computed taking into account loss/gain of angular momentum during the time step, the new stellar structure and internal transport of angular momentum calculated by a diffusion equation (see Appendix B.6).

The program flow to solve the coupled structure, burning, and mixing equations is to first create the matrix of partial derivatives using the current candidate solution, solve the block tridiagonal system of linear equations for the corrections to the basic variables, apply the possibly damped corrections (see next paragraph) to update the candidate solution, and calculate the residual $F$. Then, if the residual is small enough, we declare victory, otherwise we repeat the general flow with the updated candidate solution.

Each iteration of the Newton solver uses a linear approximation to create a vector of corrections to the model. These corrections do not include the physical requirement that the abundance mass fractions need to remain positive. To reduce the possible occurrence of negative abundances MESAstar now 
uses a damped Newton scheme. This checks for proposed corrections that would produce negative abundances and multiplies the entire correction vector by a factor less than one, so that only part of the full correction is applied. In many cases, this is sufficient to significantly improve the convergence properties of a model. In other cases, the damped correction scheme may force so many small corrections that the Newton solver cannot converge within the user-specified maximum number of iterations, forcing the previous model to be attempted again with a smaller time step (termed "a backup"). On balance, this is usually a small price to pay for improved conservation of species and more accurate solutions.

The modules in star provide routines to evaluate the residual equations and create the Jacobian matrix. Given a candidate solution (i.e., the set of basic variables for each cell), the microphysics for each cell (EOS, thermal neutrino loss, opacity, nuclear reaction rates) are evaluated in parallel (see Appendix B.9). The Jacobian matrix is then further populated with elements from rotation, artificial viscosity, and MLT for the temperature gradient, and these are also evaluated in parallel. Each of the routines that evaluate these components returns output values and partial derivatives of the output values with respect to the input values. Analytic partial derivatives are used whenever feasible, otherwise numerical partial derivatives are supplied.

\section{B.3. Time Step Controls}

Control of the time step is a critical part of stellar evolution and requires careful trade offs. The time steps must be small enough to allow convergence in comparatively few iterations but large enough to allow sufficiently efficient evolutions. Changes to the time step must respond rapidly to varying structure or composition conditions, but they need to be controlled to avoid large jumps that can reduce the convergence rate or the accuracy of the results. The routine pick_next_time step performs time step control as a two-stage process. The first stage proposes a new time step using the H211B low-pass filter (Söderlind \& Wang 2006), a scheme based on digital control theory. The second stage implements a wide range of tests that can reduce the proposed time step if certain selected properties of the model are changing too much in a single time step.

For the first stage, routine hydro_time step sets the variable for the next time step, dt_next, according to the relative magnitude of changes to the basic_vars. The variable reflecting the size of these changes is called varcontrol and is calculated by the routine eval_varcontrol. For improved stability and response, the low-pass controller uses previous and current values of varcontrol to make the next time step match the varcontrol_target, $w_{t}$, which is $10^{-4}$ by default. To make this explicit, let $\delta t_{i-1}, \delta t_{i}$, and $\delta t_{i+1}$ be the previous, current, and next time step, respectively, while $w_{c, i-1}$ and $w_{c, i}$ are the previous and current values of varcontrol. The maximum time step for model $i+1$ is then determined by

$$
\delta t_{i+1}=\delta t_{i} f\left[\frac{f\left(w_{t} / w_{c, i}\right) f\left(w_{t} / w_{c, i-1}\right)}{f\left(\delta t_{i} / \delta t_{i-1}\right)}\right]^{1 / 4},
$$

where $f(x)=1+2 \tan ^{-1}[0.5(x-1)]$. This control scheme allows rapid changes in the time step without undesirable fluctuations.

The time step proposed by this low-pass filtering scheme can be reduced in the second stage according to a variety of special tests that have hard and soft limits. If a change exceeds its specified hard limit, the current trial solution for the new step is rejected, and the code is forced to do a retry or a backup. If a change exceeds its specified soft limit, the next time step is reduced proportionally. The current classes of special cases that can reduce the next time step are limits based on:

1. Number of Newton iterations required to converge.

2. Maximum absolute change in the mass fraction of hydrogen or helium in any cell.

3. Maximum relative change in any mass fraction at any cell.

4. Magnitude in the relative change in the structure variables in each cell.

5. Nuclear energy generated in each cell for several categories of nuclear reactions.

6. Changes in the luminosity resulting from nuclear burning.

7. Changes at the photosphere in $\ln L$ and $T_{\text {eff }}$.

8. Changes in $\ln \rho_{\text {center }}, \ln T_{\text {center }}, X(\mathrm{H})_{\text {center }}, X(\mathrm{He})_{\text {center }}$.

9. Magnitude of the change in $\log \left(M / M_{\odot}\right)$ due to winds or accretion.

10. Mass accreted so that compressional heating is correct (see Section 5.3).

11. Changes in the logarithm of the total angular momentum.

12. Distance moved in the H-R diagram.

13. Maximum allowed time step under any circumstance.

14. Any user specified time step limit, accomplished by setting max_years_for_time step, in the optional routine extras_check_model.

For convergence studies with respect to the time step it is vital to change the control parameters that are actually setting the time step. Often, this is just varcontrol_target, but in many situations the time step will be set by one of the special time step control parameters.

\section{B.4. Mesh Controls}

Control of the spatial mesh is a key ingredient of a stellar evolution instrument, and requires careful trade-offs. The mesh must respond to gradients in the structure, chemical composition, and energy generation, in order to give an accurate result, but it should not be overly dense since that will unnecessarily increase the cost of the calculation.

Since MESAstar allows for simulations with a fixed inner core mass, $M_{\mathrm{c}}$, the total mass $M$ is $M_{\mathrm{c}}+M_{\mathrm{m}}$ where $M_{\mathrm{m}}$ is the modeled mass. For cell $k$, MESAstar stores the relative cell mass $d q_{k}=d m_{k} / M_{\mathrm{m}}$ where $d m_{k}$ is the mass contained in cell $k$. The relative mass interior to the outer cell face is $q_{k}=1-\sum_{i=1}^{i=k-1} d q_{i}$, and the total mass interior to the outer cell face is $m_{k}=q_{k} * M_{\mathrm{m}}+M_{\mathrm{c}}$. In all cases, $m_{1}=M$ and $q_{1}=1$. We explicitly keep $d q_{k}$ in addition to $q$ and define $q$ in terms of $d q_{k}$ to avoid the need for evaluating $q_{k}-q_{k+1}$ since that can involve the subtraction of almost equal numbers leading to an undesirable loss of precision (Lesaffre et al. 2006). For example, in the outer envelope of a star where the $q_{k}$ approach 1 , the $d q_{k}$ can be $10^{-12}$ or smaller. By storing $d q_{k}$ we have 16 digit precision, whereas in this case, $q_{k}-q_{k+1}$ would only give us four digits at best for the relative cell mass.

MESAstar checks the structure and composition profiles of the model at the beginning of each time step and, if necessary, adjusts the mesh. A single cell can be split into two or more cells and two or more adjacent cells can be merged. In practice, only a small fraction of the cells are changed during a remesh. This minimizes numerical diffusion, aids convergence, and keeps the cost of remeshing relatively small. Remeshing is divided into a planning stage and an adjustment stage. 
The planning stage determines which cells to split or merge based on the magnitude of allowed cell-to-cell changes in a variety of mesh functions. Built-in mesh functions include gradients of the mass, radius, pressure, temperature, adiabatic gradient, angular velocity and mass fractions above some threshold. Users can add others by defining their own other_mesh_functions routine.

Other controls are provided to increase the sensitivity in regions selected by the user. Examples include increasing the spatial resolution in regions with changes in user-specified abundances with respect to pressure, changes in the energy generation rate with respect to pressure for different types of burning (e.g., the pp chains, CNO cycles, triple- $\alpha$, and others), for regions near burning or non-burning convective boundaries, and others.

After the mesh functions are evaluated, the relative magnitude of the changes between adjacent cells are determined. The magnitude of change is multiplied by mesh_delta_coeff to obtain a weighted mesh function. Cells where the weighted changes are "too large" are marked for splitting, and cells where the changes are "too small" are marked for merging. For example, if the weighted changes in all mesh functions from cells $k$ to $k+n$ are less than 1 , the series of cells from $k$ to $k+n$ are marked for merging. If any weighted mesh function changes from cell $k$ to $k+1$ by an amount greater than 1, the larger of cell $k$ and cell $k+1$ is marked for splitting. Finally, if adjacent cells have too large of a relative size difference (as defined by mesh_max_allowed_ratio which defaults to 2.5), the larger cell is marked for splitting and the check for excessive ratios is repeated. This can lead to a cascade of splitting in order to ensure that cells sizes do not have excessive jumps.

The adjustment stage executes the remesh plan by performing the merge and split operations to calculate new values for basic variables. Special care is taken to use physical knowledge whenever possible when setting new values. For example, conservation of mass is accounted for when determining new densities, and species conservation is used when setting new mass fractions. Energy conservation is used when setting the temperature (see Paper I), and conservation of angular momentum plays a role in determining the angular velocity. Cells to be split are constructed by first performing a monotonicitypreserving cubic interpolation (Steffen 1990) in mass to obtain the luminosities and $\ln r$ values at the new cell boundaries. The new densities are then calculated from the new cell masses and volumes. Next, new composition mass fraction vectors are calculated. For cells being merged, the mass averaged abundances are used. For cells being split, neighboring cells are used to form a linear approximation of mass fraction for each species as a function of mass coordinate within the cell. The slopes are adjusted so that the mass fractions sum to one everywhere, and the functions are integrated over the new cell mass to determine the abundances.

\section{B.5. Mass Adjustment}

Mass adjustment for mass loss or accretion is performed at each time step when do_evolve_step calls the routine do_adjust_mass (see Appendix B.1). MESAstar offers several ways to set the rate of mass change $\dot{M}$. A constant mass accretion rate (positive $\dot{M}$ ) or mass loss rate (negative $\dot{M}$ ) can be specified in the input files, a wind can produce a mass loss, the user can set $\dot{M}$ in an other_wind routine or in an other_check_model routine. When do_adjust_mass is called, the time step $d t$ and the rate of mass change $\dot{M}$ are known, and thus the change in mass, $\delta M=\dot{M} \delta t$.

When there is a change in mass, instead of adding or removing cells, the total mass is changed by modifying the modeled mass $M_{\mathrm{m}}$, and cell mass sizes are changed by revisions to $d q_{k}$ which in turn changes cell mass locations $q_{k}$ (see Appendix B.4). The mass structure is divided into an inner region where the $m_{k}$ and $d m_{k}$ are unchanged but the $q_{k}$ and $d q_{k}$ change, an outer region where the $q_{k}$ and $d q_{k}$ are unchanged but the $m_{k}$ and $d m_{k}$ change, and an intermediate blending region where all of these change. The selection of the region boundaries is discussed in detail in Paper I. The implementation of $\epsilon_{\text {grav }}$ in the newly accreted matter is described in Section 5.3.

Once the three regions have been defined, the $d q_{k}$ are updated. In the inner region they are rescaled by $M /(M+\delta M)$. Thus, $d m_{k}, m_{k}$, and $X_{k}$ have the same value before and after a change in mass. This eliminates the possibility of unwanted numerical diffusion causing unphysical mixing in the center region. In the outer region, cells retain the same value of $d q_{k}$ to improve convergence in the high entropy parts of the star (Sugimoto et al. 1981). In the intermediate region, the $d q_{k}$ are uniformly scaled to make $\sum d q_{k}=1$.

The chemical mass fractions of cells in the intermediate and outer regions are then updated by summing the abundances between the new cell mass boundaries. This step is not necessary for the inner region since those cells have not changed mass location. In the case of mass accretion, the composition of the outermost cells whose enclosed mass totals $\delta M$ is set to match the specified accretion abundances. The single cell that is part old material and part newly accreted material is given an appropriately mixed composition.

Finally, to create a somewhat better starting model for the Newton iterations (see Appendix B.2), the $\ln T$ and $\ln \rho$ and $\ln P_{\text {gas }}$ values are revised by monotonic cubic interpolating to the cell center by mass from the values prior to mass adjustment. The $\ln r$ and material speed $v$ are also set by monotonic cubic interpolation to the value at the new outer mass boundary. The angular velocity is set by integrating the angular momentum between the new cell mass boundaries and using the new $\ln r$ values, conserving the total angular momentum to the floating point limit of the arithmetic.

\section{B.6. Evolving the Angular Velocity}

Initialization of rotation in MESAstar begins from a nonrotating model. The angular velocity $\omega$ is added to the set of model variables and initialized to a constant value throughout the model (i.e., solid body rotation). The initial value of $\omega$ can be specified as a surface rotational velocity (in $\mathrm{km} \mathrm{s}^{-1}$ ) or as a fraction of the surface critical rotation rate (see Section 6). During the subsequent evolution, $\omega$ is changed at each time step by remeshing, mass adjustment, radius adjustment (as part of the structure evolution), optional extra angular momentum removal in the outer layers, and the transport of angular momentum optionally with user-defined source terms for external torques.

The angular velocity $\omega$ is defined at cell boundaries. Thus omega (k) is at the outer boundary of cell $\mathrm{k}$, which is the same location as the radius, $r(\mathrm{k})$, the specific moment of inertia, $i \_r o t(k)$, and the specific angular momentum, j_rot (k). The mass associated with omega $(k)$ spans the range from the center by mass of cell $k$ outward to the center by mass of cell $k-1$ and is referred to as dm_bar $(\mathrm{k})$ to distinguish it from the cell mass $\mathrm{dm}(\mathrm{k})$. 
The remeshing operation splits and merges cells but does not change the physical stellar structure (see Appendix B.4). For regions where there has been a change in the mesh, the values of $\omega$ are adjusted to give the same angular momentum as before. More specifically, the angular momentum from the original model is summed over the mass range encompassed by the new dm_bar $(\mathrm{k})$, and omega $(\mathrm{k})$ is adjusted to give the same total for the new model.

During the mass adjustment operation, when mass is added or removed from the model, cells in the outer layers are moved to new mass locations (see Appendix B.5). As part of this process, the angular velocity values are updated to conserve angular momentum using the same scheme as for remeshing: sum the angular momentum in the original model and set omega $(\mathrm{k})$ in the new model to conserve it. Newly added material from accretion is given the current surface angular velocity. In the case of mass loss, this operation removes the amount of angular momentum contained in the lost mass at the start of the time step; it does not deal with possible transport of angular momentum into the lost mass during the time step. That is dealt with by an optional, user-specified removal prior to the angular momentum transport.

MESAstar performs the transport of angular momentum as a separate operation from the evolution of structure and composition. This is done in order to obtain high accuracy in the angular momentum transport by using substeps and quadprecision linear algebra. It does not introduce additional operator splitting errors since $\omega$ is not used in the structure and abundance equations. So we solve for the new structure and composition after any mass change and before the transport of angular momentum. Calculation of the new stellar structure changes the radii but does not change the mass partitioning of the model (see Appendix B.2). Given the new radius $r(\mathrm{k})$, we calculate the new i_rot $(k)$. Then using the unchanged j_rot $(k)$, $\operatorname{omega}(k)$ is set to $j \_r o t(k) / i \_r o t(k)$ to conserve specific angular momentum. Since $d m_{-} b a r(k)$ has not changed, this also conserves total angular momentum.

Next, MESAstar applies an optional, user-specified amount of angular momentum loss in the outer surface layers. This is to account for possible transport of angular momentum during the time step from these outer layers into the mass removed by the mass adjustment operation.

The final operation is the transport of angular momentum within the star, which is treated with a diffusion approximation (Endal \& Sofia 1978; Pinsonneault et al. 1989; Heger et al. 2000)

$$
\begin{aligned}
\left(\frac{\partial \omega}{\partial t}\right)_{m}= & \frac{1}{i}\left(\frac{\partial}{\partial m}\right)_{t}\left[\left(4 \pi r^{2} \rho\right)^{2} i v\left(\frac{\partial \omega}{\partial m}\right)_{t}\right] \\
& -\frac{2 \omega}{r}\left(\frac{\partial r}{\partial t}\right)_{m}\left(\frac{1}{2} \frac{d \ln i}{d \ln r}\right)
\end{aligned}
$$

where $i$ is the specific moment of inertia of a shell at mass coordinate $m$, and $v$ is the turbulent viscosity determined as the sum of the diffusion coefficients for convection, double diffusion, overshooting and rotationally induced instabilities (see Section 6). The diffusive transport is carefully implemented to accurately conserve angular momentum. The angular momentum associated with location $k$ is dm_bar $(\mathrm{k}) * i_{\text {_rot }}(\mathrm{k}) * \operatorname{omega}(\mathrm{k})$. The change in angular momentum for $k$ is determined by the flux in angular momentum from $k-1$ to $k$ and from $k+1$ to $k$. The flux from $k-1$ to $k$ is set by $v(k-1)$ and the difference between omega $(\mathrm{k})$ and omega $(\mathrm{k}-1)$. The flux from $k+1$ to $k$ is found similarly using $v(k)$ and the difference between omega $(\mathrm{k})$ and omega $(k+1)$. Source terms for location $k$ are applied by usersupplied values for extra_jdot (k) or extra_omegadot $(k)$. The finite difference equation for the effects of the transport and source terms is solved over the stellar time step with an implicit time integration that uses multiple smaller time steps. The sizes of these substeps are determined by the timescale set by the diffusion coefficients and the differences in $\omega$. It is not unusual to use 10 or more substeps to evolve omega ( $\mathrm{k}$ ) over the stellar time step. Each implicit substep is solved using a quad-precision tridiagonal matrix routine. The conservation of total angular momentum is monitored and the stellar time step is rejected if there is any deviation from conservation by more than a user-specified factor. In practice, we find the total angular momentum is conserved over the stellar time step to within a few digits of the floating point limit of the arithmetic.

\section{B.7. Free Parameters}

Stellar evolution calculations involve the choice of a number of free parameters. The values of these parameters are not determined by first principles, and in the literature one can find a range of possibilities. In some cases the parameters can be constrained by matching a restricted set of observations; in other cases they represent common choices. Users need to be aware that their results will depend on these values, and that in some cases the sensitivity can be large. Below we illustrate this by discussing some of the main parameters involved in the mixing of stellar interiors.

\section{B.7.1. Convection}

In the literature the value of the mixing length parameter $\alpha_{\text {MLT }}$ (see, e.g., Paper I for a definition) is usually found to vary within the range $1.0 \lesssim \alpha_{\mathrm{MLT}} \lesssim 2.0$. Efforts are ongoing to eliminate this free parameter (e.g., Arnett et al. 2010).

\section{B.7.2. Overshooting}

In the literature the adopted value for the convective core overshooting parameter is in the range $0.1 \lesssim f_{\text {ov }} \lesssim 0.6$, in units of the pressure scale height $\lambda_{P}$, when the overshoot zone is considered to be fully mixed (Maeder \& Meynet 1987; Dupret et al. 2004; Straka et al. 2005; Claret 2007; Briquet et al. 2007). When overshoot mixing is treated as an exponential decay process the free parameter should be smaller, $f_{\text {ov }} \sim 0.016$, (see the discussion by Herwig 2000). MESA has the ability to treat overshoot mixing zones as either fully mixed or in the exponential decay formalism.

It has been suggested that the overshooting parameter is a function of both mass and metal abundance, in that it should transition smoothly from zero to a maximum value over a small range of stellar mass where a convective core is present on the MS (Woo \& Demarque 2001; VandenBerg et al. 2006), but see also Claret (2007). A dependency on the evolutionary stage seems also likely (Herwig et al. 1997; Meakin \& Arnett 2007; Tian et al. 2009). See Figure 13-15 in Section 4 for an example of the sensitivity of the calculations to changes in the $f_{\text {ov }}$ parameter.

\section{B.7.3. Semiconvection}

Semiconvection, as implemented in MESA, requires a choice of the free parameter $\alpha_{\mathrm{sc}}$ (see Section 4.1). In the literature this spans the range $0.001 \lesssim \alpha_{\text {sc }} \lesssim 1.0$ (Langer 1991; Yoon et al. 2006). See Figure 13- 15 in Section 4 for an example of the 
sensitivity of the calculations to changes in the $\alpha_{\mathrm{sc}}$ parameter. Research is ongoing to eliminate this free parameter (Wood et al. 2013; Spruit 2013).

\section{B.7.4. Thermohaline Mixing}

The implemented formulation for thermohaline mixing requires the adoption of the free parameter $\alpha_{\text {th }}$ (see Section 4.2). In the literature this parameter can be usually found within the range $1 \lesssim \alpha_{\text {th }} \lesssim 667$ (Kippenhahn et al. 1980; Charbonnel \& Zahn 2007; Cantiello \& Langer 2010; Stancliffe 2010; Wachlin et al. 2011). Research is ongoing to eliminate this free parameter (Traxler et al. 2011; Brown et al. 2013).

\section{B.8. Nuclear Reactions}

A reaction network is defined by a set of isotopes and a set of reactions; these sets are specified in a reaction network definition file. MESA comes with many predefined reaction networks in data/net_data/nets and can also incorporate user-defined networks. To use a custom network, a user creates a reaction network definition file containing the command add_isos_and_reactions(isos_list), which will automatically add all reactions linking the isotopes in isos_list. The sequence of isotopes in isos_list may be specified by the name of the isotope: for example, add_isos_and_reactions(he4) adds ${ }^{4} \mathrm{He}$. Alternatively, one can specify the name of element followed by the desired minimum and maximum nucleon number. For example, the command add_isos_and_reactions (o 16 18) adds ${ }^{16} \mathrm{O}$, ${ }^{17} \mathrm{O}$, and ${ }^{18} \mathrm{O}$. Note that because many of the predefined networks may use effective rates - that is, using one reaction rate to represent a reaction sequence or group of reaction sequences-it is not recommended that the user extend one of the pre-existing networks with this command.

MESA creates and stores reaction rate tables for each reaction whose entries are derived from evaluating standard analytic fitting formulae (see Appendix A.4), but these reaction rates may be replaced with user-specified values. To change a rate for a given reaction,

1. create a file with two columns: the temperature in units of $10^{8} \mathrm{~K}$ and the rate $N_{\mathrm{A}}\langle\sigma v\rangle$ in units of $\mathrm{cm}^{3} \mathrm{~g}^{-1} \mathrm{~s}^{-1}$;

2. list the file name in a local file rate_list.txt along with its "handle" for the reaction rate in question (see discussion below); and

3. set the parameter rate_tables_dir in the namelist star_job to the name of the directory in which rate_list.txt is located; by default this is data/rates/ rate_tables.

The handle for a reaction is derived from the input and output channel isotopes according to a few rules. Capture reactions, such as $x(p, \gamma) y$, have handles of the form $r_{-} \mathrm{x}_{-} \mathrm{pg} \mathrm{g}_{-} \mathrm{y}$ and exchange reactions, such as $x(\alpha, p) y$, have handles of the form $r_{-} x_{-}$ap_y. Other arbitrary reactions may be added by listing them in a form $r_{-}$inputs_to_outputs where inputs and outputs are isotopes separated by "_." If the same isotope appears two or more times, the isotope name may be repeated. For example, the triple- $\alpha$ reaction is specified as r_he4_he4_he4_to_c12. Isotopes are ordered by increasing $Z$ and $N$, e.g., r_h3_be7_to_neut_h1_he4_he4. To see a list of reactions used, the parameter show_net_reactions_info in namelist star_job should be set to .true..

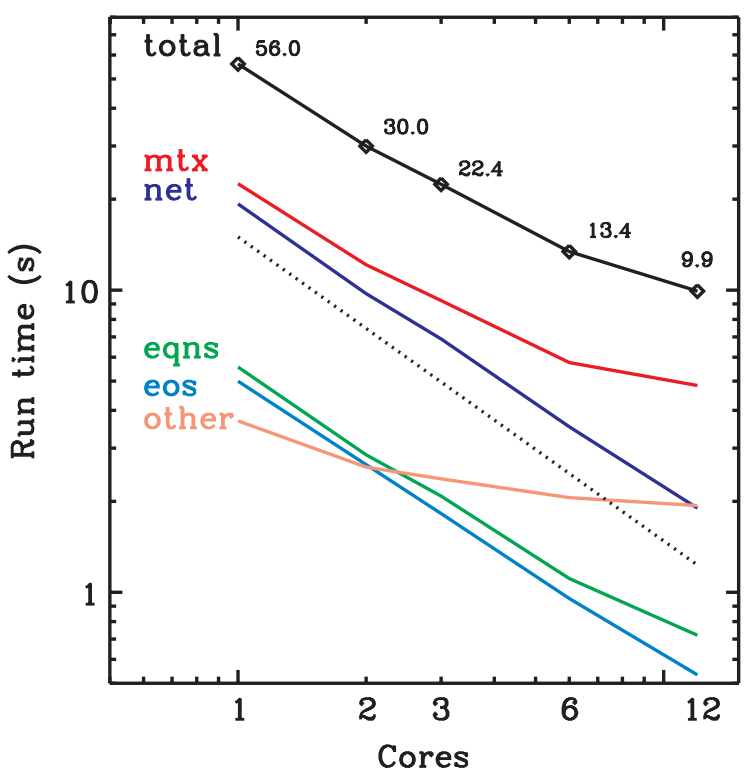

Figure 48. Scaling behavior of various components of MESAstar using 1, 2, 3,6 , and 12 cores. The top curve shows the total run time, and the lower solid curves show the run times for the components of the total. The dotted line shows the ideal scaling relation.

(A color version of this figure is available in the online journal.)

\section{B.9. Multicore Performance}

MESA implements shared memory multiprocessing via OpenMP. ${ }^{11}$ Paper I explored the runtime scaling of MESAstar which at that time used a banded matrix linear algebra solver that did not benefit from multiple cores. A large part of the performance improvement in MESAstar since Paper I comes from converting to a parallel block tridiagonal linear algebra solver derived from BCYCLIC (Hirshman et al. 2010). This improved solver is particularly important since linear algebra is typically the largest part of the runtime in MESAstar. In addition, the new algorithm has the desirable property of producing numerically identical results independent of the number of cores, an attribute that is not generally true of parallel matrix solvers.

Our test case is a $1.5 M_{\odot}$ model with $Z=0.02$ that is evolved from the ZAMS until the central $\mathrm{H}$ mass fraction falls to 0.35 . This model includes 25 isotopes and 4 structure variables per cell with a variable number of zones typically exceeding 1700 . The test takes $\sim 55$ time steps to cover $\sim 1.4$ Gyr and uses the default amount of I/O.

Figure 48 shows the scaling behavior of some key components of MESAstar under GFORTRAN 4.7.2. on a 12 core 2010 Apple MacPro. The dotted line shows the ideal scaling relation where doubling the number of cores cuts the run time in half. The linear algebra, labeled "mtx," dominates the total run time as the number of cores increases. For example, in the case of 12 cores it accounts for about half the total and is 2.5 times larger than "net," the evaluation of the nuclear reaction network. The net evaluations closely approach the ideal scaling behavior because they can be done in parallel, each cell independent of the others, with one core working on one cell at a time. The equation of state component, labeled "eos," also closely approaches the ideal scaling law while consuming less than a third of the run time for the net. The component labeled "eqns," which includes the evaluation of the structure equations and the creation of the block tridiagonal matrix, also is close to the ideal

\footnotetext{
11 http://www.openmp.org
} 
scaling law and costs about the same as the eos. The "other" component is everything else. It is dominated by processes that currently are not efficient to parallelize because of the relatively large overhead for OpenMP operations. Consequently it remains at roughly a constant run time independent of the number of cores. When a significantly larger number of cores per processor becomes available, the larger operations in this category will have to be reworked or they will dominate the total run time.

The run time also depends on the hardware, the quality of the compiled code, and the efficiency of the OpenMP implementation. For example, we ran the test case under GFORTRAN 4.7.2 on a 40-core server. While we obtained a speedup of 5.6 in going from 1 to 12 cores for the 12 core machine (see Figure 48), we find a speedup of only 4.8 on the 40-core server in going from 1 to 12 cores. Moreover, the speedup per core dropped steeply beyond 8-12 cores on the 40-core server, confirming the expectation that much work will be required to make full use of machines with many cores.

\section{B.10. Visualization}

MESAstar provides alphanumeric output at user-specified regular intervals. In addition, the routines in module star/public/pgstar.f provide an option for concurrent graphical output with the PGPLOT ${ }^{12}$ library to create on-screen plots that can be saved for post-processing into animations of an evolutionary sequence. A variety of options are provided and are all configurable through the PGstar inlist. For example, a PGstar X11 window can simultaneously hold an H-R diagram, a $T_{\mathrm{c}}-\rho_{\mathrm{c}}$ diagram, and interior profiles of physical variables, such as nuclear energy generation and composition. The PGstar inlist is read at each time step, so the display options can be changed without have to stop MESAstar.

Since Paper I, a number of MESAstar users have developed and released toolkits ${ }^{13}$ to visualize the alphanumeric output with common graphical packages including: Mathematica scripts (contributed by Richard O'Shaughnessy) and the intuitive and efficient graphical user interface MESAFace (Giannotti et al. 2012); MatLab utilities (contributed by Dave Spiegel and Gongjie Li); IDL functions (contributed by Rich Townsend); Python scripts (contributions from Falk Herwig and the NuGrid collaboration, David Kaplan, Alfred Gautschy, William Wolf); and Tioga scripts (contributed by Christopher Mankovich and Bill Paxton).

\section{B.11. Operating System and Compiler Considerations}

We next consider the implications of running MESA compiled with different compilers and on different operating systems. The operating systems examined are Linux (Gentoo 2.1; kernel 3.6.11) and Apple OS X (10.7.5), both 64-bit; the compilers are GNU gfortran 4.9.0 on Linux and OS X and Intel ifort 13.1.0 on Linux. We used OpenMP in all cases. For optimization we used -02 with gfortran and -01 with ifort. The models described here were computed with MESA revision 4942. The comparison case is the example_solar_model from the test_suite. It evolves a calibrated solar model from the PMS to the solar age, 4.57 Gyr.

In Figure 49 we compare the sound speed profiles of these solar models in the same way that standard solar models are compared with the solar sound speed profile in Section 3.1, Figure 7 . The reference was chosen to be the Linux/gfortran

\footnotetext{
12 http://www.astro.caltech.edu/ tjp/pgplot/.

13 See http://mesastar.org/tools-utilities.
}

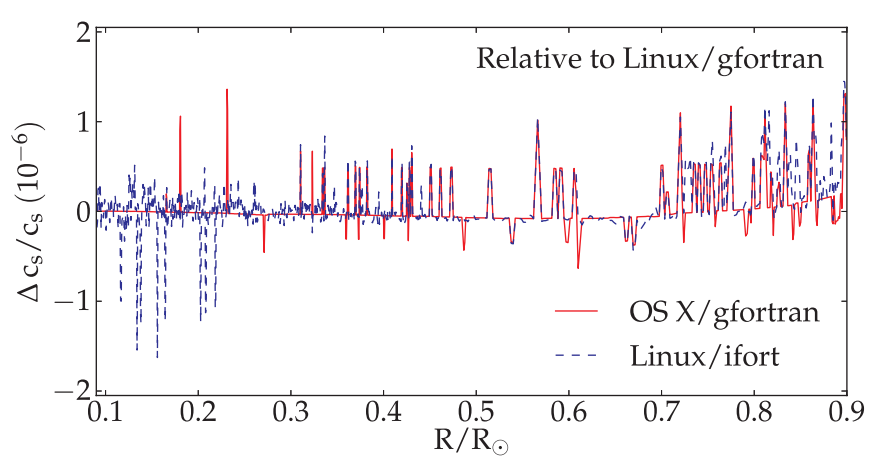

Figure 49. Relative difference in the solar sound speed profile from three models run using the same inlist but on different operating systems and/or compilers. (A color version of this figure is available in the online journal.)

model; the other two models were compared with it by interpolating their sound speed profiles onto the radial grid of the reference model and then taking the relative sound speed difference with respect to that reference model. Figure 49 indicates that models computed on different platforms are consistent with one another at the level of the numerical tolerances with which the equations are solved. The differences shown here are about one part per million or less, whereas the differences between standard solar models and the helioseismic data are in the parts per thousand (Figure 7) - a factor of 1000 difference. While this result is reassuring, it is worthwhile to close this discussion with the comment that the consistency found in a low-mass, nonrotating model evolved about half way through the MS phase will not be representative of other cases dealing with different physics, stellar masses, and evolutionary phases. Projects using MESA with heterogeneous architectures should perform their own consistency checks.

\section{APPENDIX C}

\section{THE MESA SOFTWARE DEVELOPMENT KIT}

MESA is provided as source code, allowing users access to all of the implementation details. Installation necessarily involves building the code from source, which is a non-trivial task. A successful build requires cooperation between the operating system, compiler, libraries, and utilities.

To address this issue we have created the MESA SDK, which packages everything necessary to establish a unified and maintained build environment. ${ }^{14}$ The principal components of the SDK are summarized in Table 6; all of these are distributed under an open-source license (detailed in the table), permitting their redistribution without financial or copyright encumbrances. Perhaps the most important component is the GFORTRAN compiler, part of the GNU Compiler Collection. GFORTRAN implements almost all of the Fortran 2003 (F2003) standard, and benefits from a high level of community support.

The SDK is available for Intel x86 and x86-64 CPU architectures running the Linux and Mac OS X operating systems (these platforms comprise most of the MESA user base). Installation of the kit is straightforward, requiring a tar archive to be unpacked (Linux) or an application folder to be copied (OS X), followed by the initialization of a few environment variables. By default, MESA is configured to compile "out-of-the-box" with the SDK. MESA can also be compiled without the SDK, using any alternate compiler which supports the F2003 standard. In this respect,

14 Available from http://www.astro.wisc.edu/ townsend/static.php?
ref $=$ mesasdk 
Table 6

Principal Components of the MESA Software Development Kit

\begin{tabular}{lccl}
\hline \hline Name & Purpose & Version & \multicolumn{1}{c}{ License $^{\mathrm{a}}$} \\
\hline GFORTRAN & Compiler & 4.7 .2 & Open source (GPL ver. 2) \\
BLAS & Matrix algebra & $2011-04-19$ & Open source (other) \\
LAPACK & Matrix algebra & 3.4 .2 & Open source (other) \\
HDF5 & File storage & 1.8 .9 & Open source (other) \\
NDIFF & Numerical comparison & 2.00 & Open source (GPL ver. 2) \\
PGPLOT & Plotting & 5.2 .2 & Open source (non-commercial) \\
SE & File storage & 1.2 .1 & Open source (other) \\
\hline
\end{tabular}

Note. " "GPL" denotes the GNU General Public License (with the version in parentheses); "non-commercial" denotes an open-source license with restrictions on commercial distribution; and "other" denotes to a variety of open-source licenses which permit largely unrestricted distribution.

GFORTRAN should not be viewed as the MESA compiler (nor the full SDK as the MESA build environment). MESA will adhere to Fortran standards rather than rely on vendor-specific extensions.

Uptake of the SDK has been very rapid: at the time of writing, we estimate over $90 \%$ of the MESA community (over 500 users) are using the SDK. This growth has been matched by a significant decline in the number of installation support requests, and a corresponding reduction in the time taken to resolve these requests. With these maintenance overheads curbed, the MESA developers are able to devote more of their time to refining and extending the code.

\section{REFERENCES}

Alibert, Y., Mordasini, C., \& Benz, W. 2011, A\&A, 526, A63

Allard, F., Hauschildt, P. H., Alexander, D. R., Tamanai, A., \& Schweitzer, A. 2001, ApJ, 556, 357

Althaus, L. G., Serenelli, A. M., Córsico, A. H., \& Montgomery, M. H. 2003, A\&A, 404, 593

Angulo, C., Arnould, M., Rayet, M., et al. 1999, NuPhA, 656, 3

Arcoragi, J.-P., \& Fontaine, G. 1980, ApJ, 242, 1208

Arnett, D., Meakin, C., \& Young, P. A. 2010, ApJ, 710, 1619

Baglin, A., Auvergne, M., Barge, P., et al. 2009, in IAU Symp. 253, Transiting Planets, ed. F. Pont, D. Sasselov, \& M. J. Holman (Cambridge: Cambridge Univ. Press), 71

Bahcall, J. N., Basu, S., \& Pinsonneault, M. H. 1998, PhLB, 433, 1

Balbus, S. A., Latter, H., \& Weiss, N. 2012, MNRAS, 420, 2457

Bass, G., Orosz, J. A., Welsh, W. F., et al. 2012, ApJ, 761, 157

Beck, P. G., Montalban, J., Kallinger, T., et al. 2012, Natur, 481, 55

Bergeron, P., Wesemael, F., Lamontagne, R., et al. 1995, ApJ, 449, 258

Bildsten, L., Paxton, B., Moore, K., \& Macias, P. J. 2012, ApJL, 744, L6

Bisnovatyi-Kogan, G. S., \& Dorodnitsyn, A. V. 1999, A\&A, 344, 647

Bloecker, T. 1995, A\&A, 297, 727

Böhm, K. H., \& Cassinelli, J. 1971, A\&A, 12, 21

Böhm-Vitense, E. 1958, ZA, 46, 108

Bonanno, A., Küker, M., \& Paternò, L. 2007, A\&A, 462, 1031

Borucki, W., Koch, D., Batalha, N., et al. 2009, in IAU Symp. 253, Transiting Planets, ed. F. Pont, D. Sasselov, \& M. J. Holman (Cambridge: Cambridge Univ. Press), 289

Boss, A. P. 2011, ApJ, 731, 74

Braithwaite, J. 2006, A\&A, 449, 451

Braithwaite, J., \& Spruit, H. C. 2004, Natur, 431, 819

Brandenburg, A., \& Subramanian, K. 2005, PhR, 417, 1

Brassard, P., Fontaine, G., Wesemael, F., Kawaler, S. D., \& Tassoul, M. 1991, ApJ, 367, 601

Briquet, M., Morel, T., Thoul, A., et al. 2007, MNRAS, 381, 1482

Brott, I., de Mink, S. E., Cantiello, M., et al. 2011, A\&A, 530, A115

Brown, J. M., Garaud, P., \& Stellmach, S. 2013, ApJ, 768, 34

Brown, T. M., Christensen-Dalsgaard, J., Dziembowski, W. A., et al. 1989, ApJ, 343,526

Brown, T. M., Gilliland, R. L., Noyes, R. W., \& Ramsey, L. W. 1991, ApJ, 368, 599

Burkart, J., Quataert, E., Arras, P., \& Weinberg, N. N. 2012, MNRAS, 421, 983

Burkart, J., Quataert, E., Arras, P., \& Weinberg, N. N. 2013, MNRAS, 1449

Calder, A. C., Townsley, D. M., Seitenzahl, I. R., et al. 2007, ApJ, 656, 313
Cantiello, M., \& Braithwaite, J. 2011, A\&A, 534, A140

Cantiello, M., \& Langer, N. 2010, A\&A, 521, A9

Cantiello, M., Langer, N., Brott, I., et al. 2009, A\&A, 499, 279

Carlberg, J. K., Cunha, K., Smith, V. V., \& Majewski, S. R. 2012, ApJ, 757, 109

Carter, J. A., Fabrycky, D. C., Ragozzine, D., et al. 2011, Sci, 331, 562

Cassisi, S., Potekhin, A. Y., Pietrinferni, A., Catelan, M., \& Salaris, M. 2007, ApJ, 661, 1094

Chang, P., \& Hui, L. 2011, ApJ, 732, 25

Charbonnel, C., \& Talon, S. 2005, Sci, 309, 2189

Charbonnel, C., \& Zahn, J.-P. 2007, A\&A, 467, L15

Chatzopoulos, E., Robinson, E. L., \& Wheeler, J. C. 2012, ApJ, 755, 95

Chatzopoulos, E., \& Wheeler, J. C. 2012, ApJ, 748, 42

Christensen-Dalsgaard, J. 2008a, Ap\&SS, 316, 113

Christensen-Dalsgaard, J. 2008b, Ap\&SS, 316, 13

Christensen-Dalsgaard, J., Dappen, W., Ajukov, S. V., et al. 1996, Sci, 272, 1286

Christensen-Dalsgaard, J., \& Thompson, M. J. 1997, MNRAS, 284, 527

Claret, A. 2007, A\&A, 475, 1019

Cox, J. P., \& Giuli, R. T. 1968, Principles of Stellar Structure (New York: Gordon and Breach)

Cyburt, R. H., Amthor, A. M., Ferguson, R., et al. 2010, ApJS, 189, 240

Davis, A.-C., Lim, E. A., Sakstein, J., \& Shaw, D. J. 2012, PhRvD, 85, 123006

D'Cruz, N. L., Dorman, B., Rood, R. T., \& O'Connell, R. W. 1996, ApJ, 466, 359

de Bruijne, J. H. J. 2012, Ap\&SS, 341, 31

Deheuvels, S., Bruntt, H., Michel, E., et al. 2010, A\&A, 515, 87

Deheuvels, S., \& Michel, E. 2011, A\&A, 535, A91

de Jager, C., Nieuwenhuijzen, H., \& van der Hucht, K. A. 1988, A\&AS, 72, 259

Denissenkov, P. A. 2010, ApJ, 723, 563

Denissenkov, P. A. 2012, ApJL, 753, L3

Denissenkov, P. A., Herwig, F., Bildsten, L., \& Paxton, B. 2013, ApJ, 762, 8

Denissenkov, P. A., \& Pinsonneault, M. 2007, ApJ, 655, 1157

Denissenkov, P. A., \& Pinsonneault, M. 2008, ApJ, 684, 626

Denissenkov, P. A., Pinsonneault, M., Terndrup, D. M., \& Newsham, G. 2010, ApJ, 716, 1269

Dotter, A., Chaboyer, B., Jevremović, D., et al. 2008, ApJS, 178, 89

Dupret, M.-A., Thoul, A., Scuflaire, R., et al. 2004, A\&A, 415, 251

Eggenberger, P., Meynet, G., Maeder, A., et al. 2008, Ap\&SS, 316, 43

Eggenberger, P., Montalbán, J., \& Miglio, A. 2012, A\&A, 544, L4

Ekström, S., Georgy, C., Eggenberger, P., et al. 2012, A\&A, 537, A146

Endal, A. S., \& Sofia, S. 1976, ApJ, 210, 184

Endal, A. S., \& Sofia, S. 1978, ApJ, 220, 279

Evans, C. J., Taylor, W. D., Hénault-Brunet, V., et al. 2011, A\&A, 530, A108

Ferguson, J. W., Alexander, D. R., Allard, F., et al. 2005, ApJ, 623, 585

Fischer, D. A., \& Valenti, J. 2005, ApJ, 622, 1102

Fortney, J. J., Saumon, D., Marley, M. S., Lodders, K., \& Freedman, R. S. 2006, ApJ, 642, 495

Freedman, R. S., Marley, M. S., \& Lodders, K. 2008, ApJS, 174, 504

Friend, D. B., \& Abbott, D. C. 1986, ApJ, 311, 701

Frommhold, L., Abel, M., Wang, F., et al. 2010, MolPh, 108, 2265

Fuller, J., \& Lai, D. 2012, ApJL, 756, L17

Fuller, J., \& Lai, D. 2013, MNRAS, 430, 274

Georgy, C., Ekström, S., Meynet, G., et al. 2012, A\&A, 542, A29

Georgy, C., Meynet, G., \& Maeder, A. 2011, A\&A, 527, A52

Giannotti, M., Wise, M., \& Mohammed, A. 2013, CoPhC, 184, 1333

Glebbeek, E., Gaburov, E., de Mink, S. E., Pols, O. R., \& Portegies Zwart, S. F. 2009, A\&A, 497, 255

Gough, D. O. 1986, in Hydrodynamic and Magnetodynamic Problems in the Sun and Stars, ed. Y. Osaki (Tokyo: Univ. Tokyo Press), 117 
Grevesse, N., \& Noels, A. 1993, in Origin and Evolution of the Elements, ed. N. Prantzos, E. Vangioni-Flam, \& M. Casse (Cambridge: Cambridge Univ. Press), 15

Grevesse, N., \& Sauval, A. J. 1998, SSRv, 85, 161

Guillot, T. 2005, AREPS, 33, 493

Guillot, T. 2010, A\&A, 520, A27

Guillot, T., \& Morel, P. 1995, A\&AS, 109, 109

Hairer, E., \& Wanner, G. 1996, Solving Ordinary Differential Equations. II. Stiff and Differential-Algebraic Problems (2nd ed.; Berlin: Springer)

Heger, A., Jeannin, L., Langer, N., \& Baraffe, I. 1997, A\&A, 327, 224

Heger, A., \& Langer, N. 1998, A\&A, 334, 210

Heger, A., \& Langer, N. 2000, ApJ, 544, 1016

Heger, A., Langer, N., \& Woosley, S. E. 2000, ApJ, 528, 368

Heger, A., Woosley, S. E., \& Spruit, H. C. 2005, ApJ, 626, 350

Henyey, L., Vardya, M. S., \& Bodenheimer, P. 1965, ApJ, 142, 841

Herwig, F. 2000, A\&A, 360, 952

Herwig, F. 2004, ApJS, 155, 651

Herwig, F., Bloecker, T., Schoenberner, D., \& El Eid, M. 1997, A\&A, 324, L81

Hirschi, R., Meynet, G., \& Maeder, A. 2004, A\&A, 425, 649

Hirshman, S. P., Perumalla, K. S., Lynch, V. E., \& Sanchez, R. 2010, JCoPh, 229,6392

Hooke, R., \& Jeeves, T. A. 1961, J. ACM, 8, 212

Huang, X., \& Cumming, A. 2012, ApJ, 757, 47

Hubickyj, O., Bodenheimer, P., \& Lissauer, J. J. 2005, Icar, 179, 415

Humphreys, R. M., \& Davidson, K. 1994, PASP, 106, 1025

Hunter, I., Dufton, P. L., Smartt, S. J., et al. 2007, A\&A, 466, 277

Iben, I., Jr. 1991, ApJS, 76, 55

Iglesias, C. A., \& Rogers, F. J. 1993, ApJ, 412, 752

Iglesias, C. A., \& Rogers, F. J. 1996, ApJ, 464, 943

Iglesias, C. A., Rogers, F. J., \& Wilson, B. G. 1992, ApJ, 397, 717

Imbriani, G., Costantini, H., Formicola, A., et al. 2004, A\&A, 420, 625

Irwin, J. M., Quinn, S. N., Berta, Z. K., et al. 2011, ApJ, 742, 123

Ivezic, Z., Tyson, J. A., Acosta, E., et al. 2008, arXiv:0805.2366

Joss, P. C., Salpeter, E. E., \& Ostriker, J. P. 1973, ApJ, 181, 429

Kaiser, N., Burgett, W., Chambers, K., et al. 2010, Proc. SPIE, 7733, 77330E

Kawaler, S. D. 1988, ApJ, 333, 236

Kippenhahn, R., Ruschenplatt, G., \& Thomas, H.-C. 1980, A\&A, 91, 175

Kippenhahn, R., \& Thomas, H.-C. 1970, in IAU Colloq. 4, Stellar Rotation, ed. A. Slettebak (London: Gordon and Breach Science Publishers), 20

Kjeldsen, H., \& Bedding, T. R. 1995, A\&A, 293, 87

Kjeldsen, H., Bedding, T. R., \& Christensen-Dalsgaard, J. 2008, ApJL, 683, L175

Koch, D. G., Borucki, W. J., Basri, G., et al. 2010, ApJL, 713, L79

Krishnamurti, R. 2003, JFM, 483, 287

Küker, M., Rüdiger, G., \& Kitchatinov, L. L. 2011, A\&A, 530, A48

Kunz, R., Fey, M., Jaeger, M., et al. 2002, ApJ, 567, 643

Langer, N. 1991, A\&A, 252, 669

Langer, N. 1997, in ASP Conf. Ser. 120, Luminous Blue Variables: Massive Stars in Transition, ed. A. Nota \& H. Lamers (San Francisco, CA: ASP), 83 Langer, N. 1998, A\&A, 329, 551

Langer, N. 2012, ARA\&A, 50, 107

Langer, N., Fricke, K. J., \& Sugimoto, D. 1983, A\&A, 126, 207

Langer, N., Heger, A., Wellstein, S., \& Herwig, F. 1999, A\&A, 346, L37

Laughlin, G., Bodenheimer, P., \& Adams, F. C. 2004, ApJL, 612, L73

Law, N. M., Kulkarni, S. R., Dekany, R. G., et al. 2009, PASP, 121, 1395

Lesaffre, P., Han, Z., Tout, C. A., Podsiadlowski, P., \& Martin, R. G. 2006, MNRAS, 368, 187

Li, Y., \& Gong, Z. G. 1994, A\&A, 289, 449

Liu, C., Bailer-Jones, C. A. L., Sordo, R., et al. 2012, MNRAS, 426, 2463

Lloyd, J. P. 2011, ApJL, 739, L49

Lodders, K. 2003, ApJ, 591, 1220

MacDonald, J., \& Mullan, D. J. 2012, MNRAS, 421, 3084

Maeder, A. 1987a, A\&A, 173, 247

Maeder, A. 1987b, A\&A, 178, 159

Maeder, A. 2009, Physics, Formation and Evolution of Rotating Stars (Berlin: Springer)

Maeder, A., \& Meynet, G. 1987, A\&A, 182, 243

Maeder, A., \& Meynet, G. 2000a, A\&A, 361, 159

Maeder, A., \& Meynet, G. 2000b, ARA\&A, 38, 143

Maeder, A., \& Meynet, G. 2003, A\&A, 411, 543

Maeder, A., \& Meynet, G. 2004, A\&A, 422, 225

Maeder, A., \& Zahn, J.-P. 1998, A\&A, 334, 1000

Marley, M. S., Fortney, J. J., Hubickyj, O., Bodenheimer, P., \& Lissauer, J. J. 2007, ApJ, 655, 541

Mathur, S., Metcalfe, T. S., Woitaszek, M., et al. 2012, ApJ, 749, 152

Meakin, C. A., \& Arnett, D. 2007, ApJ, 667, 448

Medin, Z., \& Cumming, A. 2010, PhRvE, 81, 036107
Metcalfe, T. S., Chaplin, W. J., Appourchaux, T., et al. 2012, ApJL, 748, L10

Meynet, G., Eggenberger, P., \& Maeder, A. 2011, A\&A, 525, L11

Meynet, G., \& Maeder, A. 1997, A\&A, 321, 465

Meynet, G., \& Maeder, A. 2000, A\&A, 361, 101

Mihalas, D. 1978, Stellar Atmospheres (2nd ed.; San Francisco: Freeman)

Mohr, P. J., Taylor, B. N., \& Newell, D. B. 2008, RvMP, 80, 633

Monteiro, M. J. P. F. G. (ed.) 2009, in Evolution and Seismic Tools for Stellar Astrophysics (Astrophysics and Space Science, Vol. 316; Berlin: Springer), 261

Moravveji, E., Moya, A., \& Guinan, E. F. 2012, ApJ, 749, 74

Mosser, B., Goupil, M. J., Belkacem, K., et al. 2012, A\&A, 548, A10

Mugrauer, M., \& Neuhäuser, R. 2009, A\&A, 494, 373

Nomoto, K. 1982, ApJ, 253, 798

Nomoto, K., \& Sugimoto, D. 1977, PASJ, 29, 765

Nugis, T., \& Lamers, H. J. G. L. M. 2000, A\&A, 360, 227

Owen, J. E., \& Wu, Y. 2013, arXiv:1303.3899

Owocki, S. P., Gayley, K. G., \& Shaviv, N. J. 2004, ApJ, 616, 525

Paczyński, B. 1969, AcA, 19, 1

Paczyński, B. 1970, AcA, 20, 47

Pamyatnykh, A. A. 1999, AcA, 49, 119

Passy, J.-C., Mac Low, M.-M., \& De Marco, O. 2012, ApJL, 759, L30

Patience, J., White, R. J., Ghez, A. M., et al. 2002, ApJ, 581, 654

Paxton, B., Bildsten, L., Dotter, A., et al. 2010, ApJS, 192, 3

Petrovic, J., Langer, N., Yoon, S.-C., \& Heger, A. 2005, A\&A, 435, 247

Pinsonneault, M. H., Kawaler, S. D., Sofia, S., \& Demarque, P. 1989, ApJ, 338,424

Pollack, J. B., Hubickyj, O., Bodenheimer, P., et al. 1996, Icar, 124, 62

Potekhin, A. Y., \& Chabrier, G. 2010, CoPP, 50, 82

Potter, A. T., Tout, C. A., \& Brott, I. 2012a, MNRAS, 423, 1221

Potter, A. T., Tout, C. A., \& Eldridge, J. J. 2012b, MNRAS, 419, 748

Powell, M. 2009, The BOBYQA Algorithm for Bound Optimization without Derivatives (Cambridge: Dept. of Applied Mathematics and Theoretical Physics, Univ. of Cambridge)

Rauscher, T., \& Thielemann, F. 2000, ADNDT, 75, 1

Reimers, D. 1975, in Problems in Stellar Atmospheres and Envelopes, ed. B. Baschek, W. H. Kegel, \& G. Traving (New York: Springer), 229

Roell, T., Neuhäuser, R., Seifahrt, A., \& Mugrauer, M. 2012, A\&A, 542, A92

Rogers, F. J., \& Nayfonov, A. 2002, ApJ, 576, 1064

Rohrmann, R. D. 2001, MNRAS, 323, 699

Rohrmann, R. D., Althaus, L. G., García-Berro, E., Córsico, A. H., \& Miller Bertolami, M. M. 2012, A\&A, 546, 119

Roxburgh, I. W., \& Vorontsov, S. V. 2003, A\&A, 411, 215

Ruediger, G., von Rekowski, B., Donahue, R. A., \& Baliunas, S. L. 1998, ApJ, 494, 691

Sato, B., Fischer, D. A., Henry, G. W., et al. 2005, ApJ, 633, 465

Saumon, D., Chabrier, G., \& van Horn, H. M. 1995, ApJS, 99, 713

Schatzman, E. 1962, AnAp, 25, 18

Schneider, A. S., Hughto, J., Horowitz, C. J., \& Berry, D. K. 2012, PhRvE, 85, 066405

Schou, J., Antia, H. M., Basu, S., et al. 1998, ApJ, 505, 390

Seager, S., \& Deming, D. 2010, ARA\&A, 48, 631

Silva Aguirre, V., Basu, S., Brandão, I. M., et al. 2013, ApJ, 769, 141

Söderlind, G., \& Wang, L. 2006, JCoAM, 185, 225

Spruit, H. C. 2002, A\&A, 381, 923

Spruit, H. C. 2013, A\&A, 552, A76

Stancliffe, R. J. 2010, MNRAS, 403, 505

Steffen, M. 1990, A\&A, 239, 443

Stello, D., Chaplin, W. J., Bruntt, H., et al. 2009, ApJ, 700, 1589

Straka, C. W., Demarque, P., \& Guenther, D. B. 2005, ApJ, 629, 1075

Straniero, O., Domínguez, I., Imbriani, G., \& Piersanti, L. 2003, ApJ, 583, 878

Suárez-Madrigal, A., Krumholz, M., \& Ramirez-Ruiz, E. 2013, arXiv:1304.2317

Sugimoto, D., Nomoto, K., \& Eriguchi, Y. 1981, PThPS, 70, 115

Suijs, M. P. L., Langer, N., Poelarends, A.-J., et al. 2008, A\&A, 481, L87

Tassoul, M. 1980, ApJS, 43, 469

Thompson, M. J., Christensen-Dalsgaard, J., Miesch, M. S., \& Toomre, J. 2003, ARA\&A, 41, 599

Thompson, M. J., Toomre, J., Anderson, E. R., et al. 1996, Sci, 272, 1300

Tian, C.-L., Deng, L.-C., \& Chan, K.-L. 2009, MNRAS, 398, 1011

Timmes, F. X. 1999, ApJS, 124, 241

Timmes, F. X., \& Swesty, F. D. 2000, ApJS, 126, 501

Torres, G., Andersen, J., \& Giménez, A. 2010, A\&ARv, 18, 67

Townsend, R. H. D., Oksala, M. E., Cohen, D. H., Owocki, S. P., \& ud-Doula, A. 2010, ApJL, 714, L318

Townsley, D. M., \& Bildsten, L. 2004, ApJ, 600, 390

Traxler, A., Garaud, P., \& Stellmach, S. 2011, ApJL, 728, L29

ud-Doula, A., \& Owocki, S. P. 2002, ApJ, 576, 413

Udry, S., \& Santos, N. C. 2007, ARA\&A, 45, 397 
Ulrich, R. K. 1972, ApJ, 172, 165

Unno, W., Osaki, Y., Ando, H., Saio, H., \& Shibahashi, H. 1989, Nonradial Oscillations of Stars (Tokyo: Univ. Tokyo Press)

Ushomirsky, G., Matzner, C. D., Brown, E. F., et al. 1998, ApJ, 497, 253

VandenBerg, D. A., Bergbusch, P. A., \& Dowler, P. D. 2006, ApJS, 162, 375

van Saders, J. L., \& Pinsonneault, M. H. 2012, ApJ, 751, 98

Vink, J. S., de Koter, A., \& Lamers, H. J. G. L. M. 2001, A\&A, 369, 574

von Zeipel, H. 1924, MNRAS, 84, 665

Wachlin, F. C., Miller Bertolami, M. M., \& Althaus, L. G. 2011, A\&A, 533, A139

Weber, E. J., \& Davis, L., Jr. 1967, ApJ, 148, 217

Woo, J.-H., \& Demarque, P. 2001, AJ, 122, 1602

Wood, T. S., Garaud, P., \& Stellmach, S. 2013, ApJ, 768, 157
Woosley, S. E. 1993, ApJ, 405, 273

Woosley, S. E., \& Heger, A. 2006, ApJ, 637, 914

Yang, X. H., Fu, J. N., \& Zha, Q. 2012, AJ, 144, 92

Yoon, S.-C., \& Cantiello, M. 2010, ApJL, 717, L62

Yoon, S.-C., \& Langer, N. 2005, A\&A, 443, 643

Yoon, S.-C., Langer, N., \& Norman, C. 2006, A\&A, 460, 199

Yoon, S.-C., Woosley, S. E., \& Langer, N. 2010, ApJ, 725, 940

York, D. G., Adelman, J., Anderson, J. E., Jr., et al. 2000, AJ, 120, 1579

Young, P. A., Liebst, K., \& Pagano, M. 2012, ApJL, 755, L31

Yurchenko, S. N., Barber, R. J., \& Tennyson, J. 2011, MNRAS, 413, 1828

Zahn, J.-P. 1992, A\&A, 265, 115

Zahn, J.-P., Brun, A. S., \& Mathis, S. 2007, A\&A, 474, 145

Zapolsky, H. S., \& Salpeter, E. E. 1969, ApJ, 158, 809 